\title{
The Second Order Correction to the Ground State Energy of the Dilute Bose Gas
}

\author{
Birger Brietzke ${ }^{1, *} \quad J a n$ Philip Solovej ${ }^{2, *}$ \\ 1. Institute of Applied Mathematics, University of Heidelberg, brietzke@math.uni-heidelberg.de \\ Im Neuenheimer Feld 205, 69120 Heidelberg, Germany \\ 2. Department of Mathematics, University of Copenhagen, solovej@math.ku.dk \\ Universitetsparken 5, 2100 Copenhagen, Denmark
}

\begin{abstract}
We establish the Lee-Huang-Yang formula for the ground state energy of a dilute Bose gas for a broad class of repulsive pair-interactions in 3D as a lower bound. Our result is valid in an appropriate parameter regime of soft potentials and confirms that the Bogolubov approximation captures the right second order correction to the ground state energy.
\end{abstract}

*This work was done in Copenhagen and was partially supported by the Villum Centre of Excellence for the Mathematics of Quantum Theory (QMATH) and the ERC Advanced grant 321029. 


\section{Contents}

1 Introduction $\quad 2$

2 Background Potential and Chemical Potential 6

\begin{tabular}{llr}
3 & Localization & 8 \\
\hline
\end{tabular}

4 Energy in a Single Box $\quad 24$

5 A Priori Bounds on the Non-Quadratic Part of the Hamiltonian and on $n$

6 Estimates on the Large Box

7 Proof of Theorem 2.1 46

A Appendix

\section{Introduction}

Bogolubov's 1947 paper [1] laid the foundation for our present theories of the ground states of dilute Bose gases. His approximate theory was intended to explain the properties of liquid Helium, but it is expected to be most accurate in the opposite regime of a dilute gas of particles (e.g., atoms) interacting pairwise with a repulsive potential $v\left(x_{i}-x_{j}\right) \geq 0$.

The simplest question that can be asked is the correctness of the prediction for the ground state energy. This, of course, can only be exact in a certain limit - the 'weak coupling' limit. In the case of the charged Bose gas that one of the authors studied [13, 14], in which particles interact via Coulomb forces, the appropriate limit is the high density limit. In this setting Bogolubov's prediction, first elucidated in [5], is correct to leading order in the inverse density.

In gases with short range forces, which are the object of study here, the weak coupling limit corresponds to low density instead. The reader is referred to [15] for background information and more details.

Our system consists of $N$ three-dimensional particles in a large box $\Lambda$ of volume $|\Lambda|=L^{3}$. As usual, we are interested in the thermodynamic limit $N \rightarrow \infty,|\Lambda| \rightarrow \infty$ with the ratio $N /|\Lambda| \rightarrow \rho$. The Hamiltonian is

$$
H_{N}=-\nu \sum_{j=1}^{N} \Delta_{j}+\sum_{1 \leq i<j \leq N} v\left(x_{i}-x_{j}\right),
$$


with $\nu=\hbar^{2} / 2 m$, where $m$ is the mass of the particles. From Sect. 2 on we will set $\nu=1$, but we leave it in place in this introduction in order to emphasize that the scattering length of $v$ depends on $\nu$ as well as on $v$. We assume that $v(x) \geq 0$ and that $v$ is spherically symmetric, i.e., $v(x)=v(|x|)$. We could assume that $v$ is a function of sufficiently fast decay at infinity, but in order not to overburden the paper, we assume that $v$ is of finite range, i.e., there is an $R_{0}$ such that $v(x)=0$ for $|x|>R_{0}$. The ground state energy is denoted by $E_{N}$ and

$$
e(\rho)=\lim _{L \rightarrow \infty, N /|\Lambda| \rightarrow \rho} E_{N} /|\Lambda|
$$

denotes the ground state energy density.

The scattering length $a$ is defined by the solution of the equation $-\nu \Delta f(x)+\frac{1}{2} v(x) f(x)=0$ that goes to 1 as $|x| \rightarrow \infty$. Such an $f$ must satisfy $f(x)=1-a /|x|$ for $|x|>R_{0}$. If $v$ is simply a hard core repulsion of radius $r$ then $a=r$.

Bogolubov's formula for the first two terms for $e$ in a small $\rho$ asymptotic expansion is

$$
e(\rho)=4 \pi \nu \rho^{2} a\left(1+\frac{128}{15 \sqrt{\pi}} \sqrt{\rho a^{3}}+o\left(\sqrt{\rho a^{3}}\right)\right)
$$

To be more exact, the leading $4 \pi \nu \rho^{2} a$ term was proposed by Lenz [9]. Bogolubov derived $\frac{1}{2} \rho^{2} \int v(x) \mathrm{d} x$ for the leading term by his method but, realizing that this could not be correct, noticed that $\int v(x) \mathrm{d} x$ is the first Born approximation to $8 \pi \nu a$. The second term, while inherent in Bogolubov's work (see [10, 15]) is credited to Lee, Huang and Yang who actually found it [8] for the hard core gas. Again, Bogolubov's method has $\frac{1}{2} \int v(x) \mathrm{d} x$ in place of $4 \pi \nu a$ in this term as well. It is worth noting that the naive perturbation result, $\frac{1}{2} \int v(x) \mathrm{d} x$, does not depend on $\nu$, which is absurdly incorrect. Other derivations that do not use Bogolubov's setup or the momentum space formulation exist [11, but no rigorous derivation of this second term other than [7], which we discuss below, exists so far.

The first term $4 \pi \nu \rho^{2} a$ was attacked rigorously by Dyson [3] for the hard core case; he proved a variational upper bound of this precise form (up to $o\left(\rho^{2}\right)$ ), as well as a lower bound that, unfortunately, was 14 times too small. He also formulated an inequality that gives a lower bound for the expectation value of $v$ in terms of that of a longer range, softer potential. This inequality has been used in most subsequent rigorous investigations. In particular, it was essential in the paper [16] that finally proved that $4 \pi \nu \rho^{2} a$ is the correct leading term in three-dimensions for any $v \geq 0$ and finite range - including the hard core case.

Our focus here is on the second term. From Bogolubov's perspective it is a correlation effect and in his derivation it presupposes Bose-Einstein condensation (BEC) in the ground 
state. But the fact that his method gives the correct second term in one-dimension [12], despite the fact that there is no BEC in one-dimension, suggests that BEC may not be completely relevant for this problem.

The second term is not merely a perturbation of the first, for it involves new physics. The mean particle spacing is $\rho^{-1 / 3}$, which is much greater than the size of a particle (which we may take to be $a$, not $R$ ). The uncertainty principle tells us that the energy per particle, which is $4 \pi \nu \rho a$, defines a length $\lambda=(\rho a)^{-1 / 2} \gg \rho^{-1 / 3} \gg a$ below which a particle cannot be localized without seriously altering its energy. Thus, it is totally impossible to think of individual particles in the gas; their wave-functions overlap considerably. We can also think of $\lambda$ as the wavelength of the disturbance caused by dropping a particle of size $a \ll \rho^{-1 / 3}$ into the 'sea' of particles. The energy of this very long (on the scale of $\rho^{-1 / 3}$ ) wave, relative to the main term $\nu \rho^{2} a$, can be understood heuristically from the perturbation it causes in the scattering solution resulting in a change of the (two-particle) density, $\rho \rightarrow \rho(1+O(a / \lambda))$. This gives rise to an energy shift $\rho^{2} a \rightarrow \rho^{2} a(1+O(a / \lambda)) \sim \rho^{2} a\left(1+O\left(\sqrt{\rho a^{3}}\right)\right)$.

Until recently it seemed impossible to go beyond the methods of [16] to derive the second term in (3) rigorously. Giuliani and Seiringer [7] have made an important step forward in this quest, however, by considering a situation in which a soft potential $v$ gets fatter and thinner as $\rho \rightarrow 0$ in such a way that $\int v$ is kept constant. In this limit the second Born approximation to the scattering length is of order $v^{2}$, and if $v$ is soft enough one can hope for sufficient accuracy to achieve $4 \pi \nu \rho^{2} a$ in place of Bogolubov's $\frac{1}{2} \rho^{2} \int v(x) \mathrm{d} x$. With the leading term sufficiently under control one can then hope to see the $\sqrt{\rho a^{3}}$ term. In this approach, in which the length scale of the potential is adjustable, the potential has the form

$$
v_{R}(r)=R^{-3} v_{1}(r / R) \quad \text { with } R \rightarrow \infty \text { as } \rho \rightarrow 0
$$

Here $v_{1}$ is a fixed, bounded and sufficiently smooth function with finite (dimensionless) support which we take to be the unit ball such that $v_{R}$ has range $R$. In [7], $v_{1}(r)=a_{0} e^{-r}$.

The interesting question is how $R$ depends on $\rho$ as $\rho \rightarrow 0$. In [7] they take it to be $R \sim$ $\rho^{-1 / 3-7 / 46}$, which implies that each particle 'sees' infinitely many others via the interaction. That is why [7] has "high density" in the title, even though the gas is dilute $\left(a \ll \rho^{-1 / 3}\right)$, and the leading term is still $4 \pi \nu \rho^{2} a$. Nevertheless, this is the first time that the famous $128 \sqrt{\rho a^{3}} / 15 \sqrt{\pi}$ term was seen rigorously as both a lower and an upper bound. Partly relying on the ideas in [13] they achieve a proof of (3). For the upper bound a variational trial state, following [6], was used. Recent progress on the ground state energy and the excitation spectrum for confined gases in a translation invariant setting was made in the series of papers [24, 25, 26, 27]. 
Upper bounds corresponding to (3) were also established in [4], [17] respectively [19], and the latter was extended to higher dimensions in [20].

Our goal is to improve the situation concerning the lower bound a bit. While we still utilize the scaling in (4), our $R$ will also be allowed to be less than $\rho^{-1 / 3}$, which is closer to the physical situation; a particle rarely 'sees' another one now. This will require improving the methodology of [13]. In addition we shall allow for a large class of $v_{1}$.

In the accompanying paper [23] we give a second order lower bound on the ground state energy for the unscaled setting $(R=a)$, which is consistent with (6) but does not capture the sharp constant.

We use the convention

$$
\widehat{f}(p)=\int_{\mathbb{R}^{3}} e^{-i p x} f(x) \mathrm{d} x
$$

for the Fourier transform. Our assumptions on $v_{1}$ and $R$ are the following:

ASSUMPTION 1: We require that $v_{1}$ is non-negative, spherically symmetric, continuous with compact support within the unit ball, in particular, $v_{1} \in L^{1}\left(\mathbb{R}^{3}\right)$, and satisfies $v_{1}(0)>0$.

ASSUMPTION 2: We require, with $\eta<\frac{1}{30}$,

$$
\lim _{\rho \rightarrow 0} \frac{R}{a}\left(\rho a^{3}\right)^{\frac{1}{2}}=0 \quad \text { and } \quad \lim _{\rho \rightarrow 0} R \rho^{1 / 3}\left(\rho a^{3}\right)^{-\eta}=\infty
$$

Our main result is:

THEOREM 1.1. Consider a Bose gas with Hamiltonian (1) where $v$ is replaced by $v_{R}$ given in (4), with $v_{1}$ as in Assumption 1 and $R$ as in Assumption 2. Then, after taking the thermodynamic limit, the energy density, $e(\rho)$, is bounded below by

$$
e(\rho) \geq 4 \pi \nu \rho^{2} a\left(1+\frac{128}{15 \sqrt{\pi}} \sqrt{\rho a^{3}}+o\left(\sqrt{\rho a^{3}}\right)\right) .
$$

Our error terms will depend on the dimensionless quantity $a^{-1} \int v_{1}=a^{-1} \int v_{R}$. Remark on the Born approximation: The 'Born approximation' or 'Born series', is a formula for $a$ as a power series in $v / 8 \pi \nu$.

$$
a=(8 \pi \nu)^{-1} \int_{\mathbb{R}^{3}} v(x) \mathrm{d} x-\sum_{k=2}^{\infty}(-8 \pi \nu)^{-k} \int_{\mathbb{R}^{3}}\left(\mathcal{L}_{v}\right)^{k-1}(v)(x) \mathrm{d} x=: \sum_{k=1}^{\infty} \nu^{-k} a_{k},
$$

where $\mathcal{L}_{v}$ is the operator given by $\mathcal{L}_{v}(g)(x)=v(x) \int_{\mathbb{R}^{3}}|x-y|^{-1} g(y) \mathrm{d} y$. If each term in the series is finite for a given $v$, then, upon replacing $v$ by $v_{R}$ as in (4), the $k^{\text {th }}$ term in the sum, $\nu^{-k} a_{k}$, will be proportional to $R^{1-k}$. Thus, if the series converges for some $R$, it will 
converge for all larger $R$. Convergence will hold for large enough $R$ if $v \in L^{1} \cap L^{\infty}$, but milder conditions suffice.

With the restrictions on $v_{1}$ in Assumption 1 we have that the Born series for $a$ converges and may therefore write

$$
a=a_{1}+a_{2}+O\left(R^{-2}\right)=(8 \pi)^{-1} \widehat{v_{R}}(0)-(4 \pi)^{-1}(2 \pi)^{-3} \int \frac{1}{4} \widehat{v_{R}}(k)^{2}|k|^{-2} \mathrm{~d} k+O\left(R^{-2}\right)
$$

where we have used that $\int \frac{\left|\widehat{v}_{1}(k)\right|^{2}}{|k|^{2}} \mathrm{~d} k=2 \pi^{2} \iint \frac{v_{1}(x) \overline{v_{1}(y)}}{|x-y|} \mathrm{d} x \mathrm{~d} y$. The higher order corrections to $a$ will give contributions to $e$ that are higher order than the term we seek, namely $\rho^{2} a \sqrt{\rho a^{3}}$. For an estimate on the error term in (6) and some additional background we refer to [22].

\section{Background Potential and Chemical Potential}

In order to utilize the technical advantages of second quantization, it is convenient for us to work in Fock space $\mathcal{F}$ (where $N$ takes all values $\geq 0$ ). On Fock space we introduce a Hamiltonian $H_{\rho}$ that depends on a (density) parameter $\rho$. Its action on the $N$-particle sector of Fock space is given by

$$
H_{\rho, N}=\sum_{j=1}^{N}\left(-\Delta_{j}-\rho \int v_{R}(x) \mathrm{d} x\right)+\sum_{1 \leq i<j \leq N} v_{R}\left(x_{i}-x_{j}\right)+\frac{1}{2} \rho^{2}|\Lambda| \int v_{R}(x) \mathrm{d} x
$$

The parameter $\nu=1$ from now on. The box for the particles is $\Lambda=[-L / 2, L / 2] \in \mathbb{R}^{3}$ with Dirichlet boundary conditions. The introduction of the parameter $\rho$ is equivalent to the more common grand canonical approach of adding a term $-\mu N$ with the chemical potential being $\mu=\rho \int v_{R}$. The last term in (9) is simply a constant depending on $\rho$ and we add it for convenience. It is well known [18] that this grand canonical formulation is equivalent to the canonical description (fixed $N$ ) that we started with, but we will not use this fact. In this paper we will focus on the background Hamiltonian $H_{\rho}$ and its thermodynamic ground state energy density

$$
e_{0}(\rho)=\lim _{|\Lambda| \rightarrow \infty}|\Lambda|^{-1} \inf _{\Psi \in \mathcal{F},\|\Psi\|=1}\left\langle\Psi\left|H_{\rho}\right| \Psi\right\rangle .
$$

The ground state energy of $H_{\rho}$ is of course the same as the ground state energy of $H_{\rho, N}$ minimized over $N$. The Fock space may seem irrelevant since $H_{\rho}$ conserves particle number, but will be convenient as we shall be localizing to regions where the particle number is not a priori known. The main goal of our analysis on Fock space is to provide the lower bound on $e_{0}(\rho)$ in Theorem 2.1, which we prove in Section 7. As we will show momentarily, this 
implies our main result, Theorem 1.1 .

THEOREM 2.1 (Ground state of background Hamiltonian).

Let $v_{1}$ satisfy Assumption 1 and let $R$ satisfy Assumption 2. The thermodynamic ground state energy density of $H_{\rho}$ then satisfies the asymptotics, as $\rho \rightarrow 0$,

$$
e_{0}(\rho) \geq 4 \pi \rho^{2}\left(a_{2}+\frac{128}{15 \sqrt{\pi}} a\left(\sqrt{\rho a^{3}}+o\left(\sqrt{\rho a^{3}}\right)\right)\right)
$$

Here $a$ is the scattering length of $v_{R}$ and $a_{2}$ is the second term in the Born series (7) for $a$.

We will now prove the main result Theorem 1.1 from Theorem 2.1.

Proof of Theorem 1.1. By choosing a trial state for $H_{\rho, N}$ corresponding to the ground state for $H_{N}$, we obtain in the thermodynamic limit that

$$
e(\rho) \geq e_{0}(\rho)+\lim _{\substack{|\Lambda| \rightarrow \infty \\ N /|\Lambda| \rightarrow \rho}} \rho \frac{N}{|\Lambda|} \int v_{R}-\frac{1}{2} \rho^{2} \int v_{R}=e_{0}(\rho)+\frac{1}{2} \rho^{2} \int v_{R}
$$

If we recall that $\int v_{R}=8 \pi a_{1}$, we find from Theorem 2.1 that as $\rho \rightarrow 0$

$$
\begin{aligned}
e(\rho) & \geq 4 \pi \rho^{2}\left(a_{2}+\frac{128}{15 \sqrt{\pi}} a\left(\sqrt{\rho a^{3}}+o\left(\sqrt{\rho a^{3}}\right)\right)+4 \pi a_{1} \rho^{2}\right. \\
& =4 \pi \rho^{2} a\left(1+\frac{128}{15 \sqrt{\pi}} \sqrt{\rho a^{3}}+o\left(\sqrt{\rho a^{3}}\right)\right)
\end{aligned}
$$

where we have used that $\frac{a_{1}+a_{2}}{a}=1+O\left(\frac{a^{2}}{R^{2}}\right)=1+o\left(\sqrt{\rho a^{3}}\right)$.

Notation: In our setup the ratio of the scattering length $a$ to $\int v_{R}$ is bounded above and below by constants. In all our error bounds there is therefore no point in distinguishing between $\int v_{R}$ and $a$. We choose to write the estimates in terms of $a$.

The rest of the paper will be devoted to developing tools enabling us to prove Theorem 2.1 in Section 7.

In Section 3 we present a double localization procedure for the kinetic and the potential energy. The double localization should be understood as a method allowing us to estimate the energy on the larger length scale using results obtained on the smaller length scale. In Section 4 we decompose our Hamiltonian into a number of terms by differentiating how it acts on excited particles and particles in the (box) condensate. Then we discuss how the formalism of second quantization can be used to estimate the quadratic part of the Hamiltonian. Effectively this means that we use a Bogoliubov diagonalization. In Section 5.1 
we use a bootstrap argument to first obtain control over the number of particles in a small box, which then leads to a lower bound on the energy. Since the result from the small box is not strong enough to prove our main theorem directly, we transition to the large box in Section 6. On the large box we continue to bootstrap to control the particle number and introduce Theorem 6.5. Lemma 6.6 and Lemma 6.8 to obtain improved control over relevant error terms. Finally, in Section 7 we put the pieces together and give a proof of Theorem 2.1. which, as we already showed, implies our main result, Theorem 1.1 .

\section{Localization}

As usual in the rigorous theory of the ground state energy of the Bose gas we find it necessary to localize the particles into boxes of a certain definite size. This achieves two goals. One is the control of the local fluctuations in particle number and the other is to create a gap in the spectrum of the kinetic operator, which allows us to assert that most particles are in the lowest state of the kinetic energy operator, i.e., they are effectively Bose-condensed on the scale of the box. Alas, this does not allow us to prove Bose-Einstein condensation in the thermodynamic limit, but for the purpose of computing the ground state energy local condensation suffices.

Because there are several length scales, it will turn out to be necessary to localize twice into boxes of two different sizes. The physical length scales of the problem that we are interested in are the following

$$
a \ll R \ll \rho^{-1 / 3} \ll(\rho a)^{-1 / 2}
$$

and these have the following interpretations:

- $a$ is the scattering length of the two-body potential, $v_{R}$.

- $R$ is the range of the potential in case it has compact support and in general it describes the length scale on which the potential vanishes. In our treatment $R$ will be required to be much larger than $a$.

- $\rho^{-1 / 3}$ is the mean particle spacing.

- $(\rho a)^{-1 / 2}$ is the distance determined by the uncertainty principle given that the energy per particle is approximately $\rho a$. In other words if one throws a particle into the gas it makes a splash of size $(\rho a)^{-1 / 2}$. In fact, $(\rho a)^{-1 / 2}$, sometimes called the healing length, is 
the typical distance between the particles in the virtual pairs in the Bogolubov Theory. Momenta of the order of $(\rho a)^{1 / 2}$ are responsible for the second term in (6).

The theorem that we prove includes a bigger range than indicated by (11). If we write $R=\rho^{-1 / 3} Y^{\mu}$ then, as stated in Theorem 1.1, $\mu$ can range in $\left(-\frac{1}{6}, \eta\right)$ with $\eta=\frac{1}{30}$.

The box sizes we are concerned with for localization are $\ell$ and $d \ell$, where $d \ll 1$ in such a way that $\ell \gg(\rho a)^{-1 / 2} \gg d \ell \gg \rho^{-1 / 3}$. Below we will also introduce a small parameter $s>0$ and the length scales $s \ell$ and $d s \ell$. This will give the complete list of length scales 1

$$
a \ll R, \rho^{-1 / 3} \ll d s \ell \ll d \ell \ll(\rho a)^{-1 / 2} \ll s \ell \ll \ell
$$

Although in Theorem 2.1 we also allow $R$ to be much larger than $\rho^{-1 / 3}$, the physically interesting case is, of course, $R \ll \rho^{-1 / 3}$. To be precise, we will in the rest of the paper assume that the following conditions are satisfied.

CONDITION 1: There is a sufficiently small constant $0<\delta<1$ (to be specified in the course of the paper) such that $a, R, s, d, \ell, \rho>0$ satisfy

$$
\begin{aligned}
& a / R<\delta, \quad \rho a^{3}<\delta, \quad \quad \rho^{-\frac{1}{3}}(d s \ell)^{-1}<\delta, \quad s<\delta, \\
& d \ell(\rho a)^{1 / 2}<\delta, \quad R(d s \ell)^{-1}<\delta, \quad(\rho a)^{-1 / 2}(s \ell)^{-1}<\delta .
\end{aligned}
$$

In particular $d<s \delta^{2}$.

More (and stronger) conditions will be added later. As explained, $\delta$ will be chosen in the course of the paper. It will depend on $v_{1}$ and on the integer $M$, which we introduce in (13) below. The integer $M$ will however be chosen at the end and then $\delta$ really depends only on $v_{1}$.

For $u \in \mathbb{R}^{3}$ we introduce the notation $\Gamma_{u}=u+[-1 / 2,1 / 2]^{3}$ for the unit cube centered at $u$. There are three kinds of boxes to be considered. The first is $B(u)=\ell \Gamma_{u}$, which is a cube of side length $\ell$ and center $\ell u$. The second kind is the smaller cube $\widetilde{B}\left(u^{\prime}\right)=(d \ell) \Gamma_{u^{\prime}}$ of side length $d \ell$ and center $d \ell u^{\prime}$. Finally, we have the rectangles $B\left(u, u^{\prime}\right)=B(u) \cap \widetilde{B}\left(u^{\prime}\right)$, which occur when the smaller box is only partially inside the larger box. The second kind is really just a special case of the third kind, so we will not introduce a name for it at this point. Generically, we will let $B$ denote any of these boxes. We denote the side lengths of $B$ by $\lambda_{1} \leq \lambda_{2} \leq \lambda_{3}$.

We now introduce a localization function $0 \leq \chi \in C_{0}^{M}\left(\mathbb{R}^{3}\right)$ where $M$ is an integer to be

\footnotetext{
${ }^{1}$ Note that we allow but not require $R$ to be smaller than $\rho^{-1 / 3}$.
} 
determined in this paper and finally chosen in Lemma 7.1. We let

$$
\zeta(y)=\left\{\begin{array}{ll}
\cos (\pi y), & \text { if }|y| \leq 1 / 2 \\
0, & \text { if }|y| \geq 1 / 2
\end{array} \quad \text { and define } \quad \chi(x)=C_{M}\left(\zeta\left(x_{1}\right) \zeta\left(x_{2}\right) \zeta\left(x_{3}\right)\right)^{M+1}\right.
$$

We choose $C_{M}$ such that $\int \chi^{2}=1$ and note that $\max \chi=\chi(0)=C_{M}$.

It is important not to choose $\chi$ to be infinitely differentiable since the proof of Lemma 3.2 exploits that $\zeta$ is concave on its support. In the following constants may depend on $M$, but we shall mostly omit this fact.

For $u \in \mathbb{R}^{3}$ we write $\chi_{u}(x)=\chi\left(x \ell^{-1}-u\right)$ for the localization function corresponding to the box $B(u)$.

The localization function for the box $B\left(u, u^{\prime}\right)$ is $\chi_{u}(x) \chi_{u^{\prime}}(x / d)$. We introduce the notation

$$
\chi_{B}(x)= \begin{cases}\chi_{u}(x), & \text { if } B=B(u) \\ \chi_{u}(x) \chi_{u^{\prime}}(x / d), & \text { if } B=B\left(u, u^{\prime}\right)\end{cases}
$$

and note that

$$
\int \chi_{u}^{2}(x) \mathrm{d} u=1, \quad \int \chi_{u}^{2}(x) \mathrm{d} x=\ell^{3} \quad \text { and } \quad \int \chi_{B\left(u, u^{\prime}\right)}^{2}(x) \mathrm{d} u^{\prime}=\chi_{B(u)}^{2}(x) .
$$

If $B=B\left(u, u^{\prime}\right)$ is a small box with smallest side length $\lambda_{1}<d \ell$, we have the bound

$$
\max \chi_{B}^{2} \leq C\left[\left(\frac{\lambda_{1}}{\ell}\right)^{M+1}\left(\frac{|B|}{(d \ell)^{3}}\right)^{M+1}\right]^{2} \leq C\left(\frac{\lambda_{1}}{d \ell}\right)^{4(M+1)}
$$

which becomes useful in situations where $\lambda_{1}$ is small.

\subsection{Localization of the Potential}

Corresponding to the interaction potential $v_{R}$, we define two new potentials

$$
W_{\mathrm{b}}(x):=\frac{v_{R}(x)}{(\chi * \chi)(x / \ell)}
$$

and

$$
W_{\mathrm{s}}(x):=\frac{W_{b}(x)}{[(\chi * \chi)(x /(d \ell))]}=\frac{v_{R}(x)}{[(\chi * \chi)(x / \ell)][(\chi * \chi)(x /(d \ell))]} .
$$

Here the subscripts b and s refer to the size of the box (big or small). We will mostly omit this subscript as long as the context is clear. Note that $W_{\mathrm{s}, \mathrm{b}}$ is well defined, since by 
Condition 1 the range $R$ of $v_{R}$ is smaller than the scaled range of $\chi$, which is at least of order $d \ell$. Thus whenever the denominator vanishes, then the numerator is already zero. Since $\chi * \chi$ is a symmetric $C^{2 M}$ function and because $(\chi * \chi)(0)=\int \chi^{2}=1$, we get the estimates

$$
\begin{aligned}
& v_{R}(x) \leq W_{b}(x) \leq\left(1+C\left(\frac{R}{\ell}\right)^{2}\right) v_{R}(x), \\
& v_{R}(x) \leq W_{s}(x) \leq\left(1+C\left(\frac{R}{d \ell}\right)^{2}\right) v_{R}(x) .
\end{aligned}
$$

We introduce localized potentials

$$
w_{B}(x, y):=\chi_{B}(x) W_{\mathrm{b}, \mathrm{s}}(x-y) \chi_{B}(y)=\chi_{B}(x) W(x-y) \chi_{B}(y),
$$

where $b$ is used if the box $B$ is big, i.e., of the form $B(u)$ and $s$ is used if $B$ is small, i.e., of the form $B\left(u, u^{\prime}\right)$. As indicated on the right, we will often omit $\mathrm{b}$ and $\mathrm{s}$. Recall that also the form of the localization functions depends on whether the box is big or small. The potential $w_{B}$ is localized to the box $B$.

Because we do not want to have to consider boxes at the boundary of $\Lambda$ throughout this paper, we introduce $\Lambda^{\prime}:=\Lambda+\left[-\frac{\ell}{2}, \frac{\ell}{2}\right]^{3}$ and $\Lambda^{\prime \prime}:=\Lambda+[-\ell, \ell]^{3}$. Note that $B(u)$ intersects $\Lambda$ exactly if $u \ell \in \Lambda^{\prime}$. Replacing the last term in (9) by $\frac{1}{2} \rho^{2}\left|\Lambda^{\prime \prime}\right| \int v_{R}(x) \mathrm{d} x$ does not change the ground state energy of $H_{\rho}$ in the thermodynamic limit. We may therefore use the following localization for the potential energy.

PROPOSITION 3.1 (Potential localization). For all $x_{1}, \ldots, x_{N} \in \Lambda$ we have

$$
\begin{aligned}
& -\sum_{j=1}^{N} \rho \int v_{R}\left(x_{j}-y\right) \mathrm{d} y+\sum_{1 \leq i<j \leq N} v_{R}\left(x_{i}-x_{j}\right)+\frac{1}{2} \rho^{2}\left|\Lambda^{\prime \prime}\right| \int v_{R}(x) \mathrm{d} x \\
= & \int_{\mathbb{R}^{3}}\left(-\sum_{j=1}^{N} \rho \int \omega_{B(u)}\left(x_{j}, y\right) \mathrm{d} y+\sum_{1 \leq i<j \leq N} \omega_{B(u)}\left(x_{i}, x_{j}\right)+\frac{1}{2} \rho^{2} \iint_{\mathbb{R}^{3} \times \Lambda^{\prime \prime}} \omega_{B(u)}(x, y) \mathrm{d} x \mathrm{~d} y\right) \mathrm{d} u \\
\geq & \int_{\ell^{-1} \Lambda^{\prime}}\left(-\sum_{j=1}^{N} \rho \int \omega_{B(u)}\left(x_{j}, y\right) \mathrm{d} y+\sum_{1 \leq i<j \leq N} \omega_{B(u)}\left(x_{i}, x_{j}\right)+\frac{1}{2} \rho^{2} \iint \omega_{B(u)}(x, y) \mathrm{d} x \mathrm{~d} y\right) \mathrm{d} u,
\end{aligned}
$$

where $\Lambda^{\prime}:=\Lambda+\left[-\frac{\ell}{2}, \frac{\ell}{2}\right]^{3}$ and $\Lambda^{\prime \prime}:=\Lambda+[-\ell, \ell]^{3}$. Moreover, for all $u \in \mathbb{R}^{3}$,

$$
-\sum_{j=1}^{N} \rho \int \omega_{B(u)}\left(x_{j}, y\right) \mathrm{d} y+\sum_{1 \leq i<j \leq N} \omega_{B(u)}\left(x_{i}, x_{j}\right)+\frac{1}{2} \rho^{2} \iint \omega_{B(u)}(x, y) \mathrm{d} x \mathrm{~d} y
$$


$=\int_{\mathbb{R}^{3}}\left(-\sum_{j=1}^{N} \rho \int \omega_{B\left(u, u^{\prime}\right)}\left(x_{j}, y\right) \mathrm{d} y+\sum_{1 \leq i<j \leq N} \omega_{B\left(u, u^{\prime}\right)}\left(x_{i}, x_{j}\right)+\frac{1}{2} \rho^{2} \iint \omega_{B\left(u, u^{\prime}\right)}(x, y) \mathrm{d} x \mathrm{~d} y\right) \mathrm{d} u^{\prime}$.

Proof. This follows from the identity $(\chi * \chi)(x-y)=\int \chi(x-u) \chi(y-u) \mathrm{d} u$.

The background self-energy appears so frequently that we shall denote it $\rho^{2}|B|^{2} \mathcal{U}_{B}$, i.e., we introduce the symbol

$$
\mathcal{U}_{B}=\frac{1}{2}|B|^{-2} \iint w_{B}(x, y) \mathrm{d} x \mathrm{~d} y
$$

On the large box $B(u)$ we use that $\chi$ and $\chi * \chi$ are even such that for all $u \in \mathbb{R}^{3}$

$$
\begin{aligned}
\mathcal{U}_{B} & =\frac{1}{2} \ell^{-6} \iint_{\mathbb{R}^{6}} w_{B(u)}(x, y) \mathrm{d} x \mathrm{~d} y=\frac{1}{2} \ell^{-6} \iint_{\mathbb{R}^{6}} \chi\left(-\frac{x}{\ell}\right) \chi\left(\frac{y}{\ell}\right) W(x-y) \mathrm{d} x \mathrm{~d} y \\
& =\frac{1}{2} \iint_{\mathbb{R}^{6}} \chi(-x-y) \chi(y) W(\ell x) \mathrm{d} x \mathrm{~d} y=\frac{1}{2} \int_{\mathbb{R}^{3}} v_{R}(\ell x) \mathrm{d} x=\frac{4 \pi a_{1}}{\ell^{3}} .
\end{aligned}
$$

In a small (possibly rectangular) box $B, \mathcal{U}_{B}$ may be significantly different. It will be important to know the following facts.

LEMMA 3.2. If $B$ is either a large or a small box (side lengths $\lambda_{1} \leq \lambda_{2} \leq \lambda_{3}$ ), there is a constant $C$ that depends only on $M$ used in the definition of $\chi$ and on the potential $v_{1}$ in (4) such that

$$
\begin{gathered}
\max _{x} \int w_{B}(x, y) \mathrm{d} y \leq \frac{1}{2} C|B|^{-1} \iint w_{B}(x, y) \mathrm{d} y \mathrm{~d} x=C|B| \mathcal{U}_{B} \\
C^{-1} \frac{a}{|B| R^{3}} \max \chi_{B}^{2} \prod_{j=1}^{3} \min \left\{\lambda_{j}, R\right\} \leq \mathcal{U}_{B} \leq C \frac{a}{R^{3}} \max \chi_{B}^{2} \\
\mathcal{U}_{B} \leq C \frac{a}{|B|} \max \chi_{B}^{2} .
\end{gathered}
$$

(The scattering length a, which obviously is bounded above and below by constants depending only on $v_{1}$, has been included in this inequality for dimensional reasons.)

Proof. The difficult case is a (possibly) rectangular box $B=B\left(u, u^{\prime}\right)$, for the large cubic boxes $B=B(u)$ the argument is the same just simpler. Recall that for a small box we have $w_{B}(x, y)=\chi_{B}(x) W_{\mathrm{s}}(x-y) \chi_{B}(y)$ and $\chi_{B}(x)=\chi_{1}\left(x_{1}\right) \chi_{2}\left(x_{2}\right) \chi_{3}\left(x_{3}\right)$, where

$$
\chi_{i}\left(x_{i}\right)=C_{M}^{2 / 3} \zeta\left(\left|\left(x_{i} / \ell\right)-u_{i}\right|\right)^{M+1} \zeta\left(\left|\left(x_{i} /(d \ell)\right)-u_{i}^{\prime}\right|\right)^{M+1}
$$


for $i=1,2,3$. The function $\chi_{i}$ is supported on an interval $I_{i}$ of length $\lambda_{i}$ corresponding to a side length of the box. We have $0<\lambda_{i} \leq d \ell$. Let $I_{i}^{\prime}$ denote the middle third of this interval. Since $\zeta$ is positive and concave on its support, it is a straightforward exercise to check that

$$
\inf _{x_{i} \in I_{i}^{\prime}} \chi_{i}\left(x_{i}\right) \geq c \max _{x_{i} \in I_{i}} \chi_{i}\left(x_{i}\right)
$$

for $i=1,2,3$, where $c>0$ depends only on $M$. It is important here that $\chi$ is not infinitely differentiable.

By (4), 20) and the fact that $v_{1}$ is continuous, has compact support and $v_{1}(0)>0$ there exist constants $C_{1}, C_{2}>0$ (depending only on $v_{1}$ ) such that

$$
C_{1} \prod_{i=1}^{3} \mathbb{1}_{\left[-C_{1} R, C_{1} R\right]}\left(x_{i}\right) \leq a^{-1} R^{3} W_{\mathrm{s}}(x) \leq C_{2} \prod_{i=1}^{3} \mathbb{1}_{\left[-C_{2} R, C_{2} R\right]}\left(x_{i}\right),
$$

where $\mathbb{1}_{I}$ is the characteristic function of the interval $I$. To prove the inequality (24), it is therefore enough to prove the 1-dimensional versions:

$\max _{x_{i} \in \mathbb{R}} \int \chi_{i}\left(x_{i}\right) \mathbb{1}_{\left[-C_{2} R, C_{2} R\right]}\left(x_{i}-y_{i}\right) \chi_{i}\left(y_{i}\right) \mathrm{d} y_{i} \leq C \lambda_{i}^{-1} \iint \chi_{i}\left(x_{i}\right) \mathbb{1}_{\left[-C_{1} R, C_{1} R\right]}\left(x_{i}-y_{i}\right) \chi_{i}\left(y_{i}\right) \mathrm{d} y_{i} \mathrm{~d} x_{i}$,

for $i=1,2,3$, where $C$ is allowed to depend only on $v_{1}$ and $M$. In view of (27) this follows from

$$
\max _{x_{i} \in \mathbb{R}} \int_{I_{i}} \mathbb{1}_{\left[-C_{2} R, C_{2} R\right]}\left(x_{i}-y_{i}\right) \mathrm{d} y_{i} \leq C \lambda_{i}^{-1} \iint_{I_{i}^{\prime} \times I_{i}^{\prime}} \mathbb{1}_{\left[-C_{1} R, C_{1} R\right]}\left(x_{i}-y_{i}\right) \mathrm{d} y_{i} \mathrm{~d} x_{i} .
$$

This is obvious since both sides can be estimated above and below by constants times $\min \left\{\lambda_{i}, R\right\}$.

The lower bound in 25 is proved in a similar fashion. The upper bound in (25) follows from $v_{R}(x) \leq C \frac{a}{R^{3}}$.

For the bound in 26 we note that $\mathcal{U}_{B} \leq C|B|^{-1} \int_{\mathbb{R}^{3}} v_{R}(x) \max \chi_{B}^{2} \mathrm{~d} x \leq C \frac{a}{|B|} \max \chi_{B}^{2}$, since $\omega_{B}(x, y) \leq C v_{R}(x-y) \max \chi_{B}^{2}$ and that by Condition 1 we have $\int v_{R}(x) \mathrm{d} x \leq C a$.

\subsection{Localization of the Kinetic Energy}

Let $\theta$ be the characteristic function of the cubic box $[-1 / 2,1 / 2]^{3}$. For $u \in \mathbb{R}^{3}$ we denote the corresponding characteristic function of the box $B(u)$ by $\theta_{u}(x)=\theta\left(x \ell^{-1}-u\right)$. We shall also use the localization function $\chi_{u}(x)=\chi\left(x \ell^{-1}-u\right)$ introduced on page 3 .

We define the operator $Q_{u}$ to be the orthogonal projection on $L^{2}\left(\mathbb{R}^{3}\right)$ defined by

$$
Q_{u} f=\theta_{u} f-\ell^{-3}\left\langle\theta_{u} \mid f\right\rangle \theta_{u}
$$


In other words $Q_{u} f$ is a function in $L^{2}\left(\mathbb{R}^{3}\right)$ that is zero outside the box $B(u)$ and is orthogonal to the constant functions in the box.

LEMMA 3.3 (Abstract kinetic energy localization). Let $\mathcal{K}: \mathbb{R}^{3} \rightarrow[0, \infty)$ be a symmetric, continuous function, which is bounded by a polynomial of degree at most $2 M$, where $M$ is the integer introduced in 13 . We use it to define an operator on $L^{2}\left(\mathbb{R}^{3}\right)$ by

$$
T=\int_{\mathbb{R}^{3}} Q_{u} \chi_{u} \mathcal{K}(-i \ell \nabla) \chi_{u} Q_{u} \mathrm{~d} u
$$

where $\chi_{u}$ is considered here as a multiplication operator in configuration space. This $T$ is translation invariant, i.e., a multiplication operator in Fourier space $T=F(-i \ell \nabla)$, with

$$
F(p)=(2 \pi)^{-3} \mathcal{K} *|\widehat{\chi}|^{2}(p)-2(2 \pi)^{-3} \widehat{\theta}(p) \widehat{\chi} *(\mathcal{K} \widehat{\chi})(p)+(2 \pi)^{-3}\left(\int \mathcal{K}|\widehat{\chi}|^{2}\right) \widehat{\theta}(p)^{2}
$$

In particular, we have $F(0)=0, F \geq 0$ and $\nabla F(0)=0$.

Proof. By a simple scaling it is enough to consider $\ell=1$. This is a straightforward calculation. Note that $Q_{u}$ has the integral kernel $\theta_{u}(y)[\delta(y-x)-\mathbb{1}] \theta_{u}(x)$. If we denote by $\check{\mathcal{K}}$ the inverse Fourier transform of $\mathcal{K}$ in the sense of a tempered distribution, then the integral kernel of the operator $Q_{u} \chi_{u} \mathcal{K}(-i \nabla) \chi_{u} Q_{u}$ is given by

$$
\begin{aligned}
& \chi_{u}(x) \check{\mathcal{K}}(x-y) \chi_{u}(y)-\chi_{u}(x)\left[\check{\mathcal{K}} * \chi_{u}\right](x) \theta_{u}(y) \\
& -\theta_{u}(x)\left[\check{\mathcal{K}} * \chi_{u}\right](y) \chi_{u}(y)+\theta_{u}(x)\left\langle\chi_{u} \mid \mathcal{K}(-i \nabla) \chi_{u}\right\rangle \theta_{u}(y) .
\end{aligned}
$$

Thus the integral kernel of $\int Q_{u} \chi_{u} \mathcal{K}(-i \nabla) \chi_{u} Q_{u} \mathrm{~d} u$ is given by

$$
([\chi * \chi] \check{\mathcal{K}})(x-y)-2(\chi[\check{\mathcal{K}} * \chi]) * \theta(x-y)+(2 \pi)^{-3}\left(\int \mathcal{K}(p) \widehat{\chi}(p)^{2} \mathrm{~d} p\right) \theta * \theta(x-y)
$$

where we used that $\int \mathcal{K}(p) \widehat{\chi}(p)^{2} \mathrm{~d} p$ is finite by the choice of $\mathcal{K}$. We arrive at the expression for $F$ by calculating the inverse Fourier transform. The fact that $F(0)=0$ follows since $\widehat{\theta}(0)=\int \theta=1$ and

$$
(2 \pi)^{3} F(0)=2\left(\int K \widehat{\chi}^{2}\right)(1-\widehat{\theta}(0))^{2}=0 .
$$

That $F \geq 0$ is a direct consequence of $(29)$ since $\mathcal{K}$ is positive. Because $F$ is differentiable it follows that $\nabla F(0)=0$. 
With $\ell=1$ this lemma is similar to the generalized IMS localization formula

$$
\int_{\mathbb{R}^{3}} \chi_{u} \mathcal{K}(-i \nabla) \chi_{u} \mathrm{~d} u=(2 \pi)^{-3} \mathcal{K} *|\widehat{\chi}|^{2}
$$

where $\mathcal{K}(p)=p^{2}$ gives the standard IMS formula since $(2 \pi)^{-3} \mathcal{K} *|\widehat{\chi}|^{2}=p^{2}+\int|\nabla \chi|^{2}$.

COROLLARY 3.4. With the same notation as above we have that

$$
\int_{\mathbb{R}^{3}} Q_{u} \mathrm{~d} u=1-\widehat{\theta}(-i \ell \nabla)^{2}
$$

i.e., the operator $\int_{\mathbb{R}^{3}} Q_{u} \mathrm{~d} u$ is the multiplication operator in Fourier space given by $1-\widehat{\theta}(\ell p)^{2}$.

Proof. Simply take $\mathcal{K}=1$ and $\chi=\theta$ in the above lemma.

We will use Lemma 3.3 for the function $\mathcal{K}(p)=\left(\left(|p|-s^{-1}\right)_{+}\right)^{2}$, where $s>0$ is the parameter introduced in Condition 1. Here $u_{+}=\max \{u, 0\}$ denotes the positive part of $u$ and we will henceforth write $u_{+}^{2}$ instead of $\left(u_{+}\right)^{2}$. Note that $|-i \nabla|=\sqrt{-\Delta}$.

LEMMA 3.5. There exist constants $C>0$ and $s^{*}>0$ (depending on the integer $M$ in the definition (13) of $\chi$ ) such that for $0<s \leq s^{*}$ and any $\ell>0$ we have the inequality for all $\varphi \in H^{1}\left(\mathbb{R}^{3}\right)$

$$
\left\langle\varphi, F_{s}(\sqrt{-\Delta}) \varphi\right\rangle \geq \int\left\langle\varphi, Q_{u} \chi_{u}\left[\sqrt{-\Delta}-(s \ell)^{-1}\right]_{+}^{2} \chi_{u} Q_{u} \varphi\right\rangle \mathrm{d} u
$$

where

$$
F_{s}(|p|)= \begin{cases}\left(|p|-\frac{1}{2}(s \ell)^{-1}\right)^{2}, & \text { if }|p| \geq \frac{5}{6}(s \ell)^{-1} \\ C s^{M-2} p^{2}, & \text { if }|p|<\frac{5}{6}(s \ell)^{-1}\end{cases}
$$

(assuming $M \geq 2$ )

Proof. We may again by a simple scaling argument assume $\ell=1$. Since we defined $\chi$ in (13) as a $C_{0}^{M}$ function, we have for $n \leq 2 M$ and $C$ only depending on $M$ that

$$
\int_{|q|>s^{-1}}|q|^{n} \widehat{\chi}^{2}(q) \mathrm{d} q \leq s^{2 M-n} \int_{|q|>s^{-1}}|q|^{2 M} \widehat{\chi}^{2}(q) \mathrm{d} q \leq C s^{2 M-n} .
$$

We use 29 and 30 with $\mathcal{K}(p)=\left[|p|-s^{-1}\right]_{+}^{2}$. For the first term in 30 we find

$$
\begin{aligned}
(2 \pi)^{-3} \mathcal{K} * \widehat{\chi}^{2}(p) & \leq(2 \pi)^{-3} \int\left(|p-q|-s^{-1}\right)^{2} \widehat{\chi}^{2}(q) \mathrm{d} q \\
& \leq(2 \pi)^{-3} \int\left(p^{2}-2 p q+q^{2}-2 s^{-1}(|p|-|q|)+s^{-2}\right) \widehat{\chi}^{2}(q) \mathrm{d} q
\end{aligned}
$$




$$
=\left(|p|-s^{-1}\right)^{2}+C+C s^{-1}
$$

where we have used $(2 \pi)^{-3} \int q^{2} \widehat{\chi}(q)^{2} \mathrm{~d} q=\int|\nabla \chi|^{2}$ and that $\chi^{2}$ is even.

If $|p| \geq \frac{5}{6} s^{-1}$ we thus find

$$
(2 \pi)^{-3} \mathcal{K} * \widehat{\chi}^{2}(p) \leq\left(|p|-\frac{1}{2} s^{-1}\right)^{2}-\frac{1}{12} s^{-2}+C+C s^{-1}
$$

For the second term in 30 we find since $\widehat{\theta} \leq 1$ that

$$
|\widehat{\theta}(p) \widehat{\chi} *(\mathcal{K} \widehat{\chi})(p)| \leq\|\widehat{\chi}\|_{2}|| \mathcal{K} \widehat{\chi} \|_{2} \leq C\left(\int_{|q| \geq s^{-1}}|q|^{4}|\widehat{\chi}(q)|^{2} \mathrm{~d} q\right)^{1 / 2} \leq C s^{M-2}
$$

For the third term in 30 we have similarly

$$
|\widehat{\theta}(p)|^{2} \int \mathcal{K}|\widehat{\chi}|^{2} \leq \int_{|q| \geq s^{-1}}|q|^{2} \widehat{\chi}(q)^{2} \mathrm{~d} q \leq C s^{2 M-2}
$$

For $|p| \geq \frac{5}{6} s^{-1}$ we therefore have that the function $F$ in 30 satisfies

$$
F(p) \leq\left(|p|-\frac{1}{2} s^{-1}\right)^{2}-\frac{1}{12} s^{-2}+C s^{-1}+C s^{M-2}+C s^{2 M-2} .
$$

With $s^{*}$ sufficiently small we arrive at the first line in (33).

We turn to the proof of the second line in 33 . We know that $F(0)=\nabla F(0)=0$. The lemma follows from Taylor's formula if we can show that for $|p|<\frac{5}{6} s^{-1}$, we have

$$
\left|\partial_{i} \partial_{j} F(p)\right| \leq C s^{M-2}
$$

It is straightforward to see that all second derivatives of $\mathcal{K}(p)=\left[|p|-s^{-1}\right]_{+}^{2}$ are bounded independently of $s$. For the first term in 30 we thus find for $|p|<\frac{5}{6} s^{-1}$

$$
\begin{aligned}
\left|\partial_{i} \partial_{j}\left(\mathcal{K} * \widehat{\chi}^{2}\right)(p)\right| & =\left|\left(\partial_{i} \partial_{j} \mathcal{K}\right) * \widehat{\chi}^{2}(p)\right| \leq C \int_{|p-q|>s^{-1}} \widehat{\chi}(q)^{2} \mathrm{~d} q \\
& \leq C \int_{|q|>(6 s)^{-1}} \widehat{\chi}(q)^{2} \mathrm{~d} q \leq C s^{2 M} .
\end{aligned}
$$

For the second and third term in 30 we use the fact that for all $i, j=1,2,3$ the numbers

$$
\|\widehat{\theta}\|_{\infty},\left\|\partial_{i} \widehat{\theta}\right\|_{\infty},\left\|\partial_{i} \partial_{j} \widehat{\theta}\right\|_{\infty}, \int|\widehat{\chi}|^{2}, \quad \int\left|\partial_{i} \widehat{\chi}\right|^{2}, \quad \int\left|\partial_{i} \partial_{j} \widehat{\chi}\right|^{2}
$$


are bounded by a constant. The same estimates that led to (36) and (37) then imply (38).

COROLLARY 3.6. If $M \geq 3$ there exist constants $b>0$ and $s^{\prime}>0$ (depending on the integer $M$ in the definition (13) of $\chi$ ) such that for $0<s \leq s^{\prime}$ and any $\ell>0$ we have for all $\varphi \in H_{0}^{1}(\Lambda)$

$$
\langle\varphi,-\Delta \varphi\rangle \geq \int_{\mathbb{R}^{3}}\left\langle\varphi, Q_{u}\left\{\chi_{u}\left[\sqrt{-\Delta}-\frac{1}{2}(s \ell)^{-1}\right]_{+}^{2} \chi_{u}+b \ell^{-2}\right\} Q_{u} \varphi\right\rangle \mathrm{d} u
$$

Proof. We again consider $\ell=1$. Note that by Corollary 3.4 and a Taylor expansion at $p=0$ we have

$$
\int Q_{u} \mathrm{~d} u \leq \beta^{-1} \frac{-\Delta}{-\Delta+\beta}
$$

for a universal constant $0<\beta<1$. We use the previous lemma with $s$ replaced by $2 s$. We then find that

$$
\int_{\mathbb{R}^{3}} Q_{u} \chi_{u}\left[\sqrt{-\Delta}-\frac{1}{2} s^{-1}\right]_{+}^{2} \chi_{u} Q_{u} \mathrm{~d} u+b \int_{\mathbb{R}^{3}} Q_{u} \mathrm{~d} u \leq F_{2 s}(\sqrt{-\Delta})+b \beta^{-1} \frac{-\Delta}{-\Delta+\beta} .
$$

For $|p|<(5 / 12) s^{-1}$ Lemma 3.5 gives

$$
F_{2 s}(p)+b \beta^{-1} \frac{p^{2}}{p^{2}+\beta} \leq C s p^{2}+b \beta^{-1} \frac{p^{2}}{p^{2}+\beta} \leq\left(C s+b \beta^{-2}\right) p^{2} \leq p^{2}
$$

for $s$ sufficiently small. For $|p| \geq(5 / 12) s^{-1}$ we find from Lemma 3.5 that

$$
F_{2 s}(p)+b \beta^{-1} \frac{p^{2}}{p^{2}+\beta} \leq\left(|p|-\frac{1}{4} s^{-1}\right)^{2}+b \beta^{-1} \frac{p^{2}}{p^{2}+\beta} \leq p^{2}-\frac{5}{24} s^{-2}+\frac{1}{16} s^{-2}+b \beta^{-1} \leq p^{2}
$$

for $s$ sufficiently small.

This corollary will allow us to localize the kinetic energy to boxes. What will be left as the kinetic energy in the box $B(u)$ centered at $\ell u$, is the operator

$$
\mathcal{T}_{u}=Q_{u}\left\{\chi_{u}\left[\sqrt{-\Delta}-\frac{1}{2}(s \ell)^{-1}\right]_{+}^{2} \chi_{u}+b \ell^{-2}\right\} Q_{u}
$$

Note that $\mathcal{T}_{u}$ vanishes on constant functions. The last term in $\mathcal{T}_{u}$ will control the gap in the kinetic energy, i.e., on functions orthogonal to constants in the box, $\mathcal{T}_{u}$ is bounded below by at least $b \ell^{-2}$.

In 23] we modify the localization of the kinetic energy, thereby avoiding an error term originating from A.15, which would not be compatible with the LHY-order in the unscaled setting of [23]. We expect that a corresponding modification in the present paper would 
allow us to state Assumption 2 with $\eta=\frac{1}{30}$ instead of $\eta<\frac{1}{30}$.

As explained above, we need to localize even further to smaller boxes whose size is a factor $d \ll 1$ smaller than the larger box. In these smaller boxes we also need a term in the kinetic energy that gives a gap. Unfortunately, the above expression (40) for the kinetic energy does not immediately allow for such further localization. For this reason we must introduce a more complicated kinetic energy in the larger box.

We will use

$$
\begin{aligned}
\widehat{\mathcal{T}}_{u}= & \varepsilon_{T}(d \ell)^{-2} \frac{-\Delta_{u}^{\mathcal{N}}}{-\Delta_{u}^{\mathcal{N}}+(d \ell)^{-2}}+b \ell^{-2} Q_{u} \\
& +Q_{u} \chi_{u}\left\{\left(1-\varepsilon_{T}\right)\left[\sqrt{-\Delta}-\frac{1}{2}(s \ell)^{-1}\right]_{+}^{2}+\varepsilon_{T}\left[\sqrt{-\Delta}-\frac{1}{2}(d s \ell)^{-1}\right]_{+}^{2}\right\} \chi_{u} Q_{u},
\end{aligned}
$$

where $0<\varepsilon_{T}<1$ is a parameter. The operator $\Delta_{u}^{\mathcal{N}}$ is the Neumann Laplacian on the box $B(u)$. As usual $\Delta$ is the Laplacian on $\mathbb{R}^{3}$. Let us be clear about the action of $\Delta_{u}^{\mathcal{N}}$ as an operator on $L^{2}\left(\mathbb{R}^{3}\right)$. It is the operator associated with the quadratic form

$$
\left(f,-\Delta_{u}^{\mathcal{N}} f\right)=\int_{B(u)}|\nabla f(x)|^{2} \mathrm{~d} x,
$$

which is defined for all functions $f \in L^{2}\left(\mathbb{R}^{3}\right)$ whose restriction to the cube is an $H^{1}$ function, i.e., functions for which the above integral is finite. Note that by the operator $\left(-\Delta_{u}^{\mathcal{N}}+\right.$ $\left.(d \ell)^{-2}\right)^{-1}$ we mean the inverse of $-\Delta_{u}^{\mathcal{N}}+(d \ell)^{-2}$ on the space $L^{2}(B(u))$ extended to all of $L^{2}\left(\mathbb{R}^{3}\right)$ by letting it be 0 on the orthogonal complement, i.e., on functions that live outside $B(u)$.

Note that if $\varepsilon_{T}=0$ then $\widehat{\mathcal{T}}_{u}$ equals $\mathcal{T}_{u}$. For the kinetic energy $\widehat{\mathcal{T}}_{u}$ we have a result similar to Corollary 3.6. For the following theorem we note that on the domain $H_{0}^{1}(\Lambda)$ we have

$$
\int-\Delta_{u}^{\mathcal{N}} \mathrm{d} u=-\Delta_{D}
$$

in the sense of quadratic forms.

LEMMA 3.7 (Large-box kinetic energy localization). If $M \geq 5$, and $b, d, s, \varepsilon_{T}>0$ are smaller than some universal constant, then

$$
\int_{\mathbb{R}^{3}} \widehat{\mathcal{T}}_{u} \mathrm{~d} u \leq-\Delta
$$


Proof. As usual we set $\ell=1$. The first step in the proof is to show that for all $d>0$

$$
\int_{\mathbb{R}^{3}} \frac{-\Delta_{u}^{\mathcal{N}}}{-\Delta_{u}^{\mathcal{N}}+d^{-2}} \mathrm{~d} u \leq \frac{-\Delta}{-\Delta+d^{-2}}
$$

To show this, we recall that in the sense of quadratic forms $\sum_{u \in \mathbb{Z}^{3}}-\Delta_{u}^{\mathcal{N}} \leq-\Delta$. Thus

$$
\left(-\Delta+d^{-2}\right)^{-1} \leq\left(\sum_{u \in \mathbb{Z}^{3}}-\Delta_{u}^{\mathcal{N}}+d^{-2}\right)^{-1}=\sum_{u \in \mathbb{Z}^{3}}\left(-\Delta_{u}^{\mathcal{N}}+d^{-2}\right)^{-1}
$$

The last equality looks odd, but it is just the identity $\left(\bigoplus_{u} A_{u}\right)^{-1}=\bigoplus_{u} A_{u}^{-1}$ applied to the operators $A_{u}=-\Delta_{u}^{\mathcal{N}}+d^{-2} \mathbb{1}_{L^{2}(B(u))}$ acting on $L^{2}(B(u))$ recalling that $L^{2}\left(\mathbb{R}^{3}\right)=$ $\bigoplus_{u \in \mathbb{Z}^{3}} L^{2}(B(u))$.

Since $1-d^{-2}\left(-\Delta+d^{-2}\right)^{-1}=-\Delta\left(-\Delta+d^{-2}\right)^{-1}$, it follows from 45 that

$$
\sum_{u \in \mathbb{Z}^{3}} \frac{-\Delta_{u}^{\mathcal{N}}}{-\Delta_{u}^{\mathcal{N}}+d^{-2}} \leq \frac{-\Delta}{-\Delta+d^{-2}}
$$

This will also hold if we replace the sum over $\mathbb{Z}^{3}$ by a sum over $v+\mathbb{Z}^{3}$ for any $v \in[0,1]^{3}$. An integration over $v \in[0,1]^{3}$ gives 44 ).

The second step is to observe that from Lemma 3.5, e.g., with $M \geq 5$ we find that

$$
\begin{gathered}
\int Q_{u} \chi_{u}\left[\left(1-\varepsilon_{T}\right)\left[\sqrt{-\Delta}-\frac{1}{2} s^{-1}\right]_{+}^{2}+\varepsilon_{T}\left[\sqrt{-\Delta}-\frac{1}{2}(d s)^{-1}\right]_{+}^{2}\right] \chi_{u} Q_{u} \mathrm{~d} u \\
\leq\left(1-\varepsilon_{T}\right)\left[\sqrt{-\Delta}-\frac{1}{4} s^{-1}\right]_{+}^{2}+\varepsilon_{T}\left[\sqrt{-\Delta}-\frac{1}{4}(d s)^{-1}\right]_{+}^{2}+C s \frac{-\Delta}{-\Delta+\beta}
\end{gathered}
$$

for some universal constant $C$ and where $\beta$ is the same constant as in (39). The proof is completed using 39 and observing that if $s, b, d, \varepsilon_{T}$ are all smaller than some universal constant, then, for all $p \in \mathbb{R}^{3}$,

$$
\left(b \beta^{-1}+C s\right) \frac{p^{2}}{p^{2}+\beta}+\varepsilon_{T} d^{-2} \frac{p^{2}}{p^{2}+d^{-2}}+\left(1-\varepsilon_{T}\right)\left[|p|-\frac{1}{4} s^{-1}\right]_{+}^{2}+\varepsilon_{T}\left[|p|-\frac{1}{4}(d s)^{-1}\right]_{+}^{2} \leq p^{2} .
$$

We now discuss the further localization into smaller boxes of relative size $d \ll 1$. As in the previous subsection we index these boxes by a parameter $u^{\prime} \in \mathbb{R}^{3}$. The small box is $\widetilde{B}\left(u^{\prime}\right)=d \ell \Gamma_{u^{\prime}}=d \ell u^{\prime}+[-d \ell / 2, d \ell / 2]^{3}$, whose center is at $d \ell u^{\prime}$. We denote the corresponding 
characteristic function and localization function by

$$
\widetilde{\theta}_{u^{\prime}}(x)=\theta\left((x / d \ell)-u^{\prime}\right), \quad \widetilde{\chi}_{u^{\prime}}(x)=\chi\left((x / d \ell)-u^{\prime}\right) .
$$

The corresponding orthogonal projection onto functions orthogonal to constants in $L^{2}\left(\widetilde{B}\left(u^{\prime}\right)\right)$ is $\widetilde{Q}_{u^{\prime}}$ given by

$$
\widetilde{Q}_{u^{\prime}} f=\widetilde{\theta}_{u^{\prime}} f-(d \ell)^{-3}\left\langle\widetilde{\theta}_{u^{\prime}} \mid f\right\rangle \widetilde{\theta}_{u^{\prime}}
$$

When localizing in to the smaller boxes, we are forced to consider the situation of overlap between the large boxes and small boxes, i.e., $B\left(u, u^{\prime}\right)=B(u) \cap \widetilde{B}\left(u^{\prime}\right)$. The corresponding characteristic function is $\theta_{u} \widetilde{\theta}_{u^{\prime}}$, the corresponding localization function is $\chi_{u} \widetilde{\chi}_{u^{\prime}}$, and the corresponding orthogonal projection is

$$
Q_{u u^{\prime}} f=\theta_{u} \widetilde{\theta}_{u^{\prime}} f-\left|B\left(u, u^{\prime}\right)\right|^{-1}\left\langle\theta_{u} \widetilde{\theta}_{u^{\prime}} \mid f\right\rangle \theta_{u} \widetilde{\theta}_{u^{\prime}}
$$

Our first result is that when we localize the large box kinetic energy $\widehat{\mathcal{T}}_{u}$ into smaller boxes we will get a gap in the localized energy spectrum. This is a consequence of the next result.

LEMMA 3.8. With $Q_{u u^{\prime}}$ as defined above we have for all $d>0$ and $\ell>0$

$$
\int Q_{u u^{\prime}} \mathrm{d} u^{\prime} \leq\left(1+\pi^{-2}\right) \frac{-\Delta_{u}^{\mathcal{N}}}{-\Delta_{u}^{\mathcal{N}}+(d \ell)^{-2}}
$$

Proof. It is enough to consider $\ell=1$. Let $-\Delta_{u u^{\prime}}^{\mathcal{N}}$ denote the Neumann Laplacian in the box $B\left(u, u^{\prime}\right)$. Observe that since $B\left(u, u^{\prime}\right) \subseteq \widetilde{B}\left(u^{\prime}\right)$, the Neumann Laplacian $-\Delta_{u u^{\prime}}^{\mathcal{N}}$ has a gap of at least $\pi^{2} d^{-2}$, i.e., $-\Delta_{u u^{\prime}}^{\mathcal{N}} \geq \pi^{2} d^{-2} Q_{u u^{\prime}}$. Thus

$$
Q_{u u^{\prime}} \leq\left(1+\pi^{-2}\right) \frac{-\Delta_{u u^{\prime}}^{\mathcal{N}}}{-\Delta_{u u^{\prime}}^{\mathcal{N}}+d^{-2}}
$$

The same argument that led to 44 gives

$$
\int \frac{-\Delta_{u u^{\prime}}^{\mathcal{N}}}{-\Delta_{u u^{\prime}}^{\mathcal{N}}+d^{-2}} \mathrm{~d} u^{\prime} \leq \frac{-\Delta_{u}^{\mathcal{N}}}{-\Delta_{u}^{\mathcal{N}}+d^{-2}},
$$

which concludes the proof of the lemma.

This lemma shows that the first term in 41, after localization, leads to a gap in the small boxes. The full kinetic energy localization is given in the next lemma.

LEMMA 3.9 (Small-box kinetic energy localization). Let $\widehat{\mathcal{T}}_{u}$ be the replacement for the 
kinetic energy given in (41) in terms of the parameters $s, d, \varepsilon_{T}$ and the constant $b$. Let

$$
\mathcal{T}_{u u^{\prime}}=\varepsilon_{T}\left(1+\pi^{-2}\right)^{-1}(d \ell)^{-2} Q_{u u^{\prime}}+Q_{u u^{\prime}} \chi_{u} \widetilde{\chi}_{u^{\prime}}\left[\sqrt{-\Delta}-(d s \ell)^{-1}\right]_{+}^{2} \widetilde{\chi}_{u^{\prime}} \chi_{u} Q_{u u^{\prime}}
$$

Our assertion is that if $\left(s^{-2}+d^{-3}\right)(d s)^{-2} s^{M} \leq \delta$, then, for all $0<\varepsilon_{T}<1$,

$$
\widehat{\mathcal{T}}_{u}-\frac{b}{2} \ell^{-2} Q_{u} \geq \int \mathcal{T}_{u u^{\prime}} \mathrm{d} u^{\prime}
$$

The first term in (48) yields a gap above zero of size $\varepsilon_{T}\left(1+\pi^{-2}\right)^{-1}(d \ell)^{-2}$ in the spectrum of $\mathcal{T}_{u u^{\prime}}$, which we will refer to as the Neumann gap.

Proof. We again take $\ell=1$. The integral over the first term in $\mathcal{T}_{u u^{\prime}}$ is bounded above by the first term in $\widehat{\mathcal{T}}_{u}$ by Lemma 3.8. We concentrate on the second term in $\mathcal{T}_{u u^{\prime}}$. By a unitary transformation $(x \mapsto x / d$ and $p \mapsto p d)$ of the result in Lemma 3.5 we obtain

$$
\begin{aligned}
& \int \widetilde{Q}_{u^{\prime}} \widetilde{\chi}_{u^{\prime}}\left[\sqrt{-\Delta}-(d s)^{-1}\right]_{+}^{2} \widetilde{\chi}_{u^{\prime}} \widetilde{Q}_{u^{\prime}} \mathrm{d} u^{\prime} \leq d^{-2} F_{s}(d \sqrt{-\Delta}) \\
& \leq\left(1-\varepsilon_{T}\right)\left[\sqrt{-\Delta}-\frac{1}{2} s^{-1}\right]_{+}^{2}+\varepsilon_{T}\left[\sqrt{-\Delta}-\frac{1}{2}(d s)^{-1}\right]_{+}^{2}+C(d s)^{-2} s^{M-2},
\end{aligned}
$$

where the function $F_{s}$ is given in $(33)$ and we used that $\left[\sqrt{-\Delta}-\frac{1}{2}(d s)^{-1}\right]_{+}^{2} \leq\left[\sqrt{-\Delta}-\frac{1}{2} s^{-1}\right]_{+}^{2}$. Thus the proof would be complete if the operator appearing as the integrand in the second term in $\mathcal{T}_{u u^{\prime}}$ would have been, instead,

$$
Q_{u} \chi_{u} \widetilde{Q}_{u^{\prime}} \widetilde{\chi}_{u^{\prime}}\left[\sqrt{-\Delta}-(d s)^{-1}\right]_{+}^{2} \widetilde{\chi}_{u^{\prime}} \widetilde{Q}_{u^{\prime}} \chi_{u} Q_{u}
$$

We will estimate the difference between these operators, which is

$$
\begin{aligned}
D= & Q_{u} \chi_{u} \widetilde{Q}_{u^{\prime}} \widetilde{\chi}_{u^{\prime}}\left[\sqrt{-\Delta}-(d s)^{-1}\right]_{+}^{2} \widetilde{\chi}_{u^{\prime}} \widetilde{Q}_{u^{\prime}} \chi_{u} Q_{u} \\
& -Q_{u u^{\prime}} \chi_{u} \widetilde{\chi}_{u^{\prime}}\left[\sqrt{-\Delta}-(d s)^{-1}\right]_{+}^{2} \widetilde{\chi}_{u^{\prime}} \chi_{u} Q_{u u^{\prime}} \\
= & Q_{u}\left(\chi_{u} \widetilde{Q}_{u^{\prime}}-Q_{u u^{\prime}} \chi_{u}\right) \widetilde{\chi}_{u^{\prime}}\left[\sqrt{-\Delta}-(d s)^{-1}\right]_{+}^{2} \widetilde{\chi}_{u^{\prime}} \widetilde{Q}_{u^{\prime}} \chi_{u} Q_{u} \\
& +Q_{u u^{\prime}} \chi_{u} \widetilde{\chi}_{u^{\prime}}\left[\sqrt{-\Delta}-(d s)^{-1}\right]_{+}^{2} \widetilde{\chi}_{u^{\prime}}\left(\widetilde{Q}_{u^{\prime}} \chi_{u}-\chi_{u} Q_{u u^{\prime}}\right) Q_{u}
\end{aligned}
$$

where we have used that $Q_{u} Q_{u u^{\prime}}=Q_{u u^{\prime}}$. We observe that (using Dirac notation)

$$
\begin{aligned}
\left(\chi_{u} \widetilde{Q}_{u^{\prime}}-Q_{u u^{\prime}} \chi_{u}\right) \widetilde{\chi}_{u^{\prime}}= & \chi_{u} \widetilde{\theta}_{u^{\prime}} \widetilde{\chi}_{u^{\prime}}-d^{-3} \chi_{u}\left|\widetilde{\theta}_{u^{\prime}}\right\rangle\left\langle\widetilde{\theta}_{u^{\prime}}\right| \widetilde{\chi}_{u^{\prime}}-\theta_{u} \widetilde{\theta}_{u^{\prime}} \chi_{u} \widetilde{\chi}_{u^{\prime}} \\
& +\left|B\left(u, u^{\prime}\right)\right|^{-1}\left|\theta_{u} \widetilde{\theta}_{u^{\prime}}\right\rangle\left\langle\theta_{u} \widetilde{\theta}_{u^{\prime}}\right| \chi_{u} \widetilde{\chi}_{u^{\prime}}
\end{aligned}
$$




$$
=\left|B\left(u, u^{\prime}\right)\right|^{-1}\left|\theta_{u} \widetilde{\theta}_{u^{\prime}}\right\rangle\left\langle\chi_{u} \widetilde{\chi}_{u^{\prime}}\left|-d^{-3}\right| \chi_{u} \widetilde{\theta}_{u^{\prime}}\right\rangle\left\langle\widetilde{\chi}_{u^{\prime}}\right|
$$

exploiting the facts that $\widetilde{\theta}_{u^{\prime}} \widetilde{\chi}_{u^{\prime}}=\widetilde{\chi}_{u^{\prime}}$ and $\theta_{u} \chi_{u}=\chi_{u}$. It is now simple to estimate the operator norm of $D$

$$
\begin{aligned}
\|D\| \leq & C\left\|\left(\chi_{u} \widetilde{Q}_{u^{\prime}}-Q_{u u^{\prime}} \chi_{u}\right) \widetilde{\chi}_{u^{\prime}}\left[\sqrt{-\Delta}-(d s)^{-1}\right]_{+}^{2}\right\| \\
\leq & C\left|B\left(u, u^{\prime}\right)\right|^{-1 / 2}\left(\int_{|p|>(d s)^{-1}}|p|^{4}\left|\widehat{\chi_{u} \widetilde{\chi}_{u^{\prime}}}(p)\right|^{2} \mathrm{~d} p\right)^{1 / 2} \\
& +C d^{-3 / 2}\left(\int_{|p|>(d s)^{-1}}|p|^{4}\left|\widehat{\widetilde{\chi}_{u^{\prime}}}(p)\right|^{2} \mathrm{~d} p\right)^{1 / 2} \\
\leq & C(d s)^{M-2}\left|B\left(u, u^{\prime}\right)\right|^{-1 / 2}\left\langle\chi_{u} \widetilde{\chi}_{u^{\prime}} \mid(-\Delta)^{M} \chi_{u} \widetilde{\chi}_{u^{\prime}}\right\rangle^{1 / 2}+C(d s)^{M-2} d^{-3 / 2}\left\langle\widetilde{\chi}_{u^{\prime}} \mid(-\Delta)^{M} \widetilde{\chi}_{u^{\prime}}\right\rangle^{1 / 2} \\
\leq & C(d s)^{M-2} d^{-M} \leq C(d s)^{-2} s^{M} .
\end{aligned}
$$

Hence $D=Q_{u} D Q_{u} \geq-C(d s)^{-2} s^{M} Q_{u}$ and

$$
\begin{aligned}
\int & Q_{u u^{\prime}} \chi_{u} \widetilde{\chi}_{u^{\prime}}\left[\sqrt{-\Delta}-(d s)^{-1}\right]_{+}^{2} \widetilde{\chi}_{u^{\prime}} \chi_{u} Q_{u u^{\prime}} \mathrm{d} u^{\prime} \\
= & \int Q_{u} \chi_{u} \widetilde{Q}_{u^{\prime}} \widetilde{\chi}_{u^{\prime}}\left[\sqrt{-\Delta}-(d s)^{-1}\right]_{+}^{2} \widetilde{\chi}_{u^{\prime}} \widetilde{Q}_{u^{\prime}} \chi_{u} Q_{u} \mathrm{~d} u^{\prime}-\int_{\left\{u^{\prime} \mid B(u) \cap \widetilde{B}\left(u^{\prime}\right) \neq \emptyset\right\}} D \mathrm{~d} u^{\prime} \\
\leq & Q_{u} \chi_{u}\left(\left(1-\varepsilon_{T}\right)\left[\sqrt{-\Delta}-\frac{1}{2} s^{-1}\right]_{+}^{2}+\varepsilon_{T}\left[\sqrt{-\Delta}-\frac{1}{2}(d s)^{-1}\right]_{+}^{2}\right) \chi_{u} Q_{u} \\
& +C\left(s^{-2}+d^{-3}\right)(d s)^{-2} s^{M} Q_{u} .
\end{aligned}
$$

We have here used the fact that the volume of $\left\{u^{\prime} \mid B(u) \cap \widetilde{B}\left(u^{\prime}\right) \neq \emptyset\right\}$ is bounded by $C d^{-3}$.

We shall throughout the rest of the paper assume that the conditions in Lemmas 3.7 and 3.9 are satisfied.

CONDITION 2: In terms of the integer $M$ appearing in the definition (13) of $\chi$ we have

$$
0<\varepsilon_{T}<\delta, \quad \text { and } \quad\left(s^{-2}+d^{-3}\right)(d s)^{-2} s^{M} \leq \delta
$$

where $\delta$ was introduced in Condition 1 to ensure adequate smallness of relevant parameters. This shows that we need $M \geq 8$.

A stronger upper bound on $\varepsilon_{T}$ will be required in Condition 3 . 


\subsection{Localization of the Total Energy}

We define the localized Hamiltonian, $H_{u}$, in a "large" box $B(u)$ to be

$$
H_{u}=\sum_{i=1}^{N}\left(\left(1-\varepsilon_{0}\right) \widehat{\mathcal{T}}_{u, i}-\rho \int w_{B(u)}\left(x_{i}, y\right) \mathrm{d} y\right)+\sum_{1 \leq i<j \leq N} w_{B(u)}\left(x_{i}, x_{j}\right)+\frac{1}{2} \rho^{2} \iint w_{B(u)}(x, y) \mathrm{d} x \mathrm{~d} y,
$$

where $0 \leq \varepsilon_{0} \leq 1 / 2$ is a parameter to be determined later. The subscript $i$, as usual, refers to the $i^{\text {th }}$ particle. Recall that $\widehat{\mathcal{T}}_{u}$ was defined in (41) and $w_{B(u)}$ was defined in (21). The Hamiltonian $H_{u}$ is defined as a quadratic form on the Bosonic Fock space $\mathcal{F}_{B}\left(H_{0}^{1}(\Lambda)\right.$ ).

The localized Hamiltonian in a "small" box $B\left(u, u^{\prime}\right)$ is

$$
\begin{aligned}
H_{u u^{\prime}}= & \sum_{i=1}^{N}\left(\left(1-\varepsilon_{0}\right) \mathcal{T}_{u u^{\prime}, i}-\rho \int w_{B\left(u, u^{\prime}\right)}\left(x_{i}, y\right) \mathrm{d} y\right)+\sum_{1 \leq i<j \leq N} w_{B\left(u, u^{\prime}\right)}\left(x_{i}, x_{j}\right) \\
& +\frac{1}{2} \rho^{2} \iint w_{B\left(u, u^{\prime}\right)}(x, y) \mathrm{d} x \mathrm{~d} y,
\end{aligned}
$$

where $w_{B\left(u, u^{\prime}\right)}$ was defined in (21) and $\mathcal{T}_{u u^{\prime}}$ was defined in (48). The results of Proposition 3.1 , (42) and Lemmas 3.7 and 3.9 can be combined to give our final localization estimate.

THEOREM 3.10 (Main localization inequalities).

If $M \geq 8$, Condition 1 and Condition 2 are satisfied, then we have for all $0 \leq \varepsilon_{0} \leq 1 / 2$

$$
H_{\rho} \geq \int_{\ell^{-1} \Lambda^{\prime}}\left(-\varepsilon_{0} \Delta_{u}^{\mathcal{N}}+H_{u}\right) \mathrm{d} u, \quad \text { and, for all } u \in \mathbb{R}^{3}, \quad H_{u}-\frac{b}{2} \ell^{-2} Q_{u} \geq \int_{\mathbb{R}^{3}} H_{u u^{\prime}} \mathrm{d} u^{\prime},
$$

where $\Lambda^{\prime}=\Lambda+\left[-\frac{\ell}{2}, \frac{\ell}{2}\right]^{3}$ and $H_{\rho}$ was introduced in (9).

We introduce the notation

$$
H_{B}=\left\{\begin{array}{ll}
H_{u}, & \text { if } B=B(u) \\
H_{u u^{\prime}}, & \text { if } B=B\left(u, u^{\prime}\right)
\end{array} \quad \text { and } \quad \mathcal{T}_{B}= \begin{cases}\left(1-\varepsilon_{0}\right) \widehat{\mathcal{T}}_{u}, & \text { if } B=B(u) \\
\left(1-\varepsilon_{0}\right) \mathcal{T}_{u u^{\prime}}, & \text { if } B=B\left(u, u^{\prime}\right) .\end{cases}\right.
$$

The reason for the above $\left(1-\varepsilon_{0}\right)$-term is that we still need some kinetic energy in the end of this paper when we want to apply Lemma 6.8. With the corresponding notations for $w_{B}$ in (21) the box Hamiltonian can be written

$$
H_{B}=\sum_{i=1}^{N}\left(\mathcal{T}_{B, i}-\rho \int w_{B}\left(x_{i}, y\right) \mathrm{d} y\right)+\sum_{1 \leq i<j \leq N} w_{B}\left(x_{i}, x_{j}\right)+\frac{1}{2} \rho^{2} \iint w_{B}(x, y) \mathrm{d} x \mathrm{~d} y .
$$




\section{Energy in a Single Box}

In this section we will study the energy in a single box $B$, i.e., the ground state energy of the Hamiltonian $H_{B}$. We denote by $P_{B}$ the orthogonal projection onto the characteristic function of $B$ and by $Q_{B}$ the projection orthogonal to constant functions in $B$. In particular, $P_{B}+Q_{B}=\mathbb{1}_{B}$ is the projection onto the subspace of functions supported on $B$. We define the operators

$$
n=\sum_{i=1}^{N} \mathbb{1}_{B, i}, \quad n_{0}=\sum_{i=1}^{N} P_{B, i}, \quad n_{+}=\sum_{i=1}^{N} Q_{B, i} .
$$

Here $n$ represents the number of particles in the box $B, n_{0}$ the number of particles in the constant function, which we will refer to as the condensate particles, and $n_{+}$the number of particles not in the condensate, which we will refer to as the excited particles. We have $n=n_{+}+n_{0}$.

The particle number operator $n$ commutes with the box operator $H_{B}$, but $n_{+}$and $n_{0}$ do not commute with $H_{B}$. In our discussion below we may assume that $n$ is a parameter, i.e., we restrict to eigenspaces for the operator $n$. We shall not distinguish between the operator $n$ and its eigenvalues.

We give a simple a priori bound on $n_{+}$, which will be improved later.

LEMMA 4.1 (Simple bound on the ground state energy of $H_{B}$ and on $n_{+}$).

The ground state energy $E_{B}$ of $H_{B}$ satisfies

$$
0>E_{B} \geq-C n \rho|B| \mathcal{U}_{B}
$$

with $\mathcal{U}_{B}$ given in (22). Moreover, if $B=B\left(u, u^{\prime}\right)$, for any $n$-particle state with expectation value $\left\langle H_{B}\right\rangle \leq \frac{1}{2} \rho^{2} \iint w_{B}(x, y) \mathrm{d} x \mathrm{~d} y=\rho^{2}|B|^{2} \mathcal{U}_{B}$ we have

$$
\left\langle n_{+}\right\rangle \leq C\left(1-\varepsilon_{0}\right)^{-1} \varepsilon_{T}^{-1} \rho a(d \ell)^{2} n \max \chi_{B}^{2}
$$

Proof. The upper bound on $E_{B}$ follows by using a trial state in which all particles are in the condensate, i.e., an eigenstate of $n_{+}$with eigenvalue 0 . If there are $n$ particles, we find that the expectation value in this state is

$$
\left\langle H_{B}\right\rangle= \begin{cases}{\left[-|B|^{-1} \rho+\frac{1}{2} \rho^{2}\right] \iint w_{B}(x, y) \mathrm{d} x \mathrm{~d} y} & \text { for } n=1 \\ \frac{1}{2}\left[(n-\rho|B|)^{2}-n\right]|B|^{-2} \iint w_{B}(x, y) \mathrm{d} x \mathrm{~d} y & \text { for } n \geq 2\end{cases}
$$

We can choose $n=1$ if $\rho|B|<2$ and otherwise choose $n \geq 2$ such that $|n-\rho| B||<1$. 
Hence $\left\langle H_{B}\right\rangle<0$ and thus the ground state energy of $H_{B}$ is negative. The lower bound on $E_{B}$ follows immediately from (24) since $\mathcal{T}_{B}$ and $w_{B}$ are non-negative.

To obtain (56): If an $n$-particle state satisfies $\left\langle H_{B}\right\rangle \leq \frac{1}{2} \rho^{2} \iint w_{B}(x, y) \mathrm{d} x \mathrm{~d} y$, we have

$$
0 \geq \sum_{i=1}^{N}\left\langle\mathcal{T}_{B, i}-\rho \int w_{B}\left(x_{i}, y\right) \mathrm{d} y\right\rangle \geq\left(1-\varepsilon_{0}\right) \varepsilon_{T}\left(1+\pi^{-2}\right)^{-1}(d \ell)^{-2}\left\langle n_{+}\right\rangle-C \rho a n \max \chi_{B}^{2}
$$

where we have used 48 and that $\max _{x} \int \omega_{B}(x, y) \mathrm{d} y \leq C a \max \chi_{B}^{2}$ by Lemma 3.2 .

\subsection{The Negligible (Non-Quadratic) Parts of the Potential}

We treat the potential energy terms in $H_{B}$ according to how many excited particles they involve. We write $\mathbb{1}_{B}=P_{B}+Q_{B}$ and we expand and classify the terms according to the number of $Q$-factors, no- $Q, 1-Q, \ldots, 4$ - $Q$. In the following we will simply write $P_{B}=P$ and $Q_{B}=Q$.

no- $\mathcal{Q}$ terms:

$$
\begin{aligned}
\mathcal{Q}_{0} & :=-\sum_{i} \rho P_{i} \int w_{B}\left(x_{i}, y\right) \mathrm{d} y P_{i}+\sum_{i<j} P_{i} P_{j} w_{B}\left(x_{i}, x_{j}\right) P_{i} P_{j}+\frac{1}{2} \rho^{2} \iint w_{B}(x, y) \mathrm{d} x \mathrm{~d} y \\
& =\left[\left(n_{0}-\rho|B|\right)^{2}-n_{0}\right] \mathcal{U}_{B}=\left[(n-\rho|B|)^{2}-2(n-\rho|B|) n_{+}+n_{+}^{2}-n_{0}\right] \mathcal{U}_{B},
\end{aligned}
$$

where we have used the notation 22 .

\section{1-Q $\mathcal{Q}$ terms:}

$$
\begin{aligned}
\mathcal{Q}_{1} & :=\sum_{i, j} P_{i} P_{j} w_{B}\left(x_{i}, x_{j}\right) Q_{i} P_{j}-\sum_{i} \rho P_{i} \int w_{B}\left(x_{i}, y\right) \mathrm{d} y Q_{i}+\text { h.c. } \\
& =\sum_{i} P_{i} \int w_{B}\left(x_{i}, y\right) \mathrm{d} y Q_{i}\left(n_{0}|B|^{-1}-\rho\right)+\text { h.c. } \\
& =\mathcal{Q}_{1}^{\prime}+\mathcal{Q}_{1}^{\prime \prime}
\end{aligned}
$$

where

$$
\mathcal{Q}_{1}^{\prime}:=(n-\rho|B|)|B|^{-1}\left(\sum_{i} P_{i} \int w_{B}\left(x_{i}, y\right) \mathrm{d} y Q_{i}+\sum_{i} Q_{i} \int w_{B}\left(x_{i}, y\right) \mathrm{d} y P_{i}\right)
$$


and

$$
\mathcal{Q}_{1}^{\prime \prime}:=-|B|^{-1} \sum_{i} P_{i} \int w_{B}\left(x_{i}, y\right) \mathrm{d} y Q_{i} n_{+}-|B|^{-1} n_{+} \sum_{i} Q_{i} \int w_{B}\left(x_{i}, y\right) \mathrm{d} y P_{i}
$$

LEMMA 4.2 (Estimates on $\mathcal{Q}_{1}$ ). For all $\varepsilon_{1}^{\prime}, \varepsilon_{1}^{\prime \prime}>0$

$$
\mathcal{Q}_{1}^{\prime} \geq-|n-\rho| B||\left(\varepsilon_{1}^{\prime} n_{0}+\varepsilon_{1}^{\prime-1} C n_{+}\right) \mathcal{U}_{B} \quad \text { and } \quad \mathcal{Q}_{1}^{\prime \prime} \geq-\left(\varepsilon_{1}^{\prime \prime}\left(n_{+}+1\right) n_{0}+C \varepsilon_{1}^{\prime \prime-1} n_{+}^{2}\right) \mathcal{U}_{B}
$$

Proof. We prove first the bound on $\mathcal{Q}_{1}^{\prime \prime}$. We have

$$
\mathcal{Q}_{1}^{\prime \prime}=-\sqrt{n_{+}+1} \sum_{i} P_{i} \int w_{B}\left(x_{i}, y\right) \mathrm{d} y Q_{i} \sqrt{n_{+}}|B|^{-1}+\text { h.c. }
$$

since for any self-adjoint operator $A$ we get $\sum_{i} P_{i} A_{i} Q_{i} n_{+}=\left(n_{+}+1\right) \sum_{i} P_{i} A_{i} Q_{i}$ and hence $\sum_{i} P_{i} A_{i} Q_{i} \sqrt{n_{+}}=\sqrt{n_{+}+1} \sum_{i} P_{i} A_{i} Q_{i}$.

Since $w_{B} \geq 0$, we obtain from a Schwarz inequality and Lemma 3.2 that

$$
\mathcal{Q}_{1}^{\prime \prime} \geq-\left(\varepsilon_{1}^{\prime \prime}\left(n_{+}+1\right) \sum_{i} P_{i}+C \varepsilon_{1}^{\prime \prime-1} n_{+} \sum_{i} Q_{i}\right) \mathcal{U}_{B} .
$$

The estimate on $\mathcal{Q}_{1}^{\prime}$ follows by applying a similar Schwarz inequality.

2- $\mathcal{Q}$ terms: There are two kinds of $2-\mathcal{Q}$ terms. There are terms which contribute to the energy to the order of interest. They will primarily be treated together with the quadratic Hamiltonian later on. The remaining $2-\mathcal{Q}$ terms are negligible error terms that we will estimate here.

The 2 - $\mathcal{Q}$ terms that will later appear in the quadratic Hamiltonian are

$$
\mathcal{Q}_{2}^{\prime}:=\sum_{i, j} P_{i} Q_{j} w_{B}\left(x_{i}, x_{j}\right) P_{j} Q_{i}+\sum_{i<j}\left(Q_{i} Q_{j} w_{B}\left(x_{i}, x_{j}\right) P_{j} P_{i}+P_{j} P_{i} w_{B}\left(x_{i}, x_{j}\right) Q_{i} Q_{j}\right) .
$$

We shall however give an a priori estimate on these terms already now. This estimate will be used in Case II in the proof of Lemma 5.2. Using the estimate $0 \leq w_{B} \leq C a R^{-3} \max \chi_{B}^{2}$ and the Schwarz inequality twice gives that

$$
\begin{aligned}
\mathcal{Q}_{2}^{\prime} & \geq-\sum_{i, j} P_{i} Q_{j} w_{B}\left(x_{i}, x_{j}\right) P_{i} Q_{j}-\sum_{i<j}\left(2 Q_{i} Q_{j} w_{B}\left(x_{i}, x_{j}\right) Q_{j} Q_{i}+\frac{1}{2} P_{j} P_{i} w_{B}\left(x_{i}, x_{j}\right) P_{i} P_{j}\right) \\
& \geq-n_{0}|B|^{-1} \sum_{i} Q_{i} \int w_{B}\left(x_{i}, y\right) \mathrm{d} y Q_{i}-C n_{+}^{2} a R^{-3} \max \chi_{B}^{2}-\frac{1}{2} \sum_{i<j} P_{j} P_{i} w_{B}\left(x_{i}, x_{j}\right) P_{i} P_{j}
\end{aligned}
$$




$$
\geq-C n n_{+} \mathcal{U}_{B}-C n_{+}^{2} a R^{-3} \max \chi_{B}^{2}-\frac{1}{2} n^{2} \mathcal{U}_{B}
$$

In the last inequality we have used that $\sum_{i} Q_{i} \int \omega_{B}\left(x_{i}, y\right) \mathrm{d} y Q_{i} \leq C|B| \mathcal{U}_{B} n_{+}$by 24 since $\sum_{i} Q_{i} \int \omega_{B}\left(x_{i}, y\right) \mathrm{d} y Q_{i}$ commutes with $n_{+}$. The negligible $2-\mathcal{Q}$ terms are estimated in the same way

$$
\begin{aligned}
\mathcal{Q}_{2}^{\prime \prime} & :=-\sum_{i} \rho Q_{i} \int w_{B}\left(x_{i}, y\right) \mathrm{d} y Q_{i}+\sum_{i, j} Q_{i} P_{j} w_{B}\left(x_{i}, x_{j}\right) P_{j} Q_{i} \\
& =\left(n_{0}-\rho|B|\right)|B|^{-1} \sum_{i} Q_{i} \int w_{B}\left(x_{i}, y\right) \mathrm{d} y Q_{i} \\
& =\left(n-\rho|B|-n_{+}\right)|B|^{-1} \sum_{i} Q_{i} \int w_{B}\left(x_{i}, y\right) \mathrm{d} y Q_{i} \geq-C\left([\rho|B|-n]_{+} n_{+}+n_{+}^{2}\right) \mathcal{U}_{B} .
\end{aligned}
$$

3-Q $\mathcal{Q}$ terms: For all $\varepsilon_{3}>0$

$$
\begin{aligned}
\mathcal{Q}_{3} & :=\sum_{i, j} P_{j} Q_{i} w_{B}\left(x_{i}, x_{j}\right) Q_{i} Q_{j}+\text { h.c. } \\
& \geq-\sum_{i \neq j}\left(2 \varepsilon_{3}^{-1} P_{j} Q_{i} w_{B}\left(x_{i}, x_{j}\right) Q_{i} P_{j}+\frac{\varepsilon_{3}}{2} Q_{j} Q_{i} w_{B}\left(x_{i}, x_{j}\right) Q_{i} Q_{j}\right) \\
& \geq-C \varepsilon_{3}^{-1} n n_{+} \mathcal{U}_{B}-\varepsilon_{3} \sum_{i<j} Q_{j} Q_{i} w_{B}\left(x_{i}, x_{j}\right) Q_{i} Q_{j} .
\end{aligned}
$$

The first inequality uses a Schwarz inequality, while the second inequality uses Lemma 3.2 and the fact that $n_{0} \leq n$. Note that the above estimates are given as lower bounds, but that we of course also could have stated them as two-sided bounds. The last term above can be absorbed into the positive 4 - $\mathcal{Q}$ term if $\varepsilon_{3} \leq 1$. We will do this initially, but at a later stage in the proof we have to control the first term in 64 by choosing $\varepsilon_{3} \gg 1$. At that time we will have good control over the number of excited particles, $n_{+}$, in the large box. The second term in (64) will then be controlled by applying Lemma 6.8, where we apply the kinetic energy term $-\varepsilon_{0} \Delta_{u}^{\mathcal{N}}$ that we have saved in Theorem 3.10 for exactly this purpose.

4- $\mathcal{Q}$ term: This is the positive term

$$
\mathcal{Q}_{4}:=\sum_{i<j} Q_{j} Q_{i} w_{B}\left(x_{i}, x_{j}\right) Q_{i} Q_{j}
$$

and for a lower bound it can be ignored or used to control other errors. We will only need an estimate on the $4-\mathcal{Q}$ term in a large box $B=B(u)$ as explained above. 


\subsection{The Quadratic Hamiltonian}

We can write the box Hamiltonian as

$$
H_{B}=\sum_{i=1}^{N} \mathcal{T}_{B, i}+\mathcal{Q}_{0}+\mathcal{Q}_{1}^{\prime}+\mathcal{Q}_{1}^{\prime \prime}+\mathcal{Q}_{2}^{\prime}+\mathcal{Q}_{2}^{\prime \prime}+\mathcal{Q}_{3}+\mathcal{Q}_{4}
$$

We have estimated all terms except the quadratic part $\sum_{i=1}^{N} \mathcal{T}_{B, i}+\mathcal{Q}_{2}^{\prime}$.

We first consider the localized kinetic energy.

For $B=B(u)$, i.e., a large box we have from (41) and (53) that

$$
\mathcal{T}_{B}=\left(1-\varepsilon_{0}\right) \varepsilon_{T}(d \ell)^{-2} \frac{-\Delta_{u}^{\mathcal{N}}}{-\Delta_{u}^{\mathcal{N}}+(d \ell)^{-2}}+\left(1-\varepsilon_{0}\right) b \ell^{-2} Q+Q \chi_{B} \tau_{B}(-\Delta) \chi_{B} Q
$$

with

$$
\tau_{B}\left(k^{2}\right)=\left(1-\varepsilon_{0}\right)\left(1-\varepsilon_{T}\right)\left[|k|-\frac{1}{2}(s \ell)^{-1}\right]_{+}^{2}+\left(1-\varepsilon_{0}\right) \varepsilon_{T}\left[|k|-\frac{1}{2}(d s \ell)^{-1}\right]_{+}^{2} .
$$

For $B=B\left(u, u^{\prime}\right)$, i.e., a small box we have from 48 and 53 that

$$
\mathcal{T}_{B}=\left(1-\varepsilon_{0}\right) \varepsilon_{T}\left(1+\pi^{-2}\right)^{-1}(d \ell)^{-2} Q+Q \chi_{B} \tau_{B}(-\Delta) \chi_{B} Q
$$

with

$$
\tau_{B}\left(k^{2}\right)=\left(1-\varepsilon_{0}\right)\left[|k|-(d s \ell)^{-1}\right]_{+}^{2} .
$$

The interesting part of $\mathcal{T}_{B}$ is the term of form $Q \chi_{B} \tau_{B}(-\Delta) \chi_{B} Q$. The other terms, which are positive, are only used to control errors. We put them aside for the moment and define the quadratic Hamiltonian

$$
H_{\mathrm{Quad}}=\sum_{i=1}^{N}\left(Q \chi_{B} \tau_{B}(-\Delta) \chi_{B} Q\right)_{i}+\mathcal{Q}_{2}^{\prime}
$$

At the end of this paper it will be useful to treat the term $\mathcal{Q}_{1}^{\prime}$ together with $H_{\text {Quad }}$.

To handle $H_{\text {Quad }}$, we use the formalism of second quantization. For all $k \in \mathbb{R}^{3}$ we define the operator

$$
b_{k}=a_{0}^{*} a\left(Q\left(e^{i k x} \chi_{B}\right)\right)
$$

where $a\left(Q\left(e^{i k x} \chi_{B}\right)\right)$ is the operator that annihilates an exited particle in the state given by the function $Q\left(e^{i k x} \chi_{B}\right) \in L^{2}(B)$, and $a_{0}$ is the operator that creates a particle in the condensate. These two operators commute. Note that $b_{k}$ is a bounded operator when restricted to a 
subspace of finite $n$. Its adjoint is

$$
b_{k}^{*}=a\left(Q\left(e^{i k x} \chi_{B}\right)\right)^{*} a_{0} .
$$

Since $a_{0}^{*}$ commutes with $a\left(Q\left(e^{i k x} \chi_{B}\right)\right)$, we have the commutation relations

$$
\left[b_{k}, b_{k^{\prime}}\right]=0, \quad\left[b_{k}, b_{k^{\prime}}^{*}\right]=a_{0}^{*} a_{0}\left\langle Q\left(e^{i k x} \chi_{B}\right) \mid Q\left(e^{i k^{\prime} x} \chi_{B}\right)\right\rangle-a\left(Q\left(e^{i k^{\prime} x} \chi_{B}\right)\right)^{*} a\left(Q\left(e^{i k x} \chi_{B}\right)\right)
$$

for all $k, k^{\prime} \in \mathbb{R}^{3}$. In particular,

$$
\left[b_{k}, b_{k}^{*}\right] \leq a_{0}^{*} a_{0} \int \chi_{B}^{2}=n_{0} \int \chi_{B}^{2}
$$

The term $Q \chi_{B} \tau_{B}(-\Delta) \chi_{B} Q$ and its second quantization can be written

$$
\begin{aligned}
Q \chi_{B} \tau_{B}(-\Delta) \chi_{B} Q & =(2 \pi)^{-3} \int_{\mathbb{R}^{3}} \tau_{B}\left(k^{2}\right)\left|Q\left(\chi_{B} e^{i k x}\right)\right\rangle\left\langle Q\left(\chi_{B} e^{i k x}\right)\right| \mathrm{d} k \\
\stackrel{2^{\text {nd }} \longrightarrow \text { quant }}{\longrightarrow} & (2 \pi)^{-3} \int_{\mathbb{R}^{3}} \tau_{B}\left(k^{2}\right) a\left(Q\left(\chi_{B} e^{i k x}\right)\right)^{*} a\left(Q\left(\chi_{B} e^{i k x}\right)\right) \mathrm{d} k \\
& \geq(2 \pi)^{-3} \int_{\mathbb{R}^{3}} \tau_{B}\left(k^{2}\right) a\left(Q\left(\chi_{B} e^{i k x}\right)\right)^{*} \frac{a_{0} a_{0}^{*}}{n} a\left(Q\left(\chi_{B} e^{i k x}\right)\right) \mathrm{d} k \\
& =(2 \pi)^{-3} n^{-1} \int_{\mathbb{R}^{3}} \tau_{B}\left(k^{2}\right) b_{k}^{*} b_{k} \mathrm{~d} k .
\end{aligned}
$$

Here we used that $b_{k} \psi=0$ if $\psi$ is in the condensate allowing us to assume that $n_{+} \geq 1$ such that in fact $a_{0} a_{0}^{*} \leq n$. Likewise we may write

$$
\begin{aligned}
\mathcal{Q}_{2}^{\prime}= & \frac{1}{2}(2 \pi)^{-3}|B|^{-1} \int \widehat{W}(k)\left(b_{k}^{*} b_{k}+b_{-k}^{*} b_{-k}+b_{k}^{*} b_{-k}^{*}+b_{k} b_{-k}\right) \mathrm{d} k \\
& -(2 \pi)^{-3}|B|^{-1} \int \widehat{W}(k) a\left(Q\left(\chi_{B} e^{i k x}\right)\right)^{*} a\left(Q\left(\chi_{B} e^{i k x}\right)\right) \mathrm{d} k
\end{aligned}
$$

and

$$
\mathcal{Q}_{1}^{\prime}=(n-\rho|B|)(2 \pi)^{-3}|B|^{-3 / 2} \int \widehat{W}(k)\left(\overline{\widehat{\chi}_{B}(k)} b_{k}+\widehat{\chi}_{B}(k) b_{k}^{*}\right) \mathrm{d} k
$$

The last term in $\mathcal{Q}_{2}^{\prime}$ may be written

$$
(2 \pi)^{-3}|B|^{-1} \int \widehat{W}(k) a\left(Q\left(\chi_{B} e^{i k x}\right)\right)^{*} a\left(Q\left(\chi_{B} e^{i k x}\right)\right) \mathrm{d} k=\sum_{i=1}^{N} Q_{i} Z_{i} Q_{i}
$$


where $Z$ is the operator with integral kernel

$$
k_{Z}(x, y)=|B|^{-1} \chi_{B}(x) W(x-y) \chi_{B}(y) .
$$

LEMMA 4.3. The operator $Z$ on $L^{2}\left(\mathbb{R}^{3}\right)$ with integral kernel 80 satisfies the bound

$$
\|Z\| \leq C a \min \left\{R^{-3},|B|^{-1}\right\} \max \chi_{B}^{2}
$$

In particular, if $B=B(u)$, we have

$$
\|Z\| \leq C a|B|^{-1}
$$

Proof. It is clear that

$$
\|Z\| \leq|B|^{-1} \max \chi_{B}^{2} \int W \leq C|B|^{-1} \max \chi_{B}^{2} \int v_{1} \leq C a|B|^{-1} \max \chi_{B}^{2}
$$

If we use that the Hilbert-Schmidt norm is greater than the operator norm, $\|Z\| \leq\|Z\|_{\mathrm{HS}}$, we find

$$
\|Z\| \leq|B|^{-1}\left(\iint \chi_{B}(x)^{2} W(x-y)^{2} \chi_{B}(y)^{2} \mathrm{~d} x \mathrm{~d} y\right)^{1 / 2} \leq|B|^{-1} \max W \int \chi_{B}^{2} \leq C a R^{-3} \max \chi_{B}^{2} .
$$

Combining the above lemma with $(71),(76),(77)$, and $(78)$ gives the following result.

LEMMA 4.4. For all $\sigma \in \mathbb{R}$ we have the following estimate

$$
H_{\mathrm{Quad}}+\sigma \mathcal{Q}_{1}^{\prime} \geq \frac{1}{2}(2 \pi)^{-3} \int_{\mathbb{R}^{3}} h_{\sigma}(k) \mathrm{d} k-C n_{+} a \min \left\{R^{-3},|B|^{-1}\right\} \max \chi_{B}^{2}
$$

on each $n$-particle sector (if $n=0$ then $h_{\sigma}(k)=0$ ), where

$$
\begin{aligned}
h_{\sigma}(k)= & n^{-1} \tau_{B}\left(k^{2}\right)\left(b_{k}^{*} b_{k}+b_{-k}^{*} b_{-k}\right)+\widehat{W}(k)|B|^{-1}\left(b_{k}^{*} b_{k}+b_{-k}^{*} b_{-k}+b_{k}^{*} b_{-k}^{*}+b_{k} b_{-k}\right) \\
& +\sigma(n-\rho|B|) \widehat{W}(k)|B|^{-3 / 2}\left(\widehat{\widehat{\chi}_{B}(k)}\left(b_{k}+b_{-k}^{*}\right)+\widehat{\chi}_{B}(k)\left(b_{k}^{*}+b_{-k}\right)\right) .
\end{aligned}
$$

Note that if $B$ is a large box, then the last term in 82 is small compared to the Neumann gap. The same is true if $B$ is a small box with smallest side length $\lambda_{1} \geq \rho^{-\frac{1}{3}}$, since then $C n_{+} a \min \left\{R^{-3},|B|^{-1}\right\} \max \chi_{B}^{2} \leq C n_{+} \rho a$, which, in view of Condition 3 below, is smaller than the Neumann gap on the small box.

We shall now give an estimate on $h_{\sigma}(k)$, which is based on a simple version of Bogolubov's 
treatment of quadratic Hamiltonians. This estimate requires, however, assumptions which will not be fulfilled in all our situations. The following result is Theorem 6.3 in [13] except that we state it here a bit more generally. In the original [13] it was required $\mathcal{A} \geq \mathcal{B}>0$, but this is not needed. The operators $b_{ \pm}$can, for example, be any commuting pair of bounded operators (the case we will use here) or they can be annihilation operators in Fock space (the original Bogolubov case).

THEOREM 4.5 (Simple case of Bogolubov's method).

For arbitrary $\mathcal{A}, \mathcal{B} \in \mathbb{R}$ satisfying $-\mathcal{A}<\mathcal{B} \leq \mathcal{A}$ and $\kappa \in \mathbb{C}$ we have the operator inequality

$$
\begin{gathered}
\mathcal{A}\left(b_{+}^{*} b_{+}+b_{-}^{*} b_{-}\right)+\mathcal{B}\left(b_{+}^{*} b_{-}^{*}+b_{+} b_{-}\right)+\kappa\left(b_{+}^{*}+b_{-}\right)+\bar{\kappa}\left(b_{+}+b_{-}^{*}\right) \\
\geq-\frac{1}{2}\left(\mathcal{A}-\sqrt{\mathcal{A}^{2}-\mathcal{B}^{2}}\right)\left(\left[b_{+}, b_{+}^{*}\right]+\left[b_{-}, b_{-}^{*}\right]\right)-\frac{2|\kappa|^{2}}{\mathcal{A}+\mathcal{B}}
\end{gathered}
$$

where $b_{ \pm}$are operators (possibly unbounded) on a Hilbert space satisfying $\left[b_{+}, b_{-}\right]=0$.

Proof. The proof is essentially the same as in the original [13]. See also [22].

When applying Theorem 4.5 with $\kappa=0$, we can replace $\mathcal{B}$ by $-\mathcal{B}$, even if $\mathcal{B}=\mathcal{A}$, without changing the lower bound. This is easily seen by replacing $b_{ \pm}$by $i b_{ \pm}$. Hence

$$
\left|\mathcal{B}\left(b_{+}^{*} b_{-}^{*}+b_{+} b_{-}\right)\right| \leq \mathcal{A}\left(b_{+}^{*} b_{+}+b_{-}^{*} b_{-}\right)+\frac{1}{2}\left(\mathcal{A}-\sqrt{\mathcal{A}^{2}-|\mathcal{B}|^{2}}\right)\left(\left[b_{+}, b_{+}^{*}\right]+\left[b_{-}, b_{-}^{*}\right]\right),
$$

which we will use on page 43 . When applying this theorem to estimate $h_{\sigma}(k)$, we will take $b_{+}=b_{k}, b_{-}=b_{-k}$, restricted to the appropriate $n$-particle sector,

$$
\mathcal{A}=n^{-1} \tau_{B}\left(k^{2}\right)+\widehat{W}(k)|B|^{-1}, \quad \mathcal{B}=\widehat{W}(k)|B|^{-1}, \quad \kappa=\sigma(n-\rho|B|) \widehat{W}(k)|B|^{-3 / 2} \widehat{\chi}_{B}(k) .
$$

This choice of $\mathcal{A}$ and $\mathcal{B}$ does not necessarily satisfy the conditions in Theorem 4.5. We will now give conditions for when these are satisfied. We first observe that $\widehat{W}(0)=\int W(x) \mathrm{d} x>0$ and thus

$$
\widehat{W}(k)=\int \cos (k x) W(x) \mathrm{d} x \geq \int\left(1-\frac{1}{2}(k x)^{2}\right) W(x) \mathrm{d} x>0
$$

if $|k|<R^{-1}$ (using that $W$ has the same range as $v_{R}$, i.e., $R$ ). Hence $\mathcal{B}>0$ for these values of $k$, and the conditions in the theorem are satisfied since $\tau_{B} \geq 0$.

To ensure the condition for $|k| \geq R^{-1}$, we will use that $R<\delta d s \ell$ by Condition 1 . We may then from the definitions (68) and (70) of $\tau_{B}$ assume that $\tau_{B}\left(k^{2}\right) \geq \frac{1}{2} k^{2}$ for $|k| \geq R^{-1} \geq$ 
$\delta^{-1}(d s \ell)^{-1}$. Given $C^{\prime}>0$ we have for these $k$, since $|\mathcal{B}|=|B|^{-1}|\widehat{W}(k)| \leq C a /|B|$, that

$$
\mathcal{A} \geq \frac{1}{2} n^{-1} R^{-2}-C a|B|^{-1}>C^{\prime} a|B|^{-1}
$$

if $n|B|^{-1} \leq c\left(a R^{2}\right)^{-1}$ for $c$ sufficiently small (depending only on $v_{1}$ and $C^{\prime}$ ). In particular, for $|k| \geq R^{-1}$ we then have $-\mathcal{A}<|\mathcal{B}| \leq \mathcal{A}$ and are therefore allowed to use Theorem 4.5 to bound $h_{\sigma}(k)$.

This implies that $\mathcal{A}$ is positive and that $\mathcal{A} \geq|\mathcal{B}|$. In this case we are therefore allowed to use Theorem 4.5 to bound $h_{\sigma}(k)$ and in fact we may assume that $\mathcal{A}+\mathcal{B} \geq 2|\mathcal{B}|$ if $c$ is sufficiently small. When the condition $n|B|^{-1} \leq c\left(a R^{2}\right)^{-1}$ can not be satisfied, which only happens on page 36 in Case 2 of the proof of Lemma 5.2 , we use 62 instead.

LEMMA 4.6. There exists $c>0$ (depending only on $v_{1}$ ) such that if $n|B|^{-1} \leq c\left(a R^{2}\right)^{-1}$ then we have on each $n$-particle sector (if $n=0$ then $h_{\sigma}(k)=0$ ) for all $k \in \mathbb{R}^{3}$

$$
\begin{aligned}
h_{\sigma}(k) \geq & -\left(n^{-1} \tau_{B}\left(k^{2}\right)+|B|^{-1} \widehat{W}(k)-\sqrt{n^{-2} \tau_{B}\left(k^{2}\right)^{2}+2 n^{-1}|B|^{-1} \tau_{B}\left(k^{2}\right) \widehat{W}(k)}\right) n_{0} \int \chi_{B}^{2} \\
& -\sigma^{2}(n-\rho|B|)^{2}|B|^{-2}\left|\widehat{\chi}_{B}(k)\right|^{2}|\widehat{W}(k)| .
\end{aligned}
$$

Proof. As we just saw, we can choose $c>0$ such that we may use Bogolubov's method from Theorem 4.5 with $\mathcal{A}+\mathcal{B} \geq 2|\mathcal{B}|$. This gives the estimate

$$
2|\kappa|^{2}(\mathcal{A}+\mathcal{B})^{-1} \leq \sigma^{2}(n-\rho|B|)^{2}|B|^{-2}\left|\widehat{\chi}_{B}(k)\right|^{2}|\widehat{W}(k)|
$$

Now 87 follows, since we have already seen in 75 that $\left[b_{k}, b_{k}^{*}\right] \leq n_{0} \int \chi_{B}^{2}$ and in 86 that $\widehat{W}(k)>0$ for $|k|<R^{-1}$.

We shall primarily use the above lemma with $\sigma=0$. On page 48 we will also use it with $\sigma=1$ on the large box. If we could replace $|\widehat{W}(k)|$ by $\widehat{W}(k)$ in the last term in (87) an integration over $k$ gives

$$
-\frac{1}{2}(2 \pi)^{-3} \sigma^{2}(n-\rho|B|)^{2}|B|^{-2} \int\left|\widehat{\chi}_{B}(k)\right|^{2} \widehat{W}(k) \mathrm{d} k=-\sigma^{2}(n-\rho|B|)^{2} \mathcal{U}_{B},
$$

using the notation (22), which then exactly cancels the first positive term in (57).

Now we estimate the error in approximating the integral over the last term in (87) by (88). We recall that $\widehat{W}(k)>0$ for $|k|<R^{-1}$ by 86 , that $|\widehat{W}(k)| \leq C a$ and that 
$\chi_{u}(x)=\chi\left(x \ell^{-1}-u\right)$. Then we use 34 to obtain the bound

$$
C \sigma^{2}|B|^{-1} a(n-\rho|B|)^{2}(R / \ell)^{2 M} \int_{|k| \geq R^{-1}}|k|^{2 M}|\widehat{\chi}(k)|^{2} \mathrm{~d} k \leq C \sigma^{2}|B|^{-1} a(n-\rho|B|)^{2}(R / \ell)^{2 M},
$$

where $M$ is the integer in the definition 13 of $\chi$. We have by Condition 1 that $R \ll \ell$ and this will be enough to control the last term in (87) if $M$ is sufficiently large.

\section{A Priori Bounds on the Non-Quadratic Part of the Hamiltonian and on $n$}

We shall eventually prove that the lowest energy of the box Hamiltonian $H_{B}$ will be achieved when the particle number $n$ is close in an appropriate sense to $\rho|B|$. In this subsection we will give a much weaker a priori bound on $n$. The main difficulty lies in treating the (possibly) rectangular small boxes $B\left(u, u^{\prime}\right)$ of side lengths $\lambda_{1} \leq \lambda_{2} \leq \lambda_{3} \leq d \ell$.

LEMMA 5.1 (Estimates on the non-quadratic part of $H_{B}$ ).

On each $n$-particle sector, if $B$ is a small box, we have

$$
H_{B}-H_{\mathrm{Quad}} \geq\left(1-\varepsilon_{0}\right) \varepsilon_{T} \widetilde{b}(d \ell)^{-2} n_{+}+\left[\frac{7}{8}|n-\rho| B \|^{2}-C|n-\rho| B|| n_{+}-C n-C n n_{+}\right] \mathcal{U}_{B}
$$

and, if $B$ is a large box,

$$
H_{B}-H_{\mathrm{Quad}} \geq\left(1-\varepsilon_{0}\right) b \ell^{-2} n_{+}+\left[\frac{7}{8}|n-\rho| B||^{2}-C|n-\rho| B|| n_{+}-C n-C n n_{+}\right] \mathcal{U}_{B} .
$$

Here $\widetilde{b}=\left(1+\pi^{-2}\right)^{-1}$ and $b$ is the universal constants appearing in 48.

Proof. We use Equation (57), Lemma 4.2 (with $\varepsilon_{1}^{\prime}=\frac{|n-\rho B|}{8 n}$ if $n \neq 0$ ), (63), 64), 65), the respective Neumann gaps and the fact that $n_{+} \leq n$ to obtain $(90)$ and $(91)$.

The constant $\frac{7}{8}$ is of course not optimal and has been chosen for notational purposes only. To prove the next lemma, we would like to use that $n_{+}$is much smaller than $n$. This follows from Lemma 4.1 in view of the following condition, which we henceforth assume to hold.

CONDITION 3: We require

$$
(\sqrt{\rho a} d \ell)^{2} \leq \delta \varepsilon_{T}, \quad \varepsilon_{T} \ln \left(\frac{d s \ell}{R}\right)<\delta d s \ell \sqrt{\rho a} \quad \text { and } \quad \varepsilon_{0} \frac{a}{R}<\delta \sqrt{\rho a^{3}}
$$


for $0<\delta<\delta_{\eta}<1$, where $\eta$ is as in Assumtion 2. This shows that we need $M \geq 13$.

LEMMA 5.2 (A priori bound on $n$ ).

There is a constant $C_{0}>0$ such that for any $n$-particle state on a small box satisfying $\left\langle\psi\left|H_{B}\right| \psi\right\rangle \leq 0$, we have

$$
n \leq C_{0}|B| \max \left\{\prod_{j=1}^{3}\left(\min \left\{\lambda_{j}, R\right\}\right)^{-1}, \rho\right\}=: K_{B}
$$

Proof. Assume $C_{0} \geq 2$. Then $K_{B} \geq C_{0}|B| \prod_{j=1}^{3} \lambda_{j}^{-1}=C_{0} \geq 2$. If $n \geq K_{B}$, let $m$ be the integer part of $n K_{B}^{-1}$. We can then divide the particles into $m$ groups of particles consisting of $n_{1}, \ldots, n_{m}$ particles where $\sum_{j=1}^{m} n_{j}=n$ and

$$
\frac{1}{2} K_{B} \leq n_{j} \leq 2 K_{B}, \quad j=1, \ldots, m
$$

We now use that the interaction $\omega_{B}$ between the particles is non-negative and thereby get the following lower bound if we ignore the interactions between the groups and correct for the background self-energy term

$\left\langle\psi\left|H_{B}\right| \psi\right\rangle-\rho^{2}|B|^{2} \mathcal{U}_{B} \geq m \inf \left\{\left\langle\psi^{\prime}\left|H_{B}\right| \psi^{\prime}\right\rangle-\rho^{2}|B|^{2} \mathcal{U}_{B} \mid \psi^{\prime}\right.$ has $n^{\prime}$ particles in $\left.B, \frac{1}{2} K_{B} \leq n^{\prime} \leq 2 K_{B}\right\}$

Our aim is to prove that if $C_{0}$ is large enough, then

$$
\left\langle\psi^{\prime}\left|H_{B}\right| \psi^{\prime}\right\rangle-\rho^{2}|B|^{2} \mathcal{U}_{B} \geq 0
$$

if $\psi^{\prime}$ has particle number $n^{\prime}$ satisfying $K_{B} / 2 \leq n^{\prime} \leq 2 K_{B}$. We have

$$
\rho^{2}|B|^{2} \leq C_{0}^{-2} K_{B}^{2} \leq 4 C_{0}^{-2} n^{\prime 2}
$$

Thus we have $\left|n^{\prime}-\rho\right| B|| \geq\left(1-\frac{2}{C_{0}}\right) n^{\prime}$ and, using that $n^{\prime} \geq \frac{C_{0}}{2}$, that $n^{\prime} \leq \frac{2}{C_{0}} n^{\prime 2}$. With $n_{+}^{\prime}=\left\langle\psi^{\prime}\left|n_{+}\right| \psi^{\prime}\right\rangle$ we obtain from Lemma 4.1. Condition 3, Lemma 5.1 and 95) that

$$
\left\langle\psi^{\prime}\left|H_{B}-H_{\mathrm{Quad}}\right| \psi^{\prime}\right\rangle-\rho^{2}|B|^{2} \mathcal{U}_{B} \geq\left(1-\varepsilon_{0}\right) \varepsilon_{T}\left(1+\pi^{-2}\right)^{-1}(d \ell)^{-2} n_{+}^{\prime}+\frac{3}{4} n^{\prime 2} \mathcal{U}_{B}
$$

if $C_{0}$ is sufficiently large and $\delta$ sufficiently small. It remains to bound $H_{\text {Quad }}$. To do this, we differentiate between whether or not we are allowed to apply Bogolubov's method. With $c>0$ being the constant in Lemma 4.6 which will allow us to use the Bogolubov bound, we first treat boxes where the side length and the parameter $R$ are not too small in the following 
sense.

Case I: $K_{B}<(c / 2)|B|\left(a R^{2}\right)^{-1}$

Here we are allowed to use Bogolubov's method, i.e., Lemma 4.4 and Lemma 4.6. Combining A.7), A.8), A.9 and A.16), we get

$$
\begin{aligned}
\left\langle\psi^{\prime}\left|H_{\mathrm{Quad}}\right| \psi^{\prime}\right\rangle & \geq \frac{1}{2}(2 \pi)^{-3} \int_{\mathbb{R}^{3}} h_{0}(k) \mathrm{d} k-C n_{+}^{\prime} a \min \left\{R^{-3},|B|^{-1}\right\} \max \chi_{B}^{2} \\
& \geq-C n^{\prime} a(d s \ell)^{-3} \max \chi_{B}^{2}-C n^{\prime} \frac{n^{\prime}}{|B|} a \frac{a}{R} \max \chi_{B}^{2}-C n^{\prime} \frac{n^{\prime 2}}{|B|^{2}} a^{3} R \max \chi_{B}^{2}-C n^{\prime} \frac{a}{R^{3}} \max \chi_{B}^{2} \\
& \geq-C n^{\prime} \frac{a}{R^{3}} \max \chi_{B}^{2}-C n^{\prime} \frac{K_{B}}{|B|} a \frac{a}{R} \max \chi_{B}^{2}-C n^{\prime} \frac{K_{B}^{2}}{|B|^{2}} a^{3} R \max \chi_{B}^{2} \\
& \geq-C n^{\prime} \frac{a}{R^{3}} \max \chi_{B}^{2}
\end{aligned}
$$

Combining (94) and (96) and noting that 25) implies $\frac{a}{R^{3}} \max \chi_{B}^{2} \mathcal{U}_{B}^{-1} \leq \frac{C}{C_{0}} K_{B}$ gives

$$
\begin{aligned}
\left\langle\psi^{\prime}\left|H_{B}\right| \psi^{\prime}\right\rangle-\rho^{2}|B|^{2} \mathcal{U}_{B} & \geq C n^{\prime 2} \mathcal{U}_{B}-C n^{\prime} \frac{a}{R^{3}} \max \chi_{B}^{2} \\
& =C n^{\prime} \mathcal{U}_{B}\left(n^{\prime}-C \mathcal{U}_{B}^{-1} \frac{a}{R^{3}} \max \chi_{B}^{2}\right) \\
& \geq C n^{\prime} \mathcal{U}_{B}\left(n^{\prime}-C C_{0}^{-1} n^{\prime}\right)
\end{aligned}
$$

which is positive if $C_{0}$ is sufficiently large.

Case II: $K_{B} \geq(c / 2)|B|\left(a R^{2}\right)^{-1}$

By Condition 1 and the Case II assumption we have

$$
\frac{K_{B}}{|B|}=C_{0} \prod_{j=1}^{3}\left(\min \left\{\lambda_{j}, R\right\}\right)^{-1} \geq \frac{c}{2}\left(a R^{2}\right)^{-1}
$$

Since $R, \lambda_{j} \leq d \ell$ we have $|B| \leq C a(d \ell)^{2}$ such that $\max \chi_{B}^{2} \leq C\left(\frac{a}{d \ell}\right)^{4(M+1)}$ by 16). By Lemma 4.1 and Condition 3 , we have $n_{+} \leq C \delta n \max \chi_{B}^{2}$. From the lower bound in 25) we get

$$
\begin{aligned}
C n_{+}^{\prime 2} \frac{a}{R^{3}} \max \chi_{B}^{2} & \leq C \delta n^{\prime 2} \frac{a}{R^{3}} \max \chi_{B}^{4} \\
& \leq C \delta n^{\prime 2}|B| \prod_{j=1}^{3}\left(\min \left\{\lambda_{j}, R\right\}\right)^{-1} \max \chi_{B}^{2} \mathcal{U}_{B} \\
& \leq C \delta n^{\prime 2}\left(\frac{d \ell}{R}\right)^{3}\left(\frac{a}{d \ell}\right)^{4(M+1)} \mathcal{U}_{B}
\end{aligned}
$$


Together with equation 96 and the estimate

$$
\left\langle\psi^{\prime}\left|H_{\mathrm{Quad}}\right| \psi^{\prime}\right\rangle \geq\left\langle\psi^{\prime}\left|\mathcal{Q}_{2}^{\prime}\right| \psi^{\prime}\right\rangle \geq-\frac{5}{8} n^{\prime 2} \mathcal{U}_{B}-C n_{+}^{2} \frac{a}{R^{3}} \max \chi_{B}^{2},
$$

which follows from 62 , this yields

$$
\left\langle\psi^{\prime}\left|H_{B}\right| \psi^{\prime}\right\rangle-\rho^{2}|B|^{2} \mathcal{U}_{B} \geq \frac{1}{8} n^{\prime 2} \mathcal{U}_{B}-C n_{+}^{\prime 2} a R^{-3} \max \chi_{B}^{2} \geq 0
$$

provided $\delta$ is sufficiently small.

When applying the above lemma, we will assume that the box $B=B\left(u, u^{\prime}\right)$ has either smallest side length $\lambda_{1} \leq \rho^{-\frac{1}{3}}$ or $\lambda_{1}>\rho^{-\frac{1}{3}}$. Note that if $\lambda_{1}>\rho^{-\frac{1}{3}}$, we get $n \leq$ $C_{0}|B| \max \left\{R^{-3}, \rho\right\}$ and may apply Lemma 4.6 .

\subsection{A Priori Bounds on the Energy in the Small Box}

Small boxes at the boundary of the large box may be arbitrarily small. We first consider the case of boxes which are so small that Bogolubov's method can not be applied. By the lemma below these boxes only contribute to $e_{0}(\rho)$ by an amount, which is of higher order than the LHY-term.

LEMMA 5.3 (Lower bound on the energy on small boxes with $\lambda_{1} \leq \rho^{-\frac{1}{3}}$ ).

For any $n$-particle state $\psi$ on a small box $B$ with smallest side length $\lambda_{1} \leq \rho^{-\frac{1}{3}}$, we have

$$
\left\langle\psi\left|H_{B}\right| \psi\right\rangle \geq-C|B| \max \left\{\rho, R^{-3}\right\} \frac{a}{R^{3}}\left(\frac{\rho^{-\frac{1}{3}}}{d \ell}\right)^{4 M+2}
$$

where $M$ is the integer in the definition (13) of $\chi$. For all $u \in \mathbb{R}^{3}$

$$
\int_{\lambda_{1}\left(B\left(u, u^{\prime}\right)\right) \leq \rho^{-\frac{1}{3}}} H_{u u^{\prime}} \mathrm{d} u^{\prime} \geq-C \mathcal{L},
$$

with $\mathcal{L}=|B| \max \left\{\rho, R^{-3}\right\} \frac{a}{R^{3}}\left(\frac{\rho^{-\frac{1}{3}}}{\ell}\right)^{2 M}$.

Proof. We use Lemma 4.1 to get the bound $\left\langle H_{B}\right\rangle \geq-C n \rho|B| \mathcal{U}_{B}$. Since we may assume that $\left\langle H_{B}\right\rangle \leq 0$, we use Lemma 5.2 , which together with $\lambda_{1} \leq \rho^{-\frac{1}{3}}$ gives $n \leq C|B|\left(\min \left\{\lambda_{1}, R\right\}\right)^{-3}$. Using the upper bound in (25) followed by (16), we arrive at

$$
\left\langle H_{B}\right\rangle \geq-C \frac{|B|^{2}}{\min \left\{\lambda_{1}^{3}, R^{3}\right\}} \rho \frac{a}{R^{3}} \max \chi_{B}^{2}
$$




$$
\geq-C \frac{\lambda_{1}(d \ell)^{2}|B|}{\min \left\{\lambda_{1}^{3}, R^{3}\right\}} \rho \frac{a}{R^{3}}\left(\frac{\lambda_{1}}{\ell}\right)^{2(M+1)} .
$$

The estimate in (100) is obtained by integrating over $u^{\prime}$ such that $B\left(u, u^{\prime}\right)$ has $\lambda_{1} \leq \rho^{-\frac{1}{3}}$, which gives a volume smaller than $C d^{-2} \frac{\rho^{-\frac{1}{3}}}{d \ell}$, and using that $\lambda_{1} \leq \rho^{-\frac{1}{3}}<d \ell$.

Now we turn to the case of small boxes which have smallest side length larger than $\rho^{-\frac{1}{3}}$ and where Bogolubov's method, i.e., Lemma 4.6, therefore is applicable.

LEMMA 5.4 ( $2^{\text {nd }}$ a priori bound on $n$ for small boxes with $\left.\lambda_{1} \geq \rho^{-\frac{1}{3}}\right)$. If $B$ is a small box with $\lambda_{1} \geq \rho^{-\frac{1}{3}}$, then there exists a constant $C_{1}>1$ such that for any $n$-particle state satisfying $\left\langle\psi\left|H_{B}\right| \psi\right\rangle \leq 0$ we have

$$
n \leq C_{1} \rho|B|
$$

Proof. We may assume that $R \leq \rho^{-\frac{1}{3}}$, since otherwise the lemma follows from the first a priori bound on $n$ in Lemma 5.2 . Hence the estimates (25) and (26) give

$$
C^{-1} \frac{a}{|B|} \max \chi_{B}^{2} \leq \mathcal{U}_{B} \leq C \frac{a}{|B|} \max \chi_{B}^{2} .
$$

Assume $n \geq C_{1} \rho|B|$ with $C_{1}>1$. Note here that $\rho|B| \geq \rho \rho^{-1}=1$ since $\lambda_{1} \geq \rho^{-\frac{1}{3}}$ and we therefore by assumption have that $n \geq C_{1}$. Now Lemma 4.4. Lemma 5.1 and 102 imply

$$
\begin{aligned}
\left\langle H_{B}\right\rangle & \geq C n^{2} \mathcal{U}_{B}+\frac{1}{2}(2 \pi)^{-3} \int h_{0}(k) \mathrm{d} k-C n_{+} \frac{a}{|B|} \max \chi_{B}^{2} \\
& \geq C n^{2} \frac{a}{|B|} \max \chi_{B}^{2}+\frac{1}{2}(2 \pi)^{-3} \int h_{0}(k) \mathrm{d} k,
\end{aligned}
$$

provided $C_{1}$ is sufficiently large and $\delta$ is sufficiently small. Combining A.7, A.8, A.9 and A.16 in the appendix, we get

$$
\int h_{0}(k) \mathrm{d} k \geq-C \frac{n}{|B|} a(d s \ell)^{-3} \int \chi_{B}^{2}-C \frac{n^{2}}{|B|^{2}} \frac{a^{2}}{R} \int \chi_{B}^{2}-C \frac{n^{3} a^{3}}{|B|^{3}} R \int \chi_{B}^{2} .
$$

Since $n \leq C_{0}|B| R^{-3}$ by Lemma 5.2 the assumption $n \geq C_{1} \rho|B|$ leads to

$$
0 \geq\left\langle H_{B}\right\rangle \geq\left(C n^{2}-C n^{2} \rho^{-1}(d s \ell)^{-3}-C n^{2} \frac{a}{R}-C n^{2} \frac{a^{2}}{R^{2}}\right) \frac{a}{|B|} \max \chi_{B}^{2},
$$

which contradicts that $C-C\left(\frac{\rho^{-\frac{1}{3}}}{d s \ell}\right)^{3}-C \frac{a}{R}-C \frac{a^{2}}{R^{2}}>0$ by Condition 1 if $\delta$ is sufficiently small. 
LEMMA 5.5 (Lower bound on the energy on small boxes with $\lambda_{1} \geq \rho^{-\frac{1}{3}}$ ). If $B$ is a small box with $\lambda_{1} \geq \rho^{-\frac{1}{3}}$ and $\psi$ is an n-particle state satisfying $\left\langle\psi\left|H_{B}\right| \psi\right\rangle \leq 0$, then

$$
\begin{aligned}
\left\langle H_{B}\right\rangle \geq & C \varepsilon_{T}(d \ell)^{-2} n_{+}+\frac{3}{4}|n-\rho| B||^{2} \mathcal{U}_{B}-C \rho a \\
& -\frac{1}{4}(2 \pi)^{-3} \rho^{2} \frac{1}{R} \int \frac{\widehat{v}_{1}(k)^{2}}{|k|^{2}} \mathrm{~d} k \int \chi_{B}^{2}-C \rho^{2} a\left(\rho a^{3}\right)^{\frac{1}{2}} \int \chi_{B}^{2}(\sqrt{\rho a} d s \ell)^{-3} .
\end{aligned}
$$

Proof. We start by estimating $H_{B}-H_{\text {Quad. }}$. Recall that $n \leq C \rho|B|$ by Lemma 5.4 and $\mathcal{U}_{B} \leq C \frac{a}{|B|}$ by $(26)$. Now Lemma 5.1 and that that $(d \ell \sqrt{\rho a})^{2}<\delta \varepsilon_{T}$ by Condition 3 yields

$$
\begin{aligned}
H_{B}-H_{\mathrm{Quad}} & \geq C \varepsilon_{T}(d \ell)^{-2} n_{+}+\left.\frac{7}{8}|n-\rho| B\right|^{2} \mathcal{U}_{B}-C \rho a-C \rho a n_{+} \\
& \geq C \varepsilon_{T}(d \ell)^{-2} n_{+}+\left.\frac{7}{8}|n-\rho| B\right|^{2} \mathcal{U}_{B}-C \rho a .
\end{aligned}
$$

We use Lemma 4.4 with $\sigma=0$ to estimate the quadratic part

$$
H_{\mathrm{Quad}} \geq \frac{1}{2}(2 \pi)^{-3} \int_{\mathbb{R}^{3}} h_{0}(k) \mathrm{d} k-C n_{+} a \min \left\{R^{-3},|B|^{-1}\right\} \max \chi_{B}^{2} .
$$

Using the bounds A.7, A.8, A.9 and A.16 in the appendix, we obtain

$$
\begin{aligned}
\frac{1}{2}(2 \pi)^{-3} \int h_{0}(k) \mathrm{d} k \geq & -\frac{1}{4}(2 \pi)^{-3}\left(1+C \varepsilon_{0}\right) \frac{1}{R} \int\left(\frac{n}{|B|}\right)^{2} \frac{\widehat{v}_{1}(k)^{2}}{|k|^{2}} \mathrm{~d} k \int \chi_{B}^{2} \\
& -C \rho^{2} a \frac{a}{d s \ell} \ln \left(\frac{d s \ell}{R}\right) \int \chi_{B}^{2}-C(\rho a)^{3} R \int \chi_{B}^{2} \\
& -C \rho a(d s \ell)^{-3} \int \chi_{B}^{2} .
\end{aligned}
$$

The second term in 107$)$ is smaller than $C n_{+} \rho a$, since we assumed that $\lambda_{1} \geq \rho^{-\frac{1}{3}}$, and may therefore also be absorbed into the Neumann gap. Instead of estimating the term $|n-\rho| B||$, we can use that if $C_{1}>0$, then

$$
\begin{aligned}
& -\frac{1}{4}(2 \pi)^{-3}\left(\frac{n}{|B|}\right)^{2} \frac{1}{R} \int \frac{\widehat{v}_{1}(k)^{2}}{|k|^{2}} \mathrm{~d} k \int \chi_{B}^{2}+C_{1}|n-\rho| B \|^{2} \mathcal{U}_{B} \\
\geq & -\frac{1}{4}(2 \pi)^{-3} \rho^{2} \frac{1}{R} \int \frac{\widehat{v}_{1}(k)^{2}}{|k|^{2}} \mathrm{~d} k \int \chi_{B}^{2}-C \rho^{2} a \frac{a^{2}}{R^{2}} \max \left\{\rho R^{3}, 1\right\} \int \chi_{B}^{2} .
\end{aligned}
$$


To see this we insert the following estimate, which follows from (25),

$$
\mathcal{U}_{B} \geq C \min \left\{\frac{\rho^{-1}}{R^{2}}, R\right\} \frac{1}{R} \frac{a}{|B|} \max \chi_{B}^{2}
$$

The $n^{2}$-term in 109 is then positive if $\delta$ is sufficiently small and we can verify (110) by optimizing over $n$ and noticing that the optimal particle number satisfies $n \leq \rho|B|(1+$ $\left.C \frac{a}{R} \max \left\{\rho R^{3}, 1\right\}\right)$. After noting that by Condition 1 and Condition 3

$$
(\sqrt{\rho a} d s \ell)^{-3} \geq \varepsilon_{0}\left(\rho a^{3}\right)^{-\frac{1}{2}} \frac{a}{R}+(\sqrt{\rho a} d s \ell)^{-1} \ln \left(\frac{d s \ell}{R}\right)+\sqrt{\rho a} R+\left(\rho a^{3}\right)^{-\frac{1}{2}} \frac{a^{2}}{R^{2}} \max \left\{\rho R^{3}, 1\right\}
$$

the lemma follows.

\section{Estimates on the Large Box}

From now on we will only focus on the large box, where we have $\int \chi_{B}^{2}=|B|=\ell^{3}$. The following lemma gives a lower bound for the operator $H_{B}$ on the large box and is the starting point for the bounds on $n_{+}$and $|n-\rho| B||$ on the large box.

LEMMA 6.1. On a large box we have

$$
H_{B} \geq-\frac{1}{4}(2 \pi)^{-3} \rho^{2} \frac{1}{R} \int \frac{\widehat{v}_{1}(k)^{2}}{|k|^{2}} \mathrm{~d} k|B|+C b \ell^{-2} n_{+}-C \rho^{2} a|B| \sqrt{\rho a^{3}}(\sqrt{\rho a} d s \ell)^{-3} .
$$

Proof. We use Lemma 3.10, together with (15), and sum the contribution of the small boxes. We use Lemma 5.5 for boxes with $\lambda_{1}>\rho^{-\frac{1}{3}}$, of which we have less then $C d^{-3}$. For the small boxes boxes with $\lambda_{1} \leq \rho^{-\frac{1}{3}}$ we use Lemma 5.3 and note that $\frac{\mathcal{L}}{|B|} \leq(\rho a)^{\frac{5}{2}}$ by Condition 1 if $M \geq 3$.

States satisfying the condition below will play an important role in our following estimates.

CONDITION 4: We require that that $B$ is a large box and that $\psi$ is a state with fixed particle number, which satisfies

$$
\left\langle\psi, H_{B} \psi\right\rangle \leq-\frac{1}{4}(2 \pi)^{-3} \rho^{2} \frac{1}{R} \int \frac{\widehat{v}_{1}(k)^{2}}{|k|^{2}} \mathrm{~d} k|B|+C \rho^{2} a|B| \sqrt{\rho a^{3}}(\sqrt{\rho a} d s \ell)^{-3} .
$$

LEMMA 6.2 (Control of $\left\langle n_{+}\right\rangle$in the large box). Assume that $\psi$ satisfies Condition \& then 
we have, with $\mathcal{S}:=\rho a \ell^{2}(\sqrt{\rho a} d s \ell)^{-3}$,

$$
\left\langle\psi, n_{+} \psi\right\rangle \leq C \rho|B| \sqrt{\rho a^{3}} \mathcal{S}
$$

Proof. Simply apply Lemma 6.1.

Note that Condition 5 below in particular ensures that the second term in (112), while being larger than the LHY-order, is of higher order than the leading order term. We therefore have that $\left\langle\psi, H_{B} \psi\right\rangle \leq 0$ if the requirement in Lemma 6.2 is satisfied and $\delta$ is sufficiently small. In this section we will introduce new error terms and these will be smaller than the LHY-order.

CONDITION 5: We require, with $\mathcal{S}$ as in Lemma 6.2.

$$
\sqrt{\rho a^{3}} \mathcal{S}<\delta \frac{a}{R}
$$

LEMMA 6.3. Assume that $\psi$ satisfies Condition 4. Then $n \leq C \rho|B|$ and

$$
\left\langle\psi, H_{\mathrm{Quad}} \psi\right\rangle \geq-\frac{1}{4}(2 \pi)^{-3}\left(\frac{n}{|B|}\right)^{2} \frac{1}{R} \int \frac{\widehat{v}_{1}(k)^{2}}{|k|^{2}} \mathrm{~d} k|B|-C \rho^{2} a \sqrt{\rho a^{3}}|B| \mathcal{E}_{\mathrm{Quad}}
$$

where

$$
\begin{aligned}
\mathcal{E}_{\mathrm{Quad}}= & (\sqrt{\rho a} s \ell)^{-3}+\left(\rho a^{3}\right)^{\frac{1}{2}} \frac{R}{a}+\varepsilon_{0}\left(\rho a^{3}\right)^{-\frac{1}{2}} \frac{a}{R} \\
& +(\sqrt{\rho a} s \ell)^{-1} \ln \left(\frac{s \ell}{R}\right)+\varepsilon_{T}(\sqrt{\rho a} d s \ell)^{-1} \ln \left(\frac{d s \ell}{R}\right) .
\end{aligned}
$$

Proof. We start with the bound on $n$ and assume that $n \geq C \rho|B|$. From Lemma 4.4. Lemma 4.6, A.2, A.17) and A.23) we obtain (using $n_{+} \leq C n(s \ell)^{-3}|B|$ by Lemma 6.2 and Condition 1 )

$$
\left\langle\psi, H_{\mathrm{Quad}} \psi\right\rangle \geq-C n^{2} \frac{a}{R} \frac{a}{|B|}-C n(s \ell)^{-3}|B| \frac{a}{|B|}
$$

For $\delta$ sufficiently small we have $\left\langle\psi, H_{B} \psi\right\rangle \leq 0$ by Condition 5 and that $n_{+} \leq C \sqrt{\rho a^{3}} \mathcal{S} n<$ $C \delta \frac{a}{R} n$ by Lemma 6.2. It then follows from Lemma 5.1 that $\left\langle\psi,\left(H_{B}-H_{\text {Quad }}\right) \psi\right\rangle \geq C n^{2} \frac{a}{|B|}$ if $\delta$ is sufficiently small and we obtain the contradiction

$$
0 \geq\left\langle\psi, H_{B} \psi\right\rangle \geq\left(C n^{2}-C n^{2} \frac{a}{R}-C n^{2}(s \ell)^{-3} \rho^{-1}\right) \frac{a}{|B|}
$$

Hence $n \leq C \rho|B|$. Applying A.17 to A.19 and A.23 we obtain the lower bound on $\left\langle\psi, H_{\mathrm{Quad}} \psi\right\rangle$. 
Clearly $\mathcal{E}_{\text {Quad }}<\mathcal{S}$ and, in contrast to the estimate on the small box, once we choose our parameters as in Lemma 7.1 we will have $\mathcal{E}_{\text {Quad }} \ll 1$.

LEMMA 6.4 (Improved bound on $n$ on the large box).

Assume that $\psi$ is an n-particle state which satisfies Condition 4. Then

$$
|n-\rho| B|| \leq C \rho|B|\left(\rho a^{3}\right)^{\frac{1}{4}} \mathcal{S}^{\frac{1}{2}}
$$

Proof. We use the bound on the non-quadratic part in Lemma 5.1 and the bound on $H_{\text {Quad }}$ in Lemma 6.3, the bound on $n_{+}$in Lemma 6.2. Then we use $\mathcal{E}_{\text {Quad }}<\mathcal{S}$ and, analogously to (110), a part of the positive term $|n-\rho| B||$ to control the integral corresponding to the second Born term and obtain

$$
\begin{aligned}
\left\langle\psi, H_{B} \psi\right\rangle \geq & C \ell^{-2} n_{+}+\left[\left.\frac{7}{8}|n-\rho| B\right|^{2}-C|n-\rho| B|| n_{+}-C n-C n n_{+}\right] \mathcal{U}_{B} \\
& -\frac{1}{4}(2 \pi)^{-3}\left(\frac{n}{|B|}\right)^{2} \frac{1}{R} \int \frac{\widehat{v}_{1}(k)^{2}}{|k|^{2}} \mathrm{~d} k|B|-C \rho^{2} a \sqrt{\rho a^{3}}|B| \mathcal{E}_{\text {Quad }} \\
\geq & {\left[\left.C|n-\rho| B\right|^{2}-C \rho^{2}|B|^{2} \sqrt{\rho a^{3}} \mathcal{S}\right] \frac{a}{|B|} } \\
& -\frac{1}{4}(2 \pi)^{-3} \rho^{2} \frac{1}{R} \int \frac{\widehat{v}_{1}(k)^{2}}{|k|^{2}} \mathrm{~d} k|B|-C \rho^{2} a \frac{a^{2}}{R^{2}}|B| .
\end{aligned}
$$

Hence $|n-\rho| B|| \leq C \rho|B|\left(\rho a^{3}\right)^{\frac{1}{4}} \mathcal{S}^{\frac{1}{2}}$, by Condition 1 .

We will apply the following theorem, whose proof can be found in [13].

THEOREM 6.5 (Localization of large matrices).

Suppose that $\mathcal{A}$ is an $(N+1) \times(N+1)$ Hermitean matrix and let $\mathcal{A}^{k}$, with $k=0,1, \ldots, N$, denote the matrix consisting of the $k^{\text {th }}$ supra- and infra-diagonal of $\mathcal{A}$. Let $\psi \in \mathbb{C}^{N+1}$ be a normalized vector and set $d_{k}=\left\langle\psi, \mathcal{A}^{k} \psi\right\rangle$ and $\lambda=\langle\psi, \mathcal{A} \psi\rangle=\sum_{k=0}^{N} d_{k}$ ( $\psi$ need not be an eigenvector of $\mathcal{A})$. Choose some positive integer $\mathcal{M} \leq N+1$. Then, with $\mathcal{M}$ fixed, there is some $n \in[0, N+1-\mathcal{M}]$ and some normalized vector $\phi \in \mathbb{C}^{N+1}$ with the property that $\phi_{j}=0$ unless $n+1 \leq j \leq n+\mathcal{M}$ (i.e., $\phi$ has length $\mathcal{M}$ ) and such that

$$
\langle\phi, \mathcal{A} \phi\rangle \leq \lambda+\frac{C}{\mathcal{M}^{2}} \sum_{k=1}^{\mathcal{M}-1} k^{2}\left|d_{k}\right|+C \sum_{k=\mathcal{M}}^{N}\left|d_{k}\right|
$$

where $C>0$ is a universal constant. (Note that the first sum starts at $k=1$.)

We apply the theorem in the following way. Let $\Psi$ be a (normalized) $n$-particle wave function. Since the $n$-particle sector of Fock space is spanned by $n_{+}$-eigenfunctions, we can 
write $\Psi=\sum_{m=0}^{n} c_{m} \Psi_{m}$, with $\Psi_{m}$ normalized and $n_{+} \Psi_{m}=m \Psi_{m}$ for $m \in\{0,1, \ldots, n\}$. Since we aim to show a lower bound on the ground state energy density it is natural, in view of Theorem 2.1 and Theorem 3.10, to assume that

$$
\begin{aligned}
\left\langle\Psi,\left(-\varepsilon_{0} \Delta_{u}^{\mathcal{N}}+H_{B}\right) \Psi\right\rangle & \leq-\frac{1}{4}(2 \pi)^{-3} \rho^{2} \frac{1}{R} \int \frac{\widehat{v}_{1}(k)^{2}}{|k|^{2}} \mathrm{~d} k|B|+4 \pi \rho^{2} a|B| \sqrt{\rho a^{3}} \frac{128}{15 \sqrt{\pi}} \\
& =4 \pi \rho^{2}\left(a_{2}+a \sqrt{\rho a^{3}} \frac{128}{15 \sqrt{\pi}}\right)|B| .
\end{aligned}
$$

This lets us consider the $(n+1) \times(n+1)$ Hermitean matrix $\mathcal{A}_{m, m^{\prime}}=\left\langle\Psi_{m},\left(-\varepsilon_{0} \Delta_{u}^{\mathcal{N}}+H_{B}\right) \Psi_{m^{\prime}}\right\rangle$ and the vector $\psi=\left(c_{0}, c_{1}, \ldots, c_{n}\right)$. From the form of $H_{B}$ we obtain that $d_{k}=0$ for $k \geq 3$ and thus Theorem 6.5 yields a (normalized) state, $\widetilde{\psi}$, which, for given $\mathcal{M}$, has $n_{+}$-eigenvalues localized to an interval of length $\mathcal{M}$ and an energy that satisfies the bound

$$
\left\langle\Psi,\left(-\varepsilon_{0} \Delta_{u}^{\mathcal{N}}+H_{B}\right) \Psi\right\rangle \geq\left\langle\widetilde{\psi},\left(-\varepsilon_{0} \Delta_{u}^{\mathcal{N}}+H_{B}\right) \widetilde{\psi}\right\rangle-C \mathcal{M}^{-2}\left(\left|d_{1}\right|+\left|d_{2}\right|\right),
$$

where

$$
d_{1}:=\left\langle\Psi,\left(\mathcal{Q}_{1}^{\prime}+\mathcal{Q}_{1}^{\prime \prime}+\mathcal{Q}_{3}\right) \Psi\right\rangle \quad \text { and } \quad d_{2}:=\left\langle\Psi, \sum_{i<j}\left(Q_{i} Q_{j} w_{B}\left(x_{i}, x_{j}\right) P_{j} P_{i}+\text { h.c. }\right) \Psi\right\rangle .
$$

Under the assumption in (117) we will estimate $\left|d_{1}\right|$ and $\left|d_{2}\right|$ in Lemma 6.6. Adequate bounds on $\mathcal{M}$ are collected in Condition 6 below and properties of $\widetilde{\psi}$ are listed in Theorem 6.7. We will spend the rest of this section on establishing tools for obtaining a lower bound for $\left\langle\widetilde{\psi},\left(-\varepsilon_{0} \Delta_{u}^{\mathcal{N}}+H_{B}\right) \widetilde{\psi}\right\rangle$, which will be applied in the next section in our proof of Theorem 2.1

LEMMA 6.6 (Control of $d_{1}$ and $d_{2}$ ).

Let $d_{1}, d_{2}$ as in (119). If $B$ is a large box and $\Psi$ an $n$-particle state satisfying (117), then

$$
\left|d_{1}\right|+\left|d_{2}\right| \leq C \rho^{2} a|B| \frac{a}{R}
$$

Proof. First we estimate $\left|d_{1}\right|$. From Lemma 4.2, equation (64), which could have been stated as two-sided bounds, and that $n \leq C \rho|B|$, we obtain by setting $\varepsilon_{1}^{\prime}=c \frac{|n-\rho| B||}{\rho|B|}$ with $c$ sufficiently small and $\varepsilon_{1}^{\prime \prime}=1$

$$
\left|d_{1}\right| \leq(n-\rho|B|)^{2} \frac{a}{|B|}+C\left\langle\Psi\left|\rho a\left(\left(1+\varepsilon_{3}^{-1}\right) n_{+}+1\right)\right| \Psi\right\rangle+\varepsilon_{3}\left\langle\Psi\left|\sum_{i<j} Q_{j} Q_{i} w_{B}\left(x_{i}, x_{j}\right) Q_{i} Q_{j}\right| \Psi\right\rangle .
$$


We can use Lemmas 6.2 and 6.4 together with Conditions 1 and 5 to see that

$$
(n-\rho|B|)^{2} \frac{a}{|B|}+C\left\langle\Psi\left|\rho a\left(\left(1+\varepsilon_{3}^{-1}\right) n_{+}+1\right)\right| \Psi\right\rangle \leq C \rho^{2}|B| a \frac{a}{R}
$$

as long as we choose $\varepsilon_{3}$ to be a constant. Further we have

$$
\left\langle\Psi\left|\sum_{i<j} Q_{j} Q_{i} w_{B}\left(x_{i}, x_{j}\right) Q_{i} Q_{j}\right| \Psi\right\rangle \leq C \rho^{2} a|B| \frac{a}{R}
$$

since otherwise we get by combining Lemma 6.2 and Condition 5 with the estimates in the proof of Lemma 5.1

$$
\left\langle\Psi\left|H_{B}-H_{\mathrm{Quad}}\right| \Psi\right\rangle \geq\left. C|n-\rho| B\right|^{2} \frac{a}{|B|}+C \rho^{2} a|B| \frac{a}{R}
$$

which would contradict (117) in view of Lemma 6.3. It follows that $\left|d_{1}\right| \leq C \rho^{2} a|B| \frac{a}{R}$.

Now we estimate $\left|d_{2}\right|$. Note that $d_{2}$ corresponds to the terms containing $b^{*} b^{*}$ and $b b$ appearing in the second quantization of $\mathcal{Q}_{2}^{\prime}$ in 777 . Since $\mathcal{Q}_{2}^{\prime}$ is part of $H_{\text {Quad }}$ we utilize equation (84) with $\mathcal{A}$ and $\mathcal{B}$ as in 85 to bound $\left|d_{2}\right|=\left|\left\langle\Psi\left|\mathcal{B}\left(b_{k}^{*} b_{-k}^{*}+b_{k} b_{-k}\right)\right| \Psi\right\rangle\right|$. The contribution from the the commutator terms in (84) is controlled using (75). We have already seen in the proof of Theorem 6.3 that

$$
\begin{aligned}
& \left\langle\Psi\left|\frac{1}{2}(2 \pi)^{-3} \int\left(n^{-1} \tau_{B}\left(k^{2}\right)+|B|^{-1} \widehat{W}(k)-\sqrt{n^{-2} \tau_{B}\left(k^{2}\right)+2 n^{-1}|B|^{-1} \tau_{B}\left(k^{2}\right) \widehat{W}(k)}\right) n_{0} \int \chi_{B}^{2}\right| \Psi\right\rangle \\
& \leq C \rho^{2} a|B| \frac{a}{R} .
\end{aligned}
$$

It is therefore left to show that

$$
\left\langle\Psi\left|\frac{1}{2}(2 \pi)^{-3} n^{-1} \int \tau_{B}\left(k^{2}\right) b_{k}^{*} b_{k} \mathrm{~d} k\right| \Psi\right\rangle \leq C \rho^{2} a|B| \frac{a}{R}
$$

and that

$$
|B|^{-1}\left\langle\Psi\left|\frac{1}{2}(2 \pi)^{-3} \int \widehat{W}_{B}(k) b_{k}^{*} b_{k} \mathrm{~d} k\right| \Psi\right\rangle \leq C \rho^{2} a|B| \frac{a}{R}
$$

We show 123 first. As on page 29 we may assume that $n_{+} \geq 1$. From 79 and Lemma 4.3 we then see that

$$
|B|^{-1}\left\langle\Psi\left|\int \widehat{W}_{B}(k) b_{k}^{*} b_{k} \mathrm{~d} k\right| \Psi\right\rangle \leq|B|^{-1}\left\langle\Psi\left|\int \widehat{W}_{B}(k) a\left(Q\left(\chi_{B} e^{i k x}\right)\right)^{*} n a\left(Q\left(\chi_{B} e^{i k x}\right)\right) \mathrm{d} k\right| \Psi\right\rangle
$$




$$
\begin{aligned}
& \leq C|B|^{-1} a\left\langle\Psi\left|n n_{+}\right| \Psi\right\rangle \\
& \leq C \rho^{2} a|B| \sqrt{\rho a^{3}} \mathcal{S} \\
& \leq C \rho^{2} a|B| \frac{a}{R},
\end{aligned}
$$

where we also used Lemma 6.2 , that $n \leq C \rho|B|$ and Condition 5 .

We now show 122 . Repeating the estimate for the lower bound on $H_{\text {Quad }}$ with only half of the term in 122 included would again give a lower bound of order $\rho^{2}|B| a \frac{a}{R}$ because the second Born approximation to $a$ would be calculated wrong to this order. If $(122)$ would not hold this would give that $\left\langle\Psi, H_{\text {Quad }} \Psi\right\rangle \geq 0$, which contradicts the assumption in (117).

CONDITION 6: We require
(i) $\frac{a}{R} \mathcal{M}^{-2}<C \sqrt{\rho a^{3}}(\sqrt{\rho a} d s \ell)^{-3}$,
(ii) $\frac{a}{R} \mathcal{M}^{-2}<\delta \sqrt{\rho a^{3}}$,
(iii) $\rho|B| \sqrt{\rho a^{3}} \mathcal{S} \leq \mathcal{M}<\delta \rho|B|$.

We note that (ii) actually is a stronger requirement than (i).

For convenience we collect properties of the $n_{+}$-localized state $\widetilde{\psi}$ provided by Theorem 6.5 .

THEOREM 6.7 (Properties of a $n_{+}$-localized state $\widetilde{\psi}$ ).

If $\Psi$ is an n-particle state satisfying (117) and $\mathcal{M}$ satisfies Condition 6 , we obtain from Theorem 6.5 an n-particle state $\widetilde{\psi}$ which satisfies:

(i) Condition 4 is satisfied by $\widetilde{\psi}$.

(ii) $\left\langle\widetilde{\psi}, n_{+} \widetilde{\psi}\right\rangle \leq C \rho|B| \sqrt{\rho a^{3}} \mathcal{S} \quad$ and $\quad\langle\widetilde{\psi},|n-\rho| B|| \widetilde{\psi}\rangle \leq C \rho|B|\left(\rho a^{3}\right)^{\frac{1}{4}} \mathcal{S}^{\frac{1}{2}}$.

(iii) The requirement $n|B|^{-1} \leq c\left(a R^{2}\right)^{-1}$ in Lemma 4.6 holds.

(iv) The $n_{+}$eigenvalues of $\widetilde{\psi}$ are localized to an interval of length $\mathcal{M}$.

(v) $\left\langle\widetilde{\psi}, n_{+}^{2} \widetilde{\psi}\right\rangle \leq C \mathcal{M}\left\langle\widetilde{\psi}, n_{+} \widetilde{\psi}\right\rangle$.

(vi) $\left\langle\Psi,\left(-\varepsilon_{0} \Delta_{u}^{\mathcal{N}}+H_{B}\right) \Psi\right\rangle \geq\left\langle\widetilde{\psi},\left(-\varepsilon_{0} \Delta_{u}^{\mathcal{N}}+H_{B}\right) \widetilde{\psi}\right\rangle-C \delta \rho^{2} a \sqrt{\rho a^{3}}|B|$.

Here $\Delta_{u}^{\mathcal{N}}$ denotes the Neumann Laplacian described on page 18 and $\mathcal{S}$ is as defined in Lemma 6.2.

Proof. (i): This follows from equations (117), 118), Lemma 6.6 and Conditions 1 and 6(i). (ii): This follows from Lemma 6.2 and Lemma 6.4 , which by (i) apply to the state $\widetilde{\psi}$.

(iii): This follows from (ii) and the upper bound on $R$ in (5).

(iv): This property of $\widetilde{\psi}$ follows from Lemma 6.5 .

(v): By (ii) and Condition 6 (iii) the largest $n_{+}$-eigenvalue of $\widetilde{\psi}$ is bounded by $C \mathcal{M}$. This yields the stated estimate.

(vi): Apply Lemma 6.6, with $H_{B}$ replaced by $-\varepsilon_{0} \Delta_{u}^{\mathcal{N}}+H_{B}$, and Condition 6 (ii) to (118). 
The following lemma will be used to control the expectation of the second term in $\mathcal{Q}_{3}$, see 64, in the state $\widetilde{\psi}$ when $\varepsilon_{3} \gg 1$.

LEMMA 6.8. With

$$
h:=\sum_{i=1}-\varepsilon_{0} \Delta_{u, i}^{\mathcal{N}}-\varepsilon_{3} \sum_{i<j} Q_{j} Q_{i} w_{B}\left(x_{i}, x_{j}\right) Q_{i} Q_{j}
$$

we have

$$
\langle\psi, h \psi\rangle \geq 0
$$

provided all $n_{+}$-eigenvalues of $\psi$ satisfy $\varepsilon_{0} n_{+} \geq C_{0} \varepsilon_{3} \frac{a}{R} n_{+}^{2}$ with $C_{0}$ sufficiently large and depending only on $v_{1}$.

Proof. The operator $h$ acts in Fock space and commutes with $n_{+}$. In fact, $h$ only depends on the excited particles in the following sense. We can identify the Fock space as $\mathcal{F}_{B}\left(L^{2}(B)\right)=$ $\mathcal{F}_{B}\left(Q L^{2}(B)\right) \otimes \mathcal{F}_{B}\left(P L^{2}(B)\right)$. In this representation $h$ is an operator acting only on the first factor. In a fixed $n_{+}$subspace of this factor the operator has the form

$$
h=\sum_{i=1}^{n_{+}}\left(-\varepsilon_{0} \Delta_{i}^{\mathcal{N}}-\varepsilon_{3} \sum_{j=i+1}^{n_{+}} Q_{i} Q_{j} w_{B}\left(x_{i}, x_{j}\right) Q_{i} Q_{j}\right)
$$

If $\psi$ is in this subspace, we have

$$
\langle\psi, h \psi\rangle=\sum_{i=1}^{n_{+}}\left(\left\langle\psi,-\varepsilon_{0} \Delta_{i}^{\mathcal{N}} \psi\right\rangle-\varepsilon_{3} \sum_{j=i+1}^{n_{+}}\left\langle\psi, w_{B}\left(x_{i}, x_{j}\right) \psi\right\rangle\right) .
$$

Note that a function $\phi$, orthogonal to constants, i.e., $\phi \in Q L^{2}(B)$ satisfies the Sobolev inequality $\left\langle\phi,-\Delta^{\mathcal{N}} \phi\right\rangle \geq C\|\phi\|_{6}^{2}$. This implies that if $\psi$ is normalized, then

$$
\begin{aligned}
& \sum_{i=1}^{n_{+}} \int \varepsilon_{0}\left|\nabla_{i} \psi\left(x_{1}, \ldots, x_{n_{+}}\right)\right|^{2}-\varepsilon_{3} \sum_{j=i+1}^{n_{+}} \omega_{B}\left(x_{i}, x_{j}\right)\left|\psi\left(x_{1}, \ldots, x_{n_{+}}\right)\right|^{2} \mathrm{~d} x_{1} \cdots \mathrm{d} x_{n_{+}} \\
= & n_{+} \int \varepsilon_{0}\left|\nabla_{1} \psi\left(x_{1}, \ldots, x_{n_{+}}\right)\right|^{2}-\varepsilon_{3} \frac{\left(n_{+}-1\right)}{2} \omega_{B}\left(x_{1}, x_{2}\right)\left|\psi\left(x_{1}, \ldots, x_{n_{+}}\right)\right|^{2} \mathrm{~d} x_{1} \cdots \mathrm{d} x_{n_{+}} \\
\geq & \int\left(C \varepsilon_{0} n_{+}-\varepsilon_{3} \frac{n_{+}^{2}}{2}|| \omega_{B}\left(\cdot, x_{2}\right)||_{\frac{3}{2}}\right)\left(\int\left|\psi\left(x_{1}, \ldots, x_{n_{+}}\right)\right|^{6} \mathrm{~d} x_{1}\right)^{\frac{1}{3}} \mathrm{~d} x_{2} \cdots \mathrm{d} x_{n_{+}} \\
\geq & \left(C \varepsilon_{0} n_{+}-C \varepsilon_{3} n_{+}^{2} \frac{a}{R}\right) \int\left(\int\left|\psi\left(x_{1}, \ldots, x_{n_{+}}\right)\right|^{6} \mathrm{~d} x_{1}\right)^{\frac{1}{3}} \mathrm{~d} x_{2} \cdots \mathrm{d} x_{n_{+}} \\
\geq & 0,
\end{aligned}
$$


where we have used that $\int \omega_{B}(x, y)^{\frac{3}{2}} \mathrm{~d} x \leq C \int W(x-y)^{\frac{3}{2}} \mathrm{~d} x \leq C \int v_{R}(x)^{\frac{3}{2}} \mathrm{~d} x \leq C\left(\frac{a}{R}\right)^{\frac{3}{2}}$.

By Condition 6 (iii) Lemma 6.8 is applicable to $\widetilde{\psi}$ if we introduce the below condition.

CONDITION 7: We require

$$
\varepsilon_{0} \geq C \varepsilon_{3} \frac{a}{R} \mathcal{M}
$$

\section{Proof of Theorem 2.1}

Our goal has been to prove Theorem 2.1 as we have already concluded on page 7 that it implies the main result, Theorem 1.1. We now put the ingredients together to prove Theorem 2.1. We start with the lower bound on $H_{\rho}$ in Theorem 3.10 (here $H_{B}$ is $H_{u}$ ). We then focus on $n_{+}$-localized states, $\widetilde{\psi}$, provided by Theorem 6.7 and use the decomposition

$$
\left\langle\widetilde{\psi}\left|-\varepsilon_{0} \Delta_{u}^{\mathcal{N}}+H_{u}\right| \widetilde{\psi}\right\rangle=\left\langle\widetilde{\psi}\left|\left(H_{\mathrm{Quad}}+\mathcal{Q}_{1}^{\prime}\right)+\left(-\varepsilon_{0} \Delta_{u}^{\mathcal{N}}+H_{u}-H_{\mathrm{Quad}}-\mathcal{Q}_{1}^{\prime}\right)\right| \widetilde{\psi}\right\rangle
$$

We use Lemma 4.4 and Lemma 4.6 to show that the first parenthesis of the r.h.s. of 126 is consistent with (6) (up to a term that cancels with a corresponding positive term in the second parenthesis of (126)). Using Lemma 6.8 we show that the remaining terms in (126) are of higher order.

As a prelude to the proof of Theorem 2.1 we show that our parameters $d, s, \ell, \mathcal{M}, \varepsilon_{0}, \varepsilon_{T}, \varepsilon_{3}$ and the integer $M$ can be chosen such that all imposed conditions are satisfied. We choose our parameters in such a way that the error terms appearing in the proof of Theorem 2.1 are easily seen to be of size $o\left((\rho a)^{\frac{5}{2}}\right)$. However, our choice is certainly not optimal in that it does not give an optimal bound on our error terms. In particular, we decided not to determine an optimal value for $N$ below. Our choice for $\mathcal{M}$ in Lemma 7.1 and why we have $\eta<\frac{1}{30}$ in Assumption 2 will be discussed on page 52 .

LEMMA 7.1. For any $0<\delta<\delta_{\eta}<1$ Conditions 1, 2, 3, 5, 6, and 7 are satisfied if we take $N$ to be sufficiently large, $\rho$ sufficiently small, denote $X:=\max \left\{\left(\rho a^{3}\right)^{-\frac{3}{10}} \frac{a}{R}, R \sqrt{\rho a}\right\}$ and choose

$$
\begin{gathered}
d=X^{\frac{4}{N}}, \quad s=X^{\frac{3}{2 N}}, \quad \ell=(\rho a)^{-\frac{1}{2}} X^{-\frac{2}{N}}, \quad \delta \varepsilon_{T}=(\sqrt{\rho a} d \ell)^{2}=X^{\frac{4}{N}}, \\
\varepsilon_{0}=C \varepsilon_{3} \frac{a}{R} \mathcal{M}, \quad \varepsilon_{3}=\mathcal{S} X^{-\frac{1}{N}}=X^{-\frac{31}{2 N}}, \quad \mathcal{M}=\left(\frac{R}{a}\right)^{\frac{1}{3}}, \quad M=20 .
\end{gathered}
$$

Here $M$ was introduced in (13) and $\mathcal{S}=\rho a \ell^{2}(\sqrt{\rho a} d s \ell)^{-3}=X^{-\frac{29}{2 N}}$ was defined in Lemma 6.2. 
Proof. Note that $\lim _{\rho \rightarrow 0} X=0$ by Assumption 2. Since $4>\frac{3}{2}$ we have $d<\delta s$, as required in Condition 1, provided $\rho$ is sufficiently small.

The requirement $\delta<\delta_{\eta}$ is only relevant for the second item in Condition 3, which we check now. We may assume that $\frac{R}{a} \leq\left(\rho a^{3}\right)^{-\frac{1}{5}}$ such that $X=\left(\rho a^{3}\right)^{-\frac{3}{10}} \frac{a}{R}$. By Assumption 2 we have $\left(\rho a^{3}\right)^{-\frac{1}{5}} \leq X^{-\alpha_{\eta}}$ for some $\alpha_{\eta}>0$. Thus

$$
\begin{aligned}
(\sqrt{\rho a} d \ell)^{2} \ln \left(\frac{d s \ell}{R}\right)(d s \ell \sqrt{\rho a})^{-1} & =\sqrt{\rho a} d s^{-1} \ell \ln \left(\frac{d s \ell}{R}\right) \\
& =X^{\frac{-2+4-\frac{3}{2}}{N}} \ln \left(X^{1+\frac{7}{2 N}}\left(\rho a^{3}\right)^{\frac{3}{10}-\frac{1}{2}}\right) \\
& \leq X^{\frac{1}{2 N}} \ln \left(X^{1+\frac{7}{2 N}-\alpha_{\eta}}\right) .
\end{aligned}
$$

The other conditions are checked in a similar but simpler manner.

Proof of Theorem 2.1. We choose our parameters as prescribed in Lemma 7.1. From Theorem 3.10 we obtain with $\Lambda^{\prime}:=\Lambda+\left[-\frac{\ell}{2}, \frac{\ell}{2}\right]^{3}$ the following lower bound on the background Hamiltonian, $H_{\rho}$, which was defined via (9), in terms of the localized Hamiltonian, $H_{u}$, which was defined in (50),

$$
H_{\rho} \geq \int_{\ell^{-1} \Lambda^{\prime}}\left(-\varepsilon_{0} \Delta_{u}^{\mathcal{N}}+H_{u}\right) \mathrm{d} u
$$

This inequality is to be understood in the sense of quadratic forms and since all operators commute with the particle number, $n$, it suffices to consider states with fixed particle number. Since we in Theorem 2.1 are interested in the ground state energy density after passing to the thermodynamic limit it remains to show that

$$
\left\langle\Psi,\left(-\varepsilon_{0} \Delta_{u}^{\mathcal{N}}+H_{u}\right) \Psi\right\rangle \ell^{-3} \geq 4 \pi \rho^{2}\left(a_{2}+\frac{128}{15 \sqrt{\pi}} a\left(\sqrt{\rho a^{3}}+o\left(\sqrt{\rho a^{3}}\right)\right)\right),
$$

for any state $\Psi$ on the box $B(u)$ with fixed particle number $n$. Note that in case $\Psi$ does not satisfy 130, then $\Psi$ satisfies 117 .

Hence, provided $\mathcal{M}$ satisfied Condition 6, we obtain from Theorem 6.7 a $n_{+}$-localized state $\widetilde{\psi}$ which, in particular, satisfies

$$
\left\langle\widetilde{\psi}, n_{+}^{2} \widetilde{\psi}\right\rangle \leq C \mathcal{M}\left\langle\widetilde{\psi}, n_{+} \widetilde{\psi}\right\rangle
$$

and

$$
\left\langle\Psi,\left(-\varepsilon_{0} \Delta_{u}^{\mathcal{N}}+H_{u}\right) \Psi\right\rangle \geq\left\langle\widetilde{\psi},\left(-\varepsilon_{0} \Delta_{u}^{\mathcal{N}}+H_{u}\right) \widetilde{\psi}\right\rangle+o\left(\rho^{2} a \sqrt{\rho a^{3}}|B|\right) .
$$


Thus it suffices to show that

$$
\left\langle\widetilde{\psi},\left(-\varepsilon_{0} \Delta_{u}^{\mathcal{N}}+H_{u}\right) \widetilde{\psi}\right\rangle \ell^{-3} \geq 4 \pi \rho^{2}\left(a_{2}+\frac{128}{15 \sqrt{\pi}} a\left(\sqrt{\rho a^{3}}+o\left(\sqrt{\rho a^{3}}\right)\right)\right) .
$$

With the notation from Section 4 we decompose $H_{u}$ (see (66) ) as

$$
H_{u}=\left(\sum_{i=1}^{N} \mathcal{T}_{u, i}+\mathcal{Q}_{1}^{\prime}+\mathcal{Q}_{2}^{\prime}\right)+\left(\mathcal{Q}_{0}+\mathcal{Q}_{1}^{\prime \prime}+\mathcal{Q}_{2}^{\prime \prime}+\mathcal{Q}_{3}+\mathcal{Q}_{4}\right)
$$

Using this decomposition the estimate in 133 is a consequence of Lemma 7.2, Lemma 7.3 and Lemma 7.4 below.

LEMMA 7.2. Let $\widetilde{\psi}$ be some n-particle state on $B(u)$ with properties as in Theorem 6.7 let $h_{0}$ be defined as in (83) and choose parameters as in Lemma 7.1. Then

$$
\left\langle\widetilde{\psi},\left(\sum_{i=1}^{N} \mathcal{T}_{u, i}+\mathcal{Q}_{1}^{\prime}+\mathcal{Q}_{2}^{\prime}\right) \widetilde{\psi}\right\rangle \ell^{-3} \geq \frac{1}{2}(2 \pi)^{-3} \int_{\mathbb{R}^{3}} h_{0}(k) \mathrm{d} k-\left|n-\rho \ell^{3}\right|^{2} \frac{a}{\ell^{6}}+o\left((\rho a)^{\frac{5}{2}}\right)
$$

LEMMA 7.3. Let $\widetilde{\psi}$ be some $n$-particle state on $B(u)$ with properties as in Theorem 6.7. let $h_{0}$ be defined as in (83) and choose parameters as in Lemma 7.1. Then

$$
\left\langle\widetilde{\psi}, \frac{1}{2}(2 \pi)^{-3} \int_{\mathbb{R}^{3}} h_{0}(k) \mathrm{d} k \widetilde{\psi}\right\rangle \ell^{-3} \geq 4 \pi \rho^{2}\left(a_{2}+\frac{128}{15 \sqrt{\pi}} a\left(\sqrt{\rho a^{3}}+o\left(\sqrt{\rho a^{3}}\right)\right)\right) .
$$

LEMMA 7.4. Let $\widetilde{\psi}$ be some n-particle state on $B(u)$ with properties as in Theorem 6.7 and choose parameters as in Lemma 7.1. Then

$$
\left\langle\widetilde{\psi},\left(-\varepsilon_{0} \Delta_{u}^{\mathcal{N}}+\mathcal{Q}_{0}+\mathcal{Q}_{1}^{\prime \prime}+\mathcal{Q}_{2}^{\prime \prime}+\mathcal{Q}_{3}+\mathcal{Q}_{4}\right) \widetilde{\psi}\right\rangle \ell^{-3} \geq\left|n-\rho \ell^{3}\right|^{2} \frac{a}{\ell^{6}}+o\left((\rho a)^{\frac{5}{2}}\right) .
$$

Proof of Lemma 7.2. Recall that $\mathcal{T}_{B}$ is defined via (53) and 41) as $\left(1-\varepsilon_{0}\right) \widehat{\mathcal{T}}$. We note that the second term in $\widehat{\mathcal{T}}$ is positive and that $\left(1-\varepsilon_{0}\right)$ times the last term is part of what we in (71) denoted as the quadratic Hamiltonian, $H_{\text {Quad }}$, using the notation in 68). Thus we have

$$
\left\langle\widetilde{\psi},\left(\sum_{i=1}^{N} \mathcal{T}_{B, i}+\mathcal{Q}_{1}^{\prime}+\mathcal{Q}_{2}^{\prime}\right) \widetilde{\psi}\right\rangle \ell^{-3} \geq\left\langle\widetilde{\psi},\left(H_{\mathrm{Quad}}+\mathcal{Q}_{1}^{\prime}\right) \widetilde{\psi}\right\rangle \ell^{-3}+C \ell^{-5} n_{+}
$$

We proceed and apply Lemma 4.4 with $\sigma=1$ to obtain

$$
\left\langle\widetilde{\psi},\left(H_{\mathrm{Quad}}+\mathcal{Q}_{1}^{\prime}\right) \widetilde{\psi}\right\rangle \ell^{-3} \geq \frac{1}{2}(2 \pi)^{-3} \int_{\mathbb{R}^{3}} h_{1}(k) \mathrm{d} k \ell^{-3}-C n_{+} \frac{a}{|B|^{2}},
$$


with $h_{1}$ as defined in 83 . By Condition 1 we have $C \ell^{-5} n_{+}-C n_{+} \frac{a}{|B|^{2}} \geq 0$. We use Lemma 4.6 . which is applicable by Theorem 6.7 (iii), to estimate the integral over $h_{1}$ as explained on page 32 and get

$$
\frac{1}{2}(2 \pi)^{-3} \int_{\mathbb{R}^{3}} h_{1}(k) \mathrm{d} k \ell^{-3} \geq-|n-\rho| B||^{2} \frac{a}{|B|^{2}}+\frac{1}{2}(2 \pi)^{-3} \int_{\mathbb{R}^{3}} h_{0}(k) \mathrm{d} k \ell^{-3}+o\left((\rho a)^{\frac{5}{2}}\right) .
$$

Proof of Lemma 7.3. The value $4 \pi \frac{128}{15 \sqrt{\pi}}$ for the LHY-term is obtained by integrating over values of $k$ close to $\sqrt{\rho a}$ after subtracting a part related to the (negative) second Born term. This term will again be added in (142) and the complete Born term will be obtained in (144). In other words, we prove Lemma 7.3 by showing the two estimates

$$
\begin{aligned}
& \frac{1}{2}(2 \pi)^{-3} \int_{(s \ell)^{-1}<|k|<(d \ell)^{-1}} h_{0}(k)+\frac{|k|^{2}}{2} \frac{n^{2} \widehat{W}(k)^{2}}{|B| \tau_{B}\left(k^{2}\right)^{2}} \mathrm{~d} k|B|^{-1} \\
& \geq 4 \pi \rho^{2} \frac{128}{15 \sqrt{\pi}} a \sqrt{\rho a^{3}}+o\left((\rho a)^{\frac{5}{2}}\right)
\end{aligned}
$$

and

$$
\begin{aligned}
\frac{1}{2}(2 \pi)^{-3} & \left.\underset{(s \ell)^{-1}<|k|<(d \ell)^{-1}}{-}-\int_{|k|^{2}} \frac{n^{2} \widehat{W}(k)^{2}}{|B| \tau_{B}\left(k^{2}\right)^{2}} \mathrm{~d} k+\int_{|k|<(s \ell)^{-1}} h_{0} \mathrm{~d} k+\int_{|k|>(d \ell)^{-1}} h_{0} \mathrm{~d} k\right)|B|^{-1} \\
& \geq-\frac{1}{4}(2 \pi)^{-3} \int_{|k|>(s \ell)^{-1}}|k|^{2} \frac{n^{2} \widehat{W}(k)^{2}}{|B|^{2} \tau_{B}\left(k^{2}\right)^{2}}+o\left((\rho a)^{\frac{5}{2}}\right) \\
& \geq-\frac{1}{4}(2 \pi)^{-3} \rho^{2} \frac{1}{R} \int \frac{\widehat{v}_{1}(k)^{2}}{|k|^{2}} \mathrm{~d} k+o\left((\rho a)^{\frac{5}{2}}\right)
\end{aligned}
$$

Proof of (141). By Theorem 6.7 (iii) we may lower bound the integral over $h_{0}$ using Lemma 4.6. We get

$$
\begin{aligned}
& \frac{1}{2}(2 \pi)^{-3} \int_{(s \ell)^{-1}<|k|<(d \ell)^{-1}} h_{0}(k)+\frac{|k|^{2}}{2} \frac{n^{2} \widehat{W}(k)^{2}}{|B| \tau_{B}\left(k^{2}\right)^{2}} \mathrm{~d} k|B|^{-1} \\
\geq & \frac{1}{2}(2 \pi)^{-3} \int_{(s \ell)^{-1}<|k|<(d \ell)^{-1}}|k|^{2}\left(\sqrt{1+2 \frac{n \widehat{W}(k)}{|B| \tau_{B}\left(k^{2}\right)}}-1-\frac{n \widehat{W}(k)}{|B| \tau_{B}\left(k^{2}\right)}+\frac{1}{2} \frac{n^{2} \widehat{W}(k)^{2}}{|B|^{2} \tau_{B}\left(k^{2}\right)^{2}}\right) \mathrm{d} k \\
\geq & \frac{1}{2}(2 \pi)^{-3} \int_{(s \ell)^{-1}<|k|<(d \ell)^{-1}}|k|^{2}\left(\sqrt{1+2 \frac{n \widehat{W}(k)}{|B||k|^{2}}}-1-\frac{n \widehat{W}(k)}{|B||k|^{2}}+\frac{1}{2} \frac{n^{2} \widehat{W}(k)^{2}}{|B|^{2}|k|^{4}}\right) \mathrm{d} k
\end{aligned}
$$




$$
\begin{aligned}
\geq & \frac{1}{2}(2 \pi)^{-3} \int_{(s \ell)^{-1}<|k|<(d \ell)^{-1}}|k|^{2}\left(\sqrt{1+2 \frac{n}{|B|} \widehat{v_{R}}(0)|k|^{-2}}-1-\frac{n}{|B|} \widehat{v_{R}}(0)|k|^{-2}+\frac{1}{2} \frac{n^{2}}{|B|^{2}} \widehat{v_{R}}(0)^{2}|k|^{-4}\right) \mathrm{d} k \\
& -C \rho^{2} a \sqrt{\rho a^{3}} X^{2+\frac{2}{N}}
\end{aligned}
$$

In the first inequality we used (A.4) to conclude that the lower bound on $h_{0}(k)$ in A.1) is negative and then replace $\tau_{B}\left(k^{2}\right)$ by the larger value $|k|^{2}$.

Then we used that if $f(x)=\sqrt{1+2 x}-1-x+\frac{1}{2} x^{2}$, then $f^{\prime}(x)=(1+2 x)^{-\frac{1}{2}}-1+x$ and $0 \leq \frac{1}{1+x}-1+x \leq f^{\prime}(x) \leq \min \left\{x, \frac{3}{2} x^{2}\right\}$ for $x \geq 0$. To obtain the error term in 146) we recall that if $|k|<R^{-1}$, then $\widehat{v_{R}}(0)-\widehat{W}(k) \leq \frac{1}{2}(k R)^{2} \int v_{R}(x) \mathrm{d} x$ by 19 ) and (86). Then we estimate

$$
\begin{aligned}
& \int_{(s \ell)^{-1}<|k|<(\rho a)^{\frac{1}{2}}}|k|^{2} \frac{\rho a}{|k|^{2}} \rho a R^{2} \mathrm{~d} k+\int_{(\rho a)^{\frac{1}{2}}<|k|<(d \ell)^{-1}}|k|^{2}\left(\frac{\rho a}{|k|^{2}}\right)^{2} \rho a R^{2} \mathrm{~d} k \\
& \leq C(\rho a)^{\frac{7}{2}} R^{2}+C(\rho a)^{3} R^{2}(d \ell)^{-1} \leq C(\rho a)^{3} R^{2}(d \ell)^{-1} \leq C \rho^{2} a \sqrt{\rho a^{3}} X^{2+\frac{2}{N}} .
\end{aligned}
$$

We recall that $\left|\frac{n}{|B|}-\rho\right| \leq C \rho\left(\rho a^{3}\right)^{\frac{1}{4}} \mathcal{S}^{\frac{1}{2}}$ by Theorem 6.7 (ii) and lower bound the integral appearing in 146 by

$$
\begin{aligned}
& \frac{1}{2}(2 \pi)^{-3} \int_{(s \ell)^{-1}<|k|<(d \ell)^{-1}}|k|^{2}\left(\sqrt{1+2 \rho \widehat{v_{R}}(0)|k|^{-2}}-1-\rho \widehat{v_{R}}(0)|k|^{-2}+\frac{1}{2} \rho^{2} \widehat{v_{R}}(0)^{2}|k|^{-4}\right) \mathrm{d} k \\
& \quad-C(\rho a)^{\frac{5}{2}}\left(\rho a^{3}\right)^{\frac{1}{4}} \mathcal{S}^{\frac{1}{2}} \\
& =\frac{1}{2}(2 \pi)^{-3}\left(\rho \widehat{v_{R}}(0)\right)^{\frac{5}{2}} \int-|k|^{2}-1+|k|^{2} \sqrt{1+2|k|^{-2}}+\frac{1}{2}|k|^{-2} \mathrm{~d} k-C(\rho a)^{\frac{5}{2}}\left(\rho a^{3}\right)^{\frac{1}{4}} \mathcal{S}^{\frac{1}{2}} \\
& \quad(s \ell)^{-1}\left(\rho \widehat{v_{R}}(0)\right)^{-\frac{1}{2}}<|k|<(d \ell)^{-1}\left(\rho \widehat{v_{R}}(0)\right)^{-\frac{1}{2}} \\
& \geq 4 \pi \rho^{2} a \frac{128}{15 \sqrt{\pi}} \sqrt{\rho a^{3}}-C(\rho a)^{\frac{5}{2}}\left[(s \ell)^{-1}(\rho a)^{-\frac{1}{2}}+d \ell(\rho a)^{\frac{1}{2}}+\left(\rho a^{3}\right)^{\frac{1}{4}} \mathcal{S}^{\frac{1}{2}}\right] \\
& =4 \pi \rho^{2} a \frac{128}{15 \sqrt{\pi}} \sqrt{\rho a^{3}}-C(\rho a)^{\frac{5}{2}}\left[X^{\frac{1}{2 N}}+X^{\frac{2}{N}}+\left(\rho a^{3}\right)^{\frac{1}{4}} X^{-\frac{29}{4 N}}\right] .
\end{aligned}
$$

Here we used the identity $\int_{\mathbb{R}^{3}}-|k|^{2}-1+|k|^{2} \sqrt{1+2|k|^{-2}}+\frac{1}{2}|k|^{-2} \mathrm{~d} k=\frac{32}{15} \pi \sqrt{2}$, for which we note that the integrand is bounded by $\frac{1}{2}|k|^{-2}$ for small $|k|$ and $C|k|^{-4}$ for large $|k|$.

Proof of 144. First we show how to arrive at (143). We use (A.3) for $(d \ell)^{-1}<|k|<R^{-1}$, where $\widehat{W}(k) \geq 0$, and A.4 for $|k| \geq R^{-1}$ to obtain the estimate

$$
\frac{1}{2}(2 \pi)^{-3} \int_{|k|>(d \ell)^{-1}} h_{0}(k) \mathrm{d} k|B|^{-1} \geq-\frac{1}{4}(2 \pi)^{-3} \int_{|k|>(d \ell)^{-1}}|k|^{2} \frac{n^{2} \widehat{W^{2}}(k)}{|B|^{2} \tau_{B}\left(k^{2}\right)^{2}} \mathrm{~d} k-C \int_{|k|>R^{-1}} \frac{n^{3}|\widehat{W}(k)|^{3}}{|B|^{3}|k|^{4}} \mathrm{~d} k
$$


By A.19 and Theorem 6.7 (ii) the second term in 148 is bounded by $C(\rho a)^{3} R$.

For $|k|<(s \ell)^{-1}$ Theorem 6.7 (ii) and A.17) yield

$$
\int_{|k|<(s \ell)^{-1}} h_{0}(k) \mathrm{d} k|B|^{-1} \geq-C \frac{n}{|B|} a(s \ell)^{-3} \geq-C \rho a(s \ell)^{-3}=-C \rho^{2} a \sqrt{\rho a^{3}} X^{\frac{3}{2 N}}
$$

To arrive at (144) from (143) we use (A.22), A.23) and Theorem 6.7 (ii) and obtain

$$
\begin{aligned}
& -\frac{1}{4}(2 \pi)^{-3} \int_{|k|>(s \ell)^{-1}}|k|^{2} \frac{n^{2} \widehat{W}^{2}(k)}{|B|^{2} \tau_{B}\left(k^{2}\right)^{2}} \mathrm{~d} k \\
\geq & -\frac{1}{4}(2 \pi)^{-3} \rho^{2} \int \frac{\widehat{v_{R}}(k)^{2}}{|k|^{2}} \mathrm{~d} k-C \rho^{2} a \varepsilon_{0} \frac{a}{R}-C \rho^{2} a \frac{a}{s \ell} \ln \left(\frac{s \ell}{R}\right) \\
& -C \rho^{2} a \varepsilon_{T} \frac{a}{d s \ell} \ln \left(\frac{d s \ell}{R}\right)-C \rho^{2} a\left(\rho a^{3}\right)^{\frac{1}{4}} \mathcal{S}^{\frac{1}{2}} \frac{a}{R} \\
\geq & -\frac{1}{4}(2 \pi)^{-3} \rho^{2} \int \frac{\widehat{v_{R}}(k)^{2}}{|k|^{2}} \mathrm{~d} k-C \rho^{2} a \sqrt{\rho a^{3}}\left[X^{\frac{5}{3}-\frac{31}{2 N}}+X^{\frac{1}{2 N}} \ln \left(X^{1-\frac{1}{2 N}}\left(\rho a^{3}\right)^{-\frac{1}{5}}\right)\right] \\
& -C \rho^{2} a \sqrt{\rho a^{3}}\left[X^{\frac{1}{2 N}} \ln \left(X^{1+\frac{7}{2 N}}\left(\rho a^{3}\right)^{-\frac{1}{5}}\right)+\left(\rho a^{3}\right)^{\frac{1}{20}} X^{-\frac{29}{4 N}}\right] .
\end{aligned}
$$

The error terms in 150 are small since we required $\eta<\frac{1}{30}$ in Assumption 2 .

Proof of Lemma 7.4. Recall that Theorem 6.7 (ii) provides the bounds

$$
\left\langle\widetilde{\psi}, n_{+} \widetilde{\psi}\right\rangle \leq C \rho|B| \sqrt{\rho a^{3}} \mathcal{S} \quad \text { and } \quad\langle\widetilde{\psi},|n-\rho| B|| \widetilde{\psi}\rangle \leq C \rho|B|\left(\rho a^{3}\right)^{\frac{1}{4}} \mathcal{S}^{\frac{1}{2}}
$$

With $\varepsilon_{1}^{\prime \prime}=(\rho|B|)^{-\frac{1}{2}} \mathcal{M}^{\frac{1}{2}}$ and Condition 6 (iii) we obtain from (57), Lemma 4.2 and 63 )

$$
\begin{aligned}
& \left\langle\widetilde{\psi}\left|\mathcal{Q}_{0}+\mathcal{Q}_{1}^{\prime \prime}+\mathcal{Q}_{2}^{\prime \prime}\right| \widetilde{\psi}\right\rangle|B|^{-1} \\
& \geq\left\langle\widetilde{\psi}\left|\left[|n-\rho| B||^{2}-C|n-\rho| B|| n_{+}+n_{+}^{2}-n_{0}-\varepsilon_{1}^{\prime \prime}\left(n_{+}+1\right) n_{0}-C \varepsilon_{1}^{\prime \prime} n_{+}^{2}-C n_{+}^{2}\right] \mathcal{U}_{B}\right| \widetilde{\psi}\right\rangle|B|^{-1} \\
& \geq\left.|n-\rho| B\right|^{2} \frac{a}{|B|^{2}}-C \rho^{2} a \sqrt{\rho a^{3}}\left(\rho a^{3}\right)^{\frac{1}{4}} \mathcal{S}^{\frac{3}{2}}-C \rho^{2} a \sqrt{\rho a^{3}} \mathcal{S}(\rho|B|)^{-\frac{1}{2}} \mathcal{M}^{\frac{1}{2}}-C \rho \frac{a}{|B|} \\
& \geq\left.|n-\rho| B\right|^{2} \frac{a}{|B|^{2}}-C \rho^{2} a \sqrt{\rho a^{3}} \mathcal{S}(\rho|B|)^{-\frac{1}{2}} \mathcal{M}^{\frac{1}{2}}-C \rho \frac{a}{|B|} \\
& \geq\left.|n-\rho| B\right|^{2} \frac{a}{|B|^{2}}-C \rho^{2} a \sqrt{\rho a^{3}}\left[X^{-\frac{23}{2 N}}\left(\rho a^{3}\right)^{\frac{1}{6}}+X^{\frac{6}{N}}\right]
\end{aligned}
$$

By Theorem 6.7 we have $\left\langle\widetilde{\psi}, n_{+}^{2} \widetilde{\psi}\right\rangle \leq C \mathcal{M}\left\langle\widetilde{\psi}, n_{+} \widetilde{\psi}\right\rangle$. Thus Condition 7 ensures that Lemma 6.8 may be applied to the state $\widetilde{\psi}$ and we obtain

$$
\left\langle\widetilde{\psi},\left(\mathcal{Q}_{3}-\varepsilon_{0} \Delta_{u}^{\mathcal{N}}\right) \widetilde{\psi}\right\rangle|B|^{-1} \geq\left\langle\widetilde{\psi},\left(-C \varepsilon_{3}^{-1} n n_{+} \mathcal{U}_{B}-\varepsilon_{0} \Delta_{u}^{\mathcal{N}}-\varepsilon_{3} Q Q w_{B}(x, y) Q Q\right) \widetilde{\psi}\right\rangle|B|^{-1}
$$




$$
\geq-C \rho^{2} a\left(\rho a^{3}\right)^{\frac{1}{2}} \mathcal{S} \varepsilon_{3}^{-1}=-C \rho^{2} a\left(\rho a^{3}\right)^{\frac{1}{2}} X^{\frac{1}{N}}
$$

Finally we note that $\left\langle\widetilde{\psi}, \mathcal{Q}_{4} \widetilde{\psi}\right\rangle$ is defined to be positive in 65 .

Now we discuss why we have $\eta=\frac{1}{30}$ in Assumption 2 and why we chose $\mathcal{M}=\left(\frac{R}{a}\right)^{\frac{1}{3}}$ in Lemma 7.1. Combining (118) and Lemma 6.6, respectively (149) and (128), we obtain the error terms

$$
C \rho^{2} a \frac{a}{R} \mathcal{M}^{-2} \quad \text { and } \quad C \rho^{2} a \varepsilon_{0} \frac{a}{R} \geq C \rho^{2} a\left(\frac{a}{R}\right)^{2} \mathcal{M}
$$

Optimizing $C \rho^{2} a \frac{a}{R} \mathcal{M}^{-2}+C \rho^{2} a\left(\frac{a}{R}\right)^{2} \mathcal{M}$ gives $\mathcal{M}=\left(\frac{R}{a}\right)^{\frac{1}{3}}$ and the estimate

$$
\left(C\left(\rho a^{3}\right)^{-\frac{1}{2}} \frac{a}{R} \mathcal{M}^{-2}+C\left(\rho a^{3}\right)^{-\frac{1}{2}}\left(\frac{a}{R}\right)^{2} \mathcal{M}\right) \geq C\left[\frac{a}{R}\left(\rho a^{3}\right)^{-\frac{3}{10}}\right]^{\frac{5}{3}} .
$$

\section{A Appendix}

Throughout this appendix we will assume that $n \geq 1$ and that the condition on $n|B|^{-1}$ in Lemma 4.6 is satisfied, so that by Lemma 4.4 we have the lower bounds

$$
H_{\text {Quad }} \geq \frac{1}{2}(2 \pi)^{-3} \int_{\mathbb{R}^{3}} h_{0}(k) \mathrm{d} k-C n_{+} a \min \left\{R^{-3},|B|^{-1}\right\} \max \chi_{B}^{2}
$$

and

$$
h_{0}(k) \geq-\left(n^{-1} \tau_{B}\left(k^{2}\right)+|B|^{-1} \widehat{W}(k)-\sqrt{n^{-2} \tau_{B}\left(k^{2}\right)^{2}+2 n^{-1}|B|^{-1} \tau_{B}\left(k^{2}\right) \widehat{W}(k)}\right) n_{0} \int \chi_{B}^{2} .
$$

\section{A.1 Bounds for the Quadratic Part of $H_{B}$}

We estimate the operator valued integral $\frac{1}{2}(2 \pi)^{-3} \int h_{0}(k) \mathrm{d} k$ using the following facts. Around $x=0$ we can write $\sqrt{1+x}=1+\frac{1}{2} x-\frac{1}{8} x^{2}+\frac{1}{16} x^{3}+O\left(x^{4}\right)$ yielding the bounds

$$
\begin{aligned}
1+\frac{1}{2} x-C x^{2} \leq \sqrt{1+x} & \leq 1+\frac{1}{2} x-\frac{1}{8} x^{2}+C|x|^{3} \\
\sqrt{1+x} & \left.\geq 1+\frac{1}{2} x-\frac{1}{8} x^{2} \quad \text { (if and only if } x \geq 0\right) \\
1+\frac{1}{2} x-\frac{1}{8} x^{2}-C|x|^{3} \leq \sqrt{1+x} & \leq 1+\frac{1}{2} x .
\end{aligned}
$$


If $B$ is either a small or a large box, then, for all $k \in \mathbb{R}^{3}$, the parentheses in A.1 is positive. This is easy to see. If $B$ is a small box and $|k| \leq(d s \ell)^{-1}$, then $\tau_{B}\left(k^{2}\right)=0$ and $\widehat{W}(k)>0$ by (86) since $(d s \ell)^{-1}<R^{-1}$. For $|k|>(d s \ell)^{-1}$ we have $\tau_{B}\left(k^{2}\right)>0$ and apply (A.4). For the large box the claim is proven analogously. We may therefore replace $n_{0}$ by $n$ in (A.1) when bounding $h_{0}$ from below such that

$$
h_{0}(k) \geq-\left(n^{-1} \tau_{B}\left(k^{2}\right)+|B|^{-1} \widehat{W}(k)-\sqrt{n^{-2} \tau_{B}\left(k^{2}\right)^{2}+2 n^{-1}|B|^{-1} \tau_{B}\left(k^{2}\right) \widehat{W}(k)}\right) n \int \chi_{B}^{2} .
$$

Provided $\tau_{B}\left(k^{2}\right)>0$ we write

$$
h_{0}(k) \geq-\left(\tau_{B}\left(k^{2}\right)+n|B|^{-1} \widehat{W}(k)-\tau_{B}\left(k^{2}\right) \sqrt{1+\frac{2 n \widehat{W}(k)}{|B| \tau_{B}\left(k^{2}\right)}}\right) \int \chi_{B}^{2} .
$$

\section{A.1.1 Estimates on the Small Box}

$\tau_{B}\left(k^{2}\right)=0$ if $|k|<(d s \ell)^{-1}$ while $\widehat{W}(k)>0$ if $|k|<R^{-1}$. Since $\sqrt{1+x} \geq 1$ if $x \geq 0$, we have

$$
\int_{|k|<2(d s \ell)^{-1}} h_{0}(k) \mathrm{d} k \geq-\int_{|k|<2(d s \ell)^{-1}} \frac{n_{0}}{|B|} \widehat{W}(k) \mathrm{d} k \int \chi_{B}^{2} \geq-C \frac{n}{|B|} a(d s \ell)^{-3} \int \chi_{B}^{2}
$$

Using (A.3) for $2(d s \ell)^{-1}<|k|<R^{-1}$ and $(\mathrm{A} .4)$ for $|k|>R^{-1}$ gives

$$
\frac{1}{2}(2 \pi)^{-3} \int_{|k|>2(d s \ell)^{-1}} h_{0}(k) \mathrm{d} k \geq \frac{1}{2}(2 \pi)^{-3} \int_{|k|>2(d s \ell)^{-1}}-\frac{1}{2} \frac{n^{2}}{|B|^{2}} \frac{\widehat{W}(k)^{2}}{\tau_{B}\left(k^{2}\right)} \mathrm{d} k \int \chi_{B}^{2}-C \int_{|k|>R^{-1}} \frac{n^{3}}{|B|^{3}} \frac{|\widehat{W}(k)|^{3}}{\tau_{B}\left(k^{2}\right)^{2}} \mathrm{~d} k \int \chi_{B}^{2}
$$

Since $\tau_{B}=\left(1-\varepsilon_{0}\right)\left[|k|-(d s \ell)^{-1}\right]_{+}^{2} \geq C|k|^{2}$ if $|k| \geq 2(d s \ell)^{-1}$ and $|\widehat{W}(k)| \leq \widehat{W}(0) \leq C a$, it is easy to estimate the last term in A.8

$$
\int_{|k|>R^{-1}} \frac{n^{3}}{|B|^{3}} \frac{|\widehat{W}(k)|^{3}}{\tau_{B}\left(k^{2}\right)^{2}} \mathrm{~d} k \int \chi_{B}^{2} \leq C \int_{|k|>R^{-1}} \frac{n^{3}}{|B|^{3}} \frac{a^{3}}{|k|^{4}} \mathrm{~d} k \int \chi_{B}^{2} \leq C \frac{n^{3}}{|B|^{3}} a^{3} R \int \chi_{B}^{2}
$$

The integral $\int_{|k|>2(d s \ell)^{-1}} \frac{\widehat{W}(k)^{2}}{\tau_{B}\left(k^{2}\right)} \mathrm{d} k$ is related to the second Born term and we have to estimate it carefully. Recall that on the small box we $v_{R}(x) \leq W(x) \leq v_{R}(x)\left(1+C\left(\frac{R}{d \ell}\right)^{2}\right)$ by 20 . 
Thus we have

$$
\left\|v_{R}-W\right\|_{\frac{6}{5}} \leq C\left(\frac{R}{d \ell}\right)^{2}\left\|v_{R}\right\|_{\frac{6}{5}} \quad \text { and } \quad\left\|v_{R}\right\|_{\frac{6}{5}}=R^{-\frac{1}{2}}\left\|v_{1}\right\|_{\frac{6}{5}} .
$$

Since $v_{R}(x)=\frac{1}{R^{3}} v_{1}\left(\frac{x}{R}\right)$, we have $\widehat{v_{R}}(k)=\widehat{v_{1}}(R k)$ and that

$$
\int_{|k| \geq 2(d s \ell)^{-1}} \frac{\widehat{v_{R}}(k)^{2}}{|k|^{2}} \mathrm{~d} k=\frac{1}{R} \int_{|k| \geq 2(d s \ell)^{-1} R} \frac{\widehat{v}_{1}(k)^{2}}{|k|^{2}} \mathrm{~d} k
$$

For $f \in L^{\frac{6}{5}}\left(\mathbb{R}^{3}\right)$ a real space representation (see [21] Cor 5.10) followed by an application of the Hardy-Littlewood-Sobolev inequality gives

$$
\int|\widehat{f}(k)|^{2}|k|^{-2} \mathrm{~d} k \leq C\|f\|_{\frac{6}{5}}^{2}
$$

On the small box we have for $|k|>2(d s \ell)^{-1}$

$$
\tau_{B}\left(k^{2}\right)^{-1} \leq\left(1+C \varepsilon_{0}\right)|k|^{-2}+C(d s \ell)^{-1}|k|^{-3} .
$$

We use the Cauchy-Schwarz inequality A.10 and A.12 to obtain the estimate

$$
\begin{aligned}
\int_{|k|>2(d s \ell)^{-1}} \frac{\widehat{W}(k)^{2}}{|k|^{2}} \mathrm{~d} k \leq & \int_{|k|>2(d s \ell)^{-1}} \frac{\widehat{v}_{R}(k)^{2}}{|k|^{2}} \mathrm{~d} k \\
& +2\left(\int_{|k|>2(d s \ell)^{-1}} \frac{\left|\widehat{W}(k)-\widehat{v}_{R}(k)\right|^{2}}{|k|^{2}} \mathrm{~d} k\right)^{\frac{1}{2}}\left(\int_{|k|>2(d s \ell)^{-1}|k|^{2}} \mathrm{~d} k\right)^{\frac{1}{2}} \\
& +\int_{|k|>2(d s \ell)^{-1}} \frac{\widehat{v}_{R}(k)^{2}(k)-\left.\widehat{v}_{R}(k)\right|^{2}}{|k|^{2}} \mathrm{~d} k \\
\leq & \frac{1}{R} \int_{|k|>2(d s \ell)^{-1} R} \frac{\widehat{v}_{1}(k)^{2}}{|k|^{2}} \mathrm{~d} k+C|| W-v_{R}\left\|_{\frac{6}{5}}|| v_{R}\right\|_{\frac{6}{5}}+C|| W-v_{R} \|_{\frac{6}{5}}^{2} \\
\leq & \frac{1}{R} \int_{|k|>2(d s \ell)^{-1} R} \frac{\widehat{v}_{1}(k)^{2}}{|k|^{2}} \mathrm{~d} k+C\left(\frac{R}{d \ell}\right)^{2} \frac{a^{2}}{R} .
\end{aligned}
$$

Using $|\widehat{W}(k)| \leq C a$, gives

$$
\begin{aligned}
\int_{|k|>2(d s \ell)^{-1}} \widehat{W}(k)^{2}|k|^{-3} \mathrm{~d} k & =\int_{2(d s \ell)^{-1}<|k|<R^{-1}} \widehat{W}(k)^{2}|k|^{-3} \mathrm{~d} k+\int_{|k|>R^{-1}} \widehat{W}(k)^{2}|k|^{-3} \mathrm{~d} k \\
& \leq C \int_{2(d s \ell)^{-1} R<|k|<1} \widehat{W}(0)^{2}|k|^{-3} \mathrm{~d} k+R \int_{|k|>2(d s \ell)^{-1}} \widehat{W}(k)^{2}|k|^{-2} \mathrm{~d} k
\end{aligned}
$$




$$
\leq C a^{2} \ln \left(\frac{d s \ell}{R}\right)
$$

Combining (A.13), A.14 and A.15), we arrive at

$$
\begin{aligned}
\int_{|k|>2(d s \ell)^{-1}} \frac{\widehat{W}(k)^{2}}{\tau_{B}\left(k^{2}\right)} \mathrm{d} k & \leq\left(1+C \varepsilon_{0}\right) \int_{|k|>2(d s \ell)^{-1}} \frac{\widehat{W}(k)^{2}}{|k|^{2}} \mathrm{~d} k+C(d s \ell)^{-1} \int_{|k|>2(d s \ell)^{-1}} \frac{\widehat{W}(k)^{2}}{|k|^{3}} \mathrm{~d} k \\
& \leq\left(1+C \varepsilon_{0}\right) \frac{1}{R} \int \frac{\widehat{v}_{1}(k)^{2}}{|k|^{2}} \mathrm{~d} k+C a \frac{a}{d s \ell} \ln \left(\frac{d s \ell}{R}\right) .
\end{aligned}
$$

In the last inequality we used that the second term in $(A .14$ and the positive term $\left(1+C \varepsilon_{0}\right) \frac{1}{R} \int_{|k|<2(d s \ell)^{-1} R} \frac{\widehat{v}_{1}(k)^{2}}{|k|^{2}} \mathrm{~d} k$ by Condition 1 are bounded by the last term in A.16).

\section{A.1.2 Estimates on the Large Box}

Recall that $\tau_{B}\left(k^{2}\right)=\left(1-\varepsilon_{0}\right)\left(1-\varepsilon_{T}\right)\left[|k|-\frac{1}{2}(s \ell)^{-1}\right]_{+}^{2}+\left(1-\varepsilon_{0}\right) \varepsilon_{T}\left[|k|-\frac{1}{2}(d s \ell)^{-1}\right]_{+}^{2}$ and $\int \chi_{B}^{2}=|B|$ on the large box. Hence, as in A.7),

$$
\int_{|k|<(s \ell)^{-1}} h_{0}(k) \mathrm{d} k \geq-\int_{|k|<(s \ell)^{-1}} \frac{n_{0}}{|B|} \widehat{W}(k) \mathrm{d} k \int \chi_{B}^{2} \geq-C \frac{n}{|B|} a(s \ell)^{-3}|B| .
$$

Analogously to A.8 we have

$$
\frac{1}{2}(2 \pi)^{-3} \int_{|k|>(s \ell)^{-1}} h_{0}(k) \mathrm{d} k \geq \frac{1}{2}(2 \pi)^{-3} \int_{|k|>(s \ell)^{-1}}-\frac{1}{2} \frac{n^{2}}{|B|^{2}} \frac{\widehat{W}(k)^{2}}{\tau_{B}\left(k^{2}\right)} \mathrm{d} k|B|-C \int_{|k|>R^{-1}} \frac{n^{3}}{|B|^{3}} \frac{|\widehat{W}(k)|^{3}}{\tau_{B}\left(k^{2}\right)^{2}} \mathrm{~d} k|B| .
$$

The last term in A.18 is estimated in the same way as the last term in A.8

$$
\int_{|k|>R^{-1}} \frac{n^{3}}{|B|^{3}} \frac{|\widehat{W}(k)|^{3}}{\tau_{B}\left(k^{2}\right)^{2}} \mathrm{~d} k \int \chi_{B}^{2} \leq C \int_{|k|>R^{-1}} \frac{n^{3}}{|B|^{3}} \frac{a^{3}}{|k|^{4}} \mathrm{~d} k|B| \leq C \frac{n^{3}}{|B|^{3}} a^{3} R|B| .
$$

By (19) we have $v_{R}(x) \leq W(x) \leq v_{R}(x)\left(1+C\left(\frac{R}{\ell}\right)^{2}\right)$ and hence, similarly to A.14,

$$
\int_{|k|>(s \ell)^{-1}} \frac{\widehat{W}(k)^{2}}{|k|^{2}} \mathrm{~d} k \leq \int_{|k|>(s \ell)^{-1}} \frac{\widehat{v}_{R}(k)^{2}}{|k|^{2}} \mathrm{~d} k+C \frac{a^{2} R}{\ell^{2}} .
$$


Similarly to A.15 we have

$$
\int_{|k|>(s \ell)^{-1}} \widehat{W}(k)^{2}|k|^{-3} \mathrm{~d} k \leq C a^{2} \ln \left(\frac{s \ell}{R}\right)
$$

Since

$$
\tau_{B}\left(k^{2}\right)^{-1} \leq \begin{cases}\left(1+C \varepsilon_{0}+C \varepsilon_{T}\right)|k|^{-2}+C(s \ell)^{-1}|k|^{-3} & \text { for }(s \ell)^{-1}<|k|<(d s \ell)^{-1} \\ \left(1+C \varepsilon_{0}\right)|k|^{-2}+C\left((s \ell)^{-1}+\varepsilon_{T}(d s \ell)^{-1}\right)|k|^{-3} & \text { for }|k| \geq(d s \ell)^{-1}\end{cases}
$$

we have, similar to A.16,

$$
\begin{aligned}
\int_{|k|>(s \ell)^{-1}} \frac{\widehat{W}(k)^{2}}{\tau_{B}\left(k^{2}\right)} \mathrm{d} k \leq & \left(1+C \varepsilon_{0}+C \varepsilon_{T}\right) \int_{(s \ell)^{-1}<|k|<(d s \ell)^{-1}} \frac{\widehat{W}(k)^{2}}{|k|^{2}}+C(s \ell)^{-1} \frac{\widehat{W}(k)^{2}}{|k|^{3}} \mathrm{~d} k \\
& +\left(1+C \varepsilon_{0}\right) \int_{|k|>(d s \ell)^{-1}} \frac{\widehat{W}(k)^{2}}{|k|^{2}}+C\left((s \ell)^{-1}+\varepsilon_{T}(d s \ell)^{-1}\right) \frac{\widehat{W}(k)^{2}}{|k|^{3}} \mathrm{~d} k \\
\leq & \int_{|k|>(s \ell)^{-1}} \frac{\widehat{v_{R}}(k)^{2}}{|k|^{2}} \mathrm{~d} k+C \varepsilon_{0} a \frac{a}{R}+C \frac{a^{2} R}{\ell^{2}}+C \epsilon_{T} a^{2}(d s \ell)^{-1} \\
& +C(s \ell)^{-1} a^{2} \ln \left(\frac{s \ell}{R}\right)+C \varepsilon_{T}(d s \ell)^{-1} a^{2} \ln \left(\frac{d s \ell}{R}\right) \\
\leq & \int \frac{\widehat{v_{R}}(k)^{2}}{|k|^{2}} \mathrm{~d} k+C \varepsilon_{0} a \frac{a}{R}+C(s \ell)^{-1} a^{2} \ln \left(\frac{s \ell}{R}\right) \\
& +C \varepsilon_{T}(d s \ell)^{-1} a^{2} \ln \left(\frac{d s \ell}{R}\right) .
\end{aligned}
$$

In the last inequality we used that $C \frac{a R}{\ell^{2}} a$ and the positive term $\int_{|k|<(s \ell)^{-1}} \frac{\widehat{v}_{R}(k)^{2}}{|k|^{2}} \mathrm{~d} k$ by Condition 1 are bounded by the second to last term in A.23.

\section{Acknowledgments}

This project started out many years ago from a fruitful discussion with E. H. Lieb, who the authors are very grateful to. The authors acknowledge support by ERC Advanced grant 321029 and by VILLUM FONDEN via the QMATH Centre of Excellence (Grant No. 10059). 


\section{References}

[1] N. N. Bogoliubov, On the theory of superfluidity, Izv. Akad. Nauk USSR, 11, 77 (1947). Eng. Trans. J. Phys. (USSR), 11, 23 (1947). See also Lectures on quantum statistics, Gordon and Breach (1968).

[2] J. G. Conlon, E. H. Lieb and H.-T. Yau, The $N^{7 / 5}$ law for charged bosons, Commun. Math. Phys. 116, 417-448 (1988).

[3] F. J. Dyson, Ground-State Energy of a Hard-Sphere Gas, J. Math. Phys. 106, 20-26 (1957).

[4] L. Erdős, B. Schlein, and H.-T. Yau, Ground state energy of a low-density Bose gas: A second-order upper bound, Phys. Rev. A 78(5) (2008).

[5] L. L. Foldy, Charged Boson Gas, Phys. Rev. 124, 649-651 (1961); Errata ibid 125, 2208 (1962).

[6] M. Girardeau and R. Arnowitt, Theory of Many-Boson Systems: Pair Theory, Phys. Rev. 113, 755-761 (1959).

[7] A. Giuliani and R. Seiringer, The Ground State Energy of the Weakly Interacting Bose Gas at High Density, J. Stat. Phys. 135, 915-934 (2009); arXiv: 0811.1166 (2008).

[8] T. D. Lee, K. Huang and C. N. Yang, Eigenvalues and Eigenfunctions of a Bose System of Hard Spheres and its Low-Temperature Properties, Phys. Rev. 106, 1135-1145 (1957).

[9] W. Lenz, Die Wellenfunktion und Geschwindigkeitsverteilung des entarteten Gases, Zeit. f. Physik 56, 778-789 (1929).

[10] E. H. Lieb, The Bose Fluid in Lecture Notes in Theoretical Physics VIIC, edited by W. E. Brittin, Univ. of Colorado Press, 175-224 (1964).

[11] E. H. Lieb, Simplified Approach to the Ground-State Energy of an Imperfect Bose Gas, Phys. Rev. 130, 2518-2528 (1963).

[12] E. H. Lieb and W. Liniger, Exact Analysis of an Interacting Bose Gas. I. The General Solution and the Ground State, Phys. Rev. 130, 1605-1616 (1963).

[13] E. H. Lieb and J. P. Solovej, Ground State Energy of the One-Component Charged Bose Gas, Commun. Math. Phys. 217, 127-163 (2001). Ibid Erratum 225, 219-221 (2002). arXiv: cond-mat/0007425. 
[14] E. H. Lieb and J. P. Solovej, Ground State Energy of the Two-Component Charged Bose Gas, Commun. Math. Phys. 252, 485-534 (2004). arXiv: math-ph/0311010.

[15] E. H. Lieb, R. Seiringer, J. P. Solovej, and J. Yngvason, The Mathematics of the Bose Gas and its Condensation, Birkhäuser, (2005).

[16] E. H. Lieb and J. Yngvason, Ground State Energy of the Low Density Bose Gas, Phys. Rev. Lett. 80, 2504-2507 (1998).

[17] N. Napiórkowski, R. Reuvers and J. P. Solovej, The Bogoliubov Free Energy Functional II: The Dilute Limit, Commun. Math. Phys. 360, 347-403 (2017)

[18] D. W. Robinson, The Thermodynamic Pressure in Quantum Statistical Mechanics, Lecture Notes in Physics, 9, Springer, (1971).

[19] H.-T. Yau and J. Yin, The Second Order Upper Bound for the Ground Energy of a Bose Gas, J. Stat. Phys. 136, 453-503, arXiv: 0903.5347 (2009).

[20] A. Aaen, The Ground State Energy of a Dilute Bose Gas in Dimension $n>3$, arXiv: $1401.5960,(2014)$.

[21] E. H. Lieb and M. Loss, Analysis, American Mathematical Society, (2001).

[22] B. Brietzke, On the Second Order Correction to the Ground State Energy of the Dilute Bose Gas, PhD Thesis, (2017).

[23] B. Brietzke, S. Fournais, J. P. Solovej, A simple 2nd order lower bound to the energy of dilute Bose gases, eprint, (2019).

[24] C. Boccato, C. Brennecke, S. Cenatiempo, B. Schlein, Bogoliubov Theory in the GrossPitaevskii Limit, arXiv: 1801.01389, (2018).

[25] C. Boccato, C. Brennecke, S. Cenatiempo, B. Schlein, Complete Bose-Einstein Condensation in the Gross-Pitaevskii regime, Commun. Math. Phys. 359, 975-1026 (2018), arXiv: 1703.04452 (2017).

[26] C. Boccato, C. Brennecke, S. Cenatiempo, B. Schlein, Optimal Rate for Bose-Einstein Condensation in the Gross-Pitaevskii Regime, arXiv: 1812.03086 (2018).

[27] C. Boccato, C. Brennecke, S. Cenatiempo, B. Schlein, The excitation spectrum of Bose gases interacting through singular potentials, arXiv: 1704.04819 (2017). 